\title{
Extensōes da Teoria das Equações de Estimação Generalizadas a Dados Circulares e Modelos de Dispersão
}

\author{
Rinaldo Artes \\ TESE APRESENTADA \\ $\mathrm{AO}$ \\ INSTITUTO DE MATEMÁTICA E ESTATÍSTICA \\ DA \\ UNIVERSIDADE DE SÃO PAULO \\ PARA OBTENÇÃO DO GRAU DE DOUTOR \\ EM ESTATÍSTICA \\ ORIENTADOR: PROF. DR. GILBERTO ALVARENGA PAULA \\ SÃO PAULO. ABRIL DE 1997
}




\title{
Extensões da Teoria das Equações de Estimação Generalizadas a Dados Circulares e Modelos de Dispersão
}

\begin{abstract}
Este exemplar corresponde à redação final da tese devidamente corrigida, defendida por Rinaldo Artes e aprovada pela Comissão Julgadora.
\end{abstract}

São Paulo, abril de 1997.

Banca Examinadora

Prof. Dr. Gilberto Alvarenga Paula (IME-USP)

Prof. Dr. Heleno Bolfarine (IME-USP)

Prof. Dr. Gauss Moutinho Cordeiro (UFPE)

Prof. Dr. Ruben Klein (LNCC-RJ)

Profa. Dra. Eliana Heiser de F. Marques (IMECC-UNICAMP) 
Amélia,

Luiza

e

Marcelo 


\section{AGRADECIMENTOS INSTITUCIONAIS}

Parte da pesquisa apresentada neste tese foi desenvolvida no Departamento de Estatística da Universidade de British Columbia, em Vancouver, Canadá. Esses estudos foram parcialmente financiados pelo CN Pq através de uma Bolsa Sanduíche no Exterior, processo 201190-94.0.

Os estudos de simulação apresentados no Capítulo 5 desta tese foram parcialmente realizados utilizando-se recursos do Laboratório de Computação Científica e Avançada, instalado no Centro de Computação Eletrônica da USP.

$\mathrm{O}$ candidato e o orientador fazem parte do projeto temático: Métodos Assintóticos em Regressão, código 96/01741-7, da FAPESP. 


\section{AGRADECIMENTOS}

Ao

Prof.Dr. Gilberto Alvarenga Paula, pelo entusiasmo transmitido durante a orientação, pela sugestão do tema e pelo incentivo e apoio durante todas as etapas da elaboração deste trabalho.

Ao

Prof.Dr. Bent Jørgensen, pela orientação, disponibilidade e pela oportunidade de desenvolver boa parte deste trabalho junto ao Departamento de Estatística da Universidade de British Columbia (UBC), ao qual agradeço, bem como pela cordial recepção.

Ao

Prof.Dr. Pablo Ferrari, pelo apoio que possibilitou meu estágio na UBC.

Ao

Prof. Dr. José Galvão Leite, pelas discussões sobre alguns resultados desta tese.

Ao

Prof. Dr. Ronald Ranvaud, por ter fornecido os dados analisados nesta tese.

À

Profa. Lisbeth K. Cordani, que se sujeitou a ler este trabalho, pelas sugestões e incentivo durante a elaboração da tese.

A

Antonio Carlos P. Lima, Elisabeti Kira, Marcia Branco, Loretta Gasco Campos e Thelma Safadi, pelo apoio.

E, finalmente, a Amélia, Luiza e Marcelo pela companhia, carinho e incentivo, que tornaram mais agradável o período de desenvolvimento deste estudo. 


\section{Resumo}

Nesta tese sugerimos um método inspirado na teoria da quase-verossimilhança para a análise de modelos de dispersão longitudinais. Tal método não pressupõe o conhecimento da distribuição multivariada associada aos vetores resposta, necessitando apenas de suposições sobre o comportamento dos parâmetros de interesse e sobre a estrutura de correlação envolvendo funções convenientes dos dados. Foram desenvolvidas equaçôes de estimação tanto para modelos envolvendo o parämetro de posição como para modelos que envolvem tanto o parâmetro de posição como o de dispersão. Provamos, sob condições gerais, que os estimadores obtidos por esse método são consistentes e assintoticamente normais. Outras propriedades desses estimadores foram avaliadas através de estudos de simulação. Apresentamos, também, uma aplicação à um conjunto de dados circulares. 


\begin{abstract}
In this thesis we introduced a method based on the quasi-likelihood theory for the analysis of longitudinal dispersion models. The method does not require the knowledge of the multivariate distribution of the response vectors. It is necessary some asumptions concerning the behaviour of the parameters of interest and of the correlation structure of some data functions. We developd estimating equations for position and dispersion parameters that are generalizations of generalized estimating equations of Liang and Zeger. We also proved that, under general regularity conditions, the estimators obtained by this method are consistent and asymptotically normal. Further properties were evaluated by simulation studies. The method was applied to a real data set of circular variables.
\end{abstract}




\section{Sumário}

1 Introdução 4

1.1 Dados circulares. . . . . . . . . . . . . . . . . 5

1.1.1 Representação gráfica e conceitos básicos . . . . . . 6

1.1.2 Modelos probabilísticos . . . . . . . . . . . . 8

1.1.3 Relações entre as distribuições uniforme circular, von Mises e normal arqueada . . . . . . . . . . . . . 11

1.1.4 Modelos de regressão . . . . . . . . . . . . . . . . 11

1.1.5 Dependência entre variáveis circulares . . . . . . . 13

1.1.6 Modelos de séries temporais para variáveis circulares . 15

1.2 Modelos de dispersão . . . . . . . . . . . . . . . . . . 16

1.2.1 Exemplos de modelos de dispersão . . . . . . . . . 18

1.2.2 Inferências para um modelo de dispersão . . . . . . . 20

1.3 Apresentação dos capítulos . . . . . . . . . . . . . . . . . 23

2 Funções de estimação 24

2.1 Função de estimação ótima . . . . . . . . . . . . . . 27

2.2 Alguns resultados assintóticos . . . . . . . . . . . . . . 29

2.2 .1 Complemento . . . . . . . . . . . . . . . . . 33

2.2 .2 Aplicação . . . . . . . . . . . . . . . . . . 34

2.2 .3 Demonstrações . . . . . . . . . . . . . . . 37

3 Funções de estimação para modelos de dispersão longitudinais 43

3.1 Modelagem do parâmetro de posição . . . . . . . . . . . 44

3.1.1 $\operatorname{Cov}\left(\mathbf{u}_{i}\right)$ conhecida . . . . . . . . . . 45

$3.1 .2 \operatorname{Cov}\left(\mathbf{u}_{i}\right)$ desconhecida . . . . . . . . . . . 46

3.1 .3 Estimação de $\sigma^{2} \ldots \ldots \ldots \ldots$. . . . . . . . . . . 48

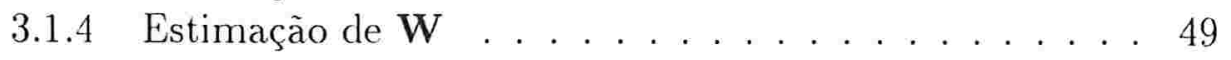


3.1.5 Teste de hipóteses . . . . . . . . . . . . . . . 53

3.1.6 Algoritmos de estimação . . . . . . . . . . . . . . . 53

3.1.7 Demonstrações . . . . . . . . . . . . . . . . . . . 54

3.2 Exemplos . . . . . . . . . . . . . . . . . 57

3.2.1 Aplicação a modelos exponenciais de dispersão . . . . . 57

3.2 .2 Aplicação a dados circulares . . . . . . . . . . . . . 61

3.3 Modelo misto . . . . . . . . . . . . . . . . 63

3.3.1 Algoritmos de estimação . . . . . . . . . . . . . . . . 67

3.3.2 Aplicação a dados circulares . . . . . . . . . . . . 68

4 Normal arqueada multivariada e simulações $\quad 73$

4.1 Distribuição normal arqueada multivariada . . . . . . . . . . . 73

4.1.1 Modelos para a análise de dados longitudinais . . . . . 75

4.1.2 Algoritmos de estimação . . . . . . . . . . . . . 75

4.1.3 Estimação dos parâmetros de regressão via funções de estimação . . . . . . . . . . . 76

4.2 Simulações . . . . . . . . . . . . . . . . 76

4.2.1 Geração da normal arqueada multivariada . . . . . . . 77

4.2 .2 Estudo $1 \ldots \ldots \ldots \ldots$. . . . . . . . . . . 77

4.2 .3 Estudo 2 . . . . . . . . . . . . . . . . . . . . . . 81

4.2 .4 Conclusões . . . . . . . . . . . . . . . . 85

5 Aplicação $\quad 86$

5.1 O conjunto de dados . . . . . . . . . . . . . 86

5.2 Modelagem do problema . . . . . . . . . . . . . 88

5.2 .1 Suposições . . . . . . . . . . . . . . . . . . . . 88

5.2 .2 Modelo. . . . . . . . . . . . . . . . . 90

5.3 Procedimento de estimação dos modelos . . . . . . . . . . . . 91

5.4 Análise dos dados . . . . . . . . . . . . . . . . . . . . . . . . . 93

5.4.1 Conclusão da análise . . . . . . . . . . . . . . 94

5.5 Comparações . . . . . . . . . . . . . . . . . . . . . . . 98

5.5.1 Conclusòes . . . . . . . . . . . . . . . . . . 101

5.6 Detalhes técnicos . . . . . . . . . . . . . . . . 101

6 Conclusões e futuros estudos 103

6.1 Futuros estudos . . . . . . . . . . . . . . . . . . . . . 104

6.1.1 Modelos de séries temporais com covariáveis . . . . . . 104

6.2 Geração de distribuições circulares multivariadas . . . . . . . . 107 
6.2.1 Estimação da matriz de covariâncias dos parámetros obtidos a partir das funções de estimação tipo "EEG" . 110

Referências Bibliográficas

111

\section{APÊNDICES}

$\begin{array}{lr}\text { A Funções de Bessel } & 118\end{array}$

A.1 Derivadas de $I_{0}, I_{1}$ e $\mathcal{A}_{1} \ldots \ldots \ldots \ldots$. . . . . . . . . . . . . . . . . . . . . . . . . .

A.2 Cálculo das funções . . . . . . . . . . . . . . . . . . . . . . . . 119

A.2.1 Fórmulas de recorrência . . . . . . . . . . . . . 119

A.2.2 Avaliação de $I_{0}$ e $I_{1} \ldots \ldots \ldots$. . . . . . . . . . . . 119

A.2.3 Avaliação da função inversa de $\mathcal{A}_{1} \ldots$. . . . . . . . 120

B Resultados adicionais relativos a dados circulares $\quad 121$

B.1 Intervalos de confiança . . . . . . . . . . . . . . . . 121

B.2 Outros resultados . . . . . . . . . . . . . . . . . . 122

$\begin{array}{ll}\text { C Resultados adicionais } & 123\end{array}$

D Avaliação de erros para o cálculo da densidade da normal arqueada 124

D.1 Avaliação das séries via truncamento . . . . . . . . . . . . 124

$\begin{array}{ll}\text { E Complemento da aplicação } & 127\end{array}$

$\begin{array}{lll}\text { F Resultados relativos a cópulas } & 129\end{array}$ 


\section{Capítulo 1}

\section{Introdução}

$\mathrm{Na}$ literatura Estatística, existe uma grande variedade de técnicas adequadas ao tratamento de dados longitudinais quando a variável resposta segue uma distribuição Gaussiana. O fato de existir uma versão multivariada da distribuição normal com boas propriedades do ponto de vista inferencial, facilita o estudo desse caso. Abandonando-se a suposição de normalidade da variável resposta, uma série de dificuldades pode surgir devido à escassez de distribuições multivariadas alternativas com tais propriedades (ver comentário em Zeger e Liang, 1986).

Dentre as várias alternativas de tratamentos para dados longitudinais, duas assumem um papel de destaque. A primeira prevê a modelagem probabilística do problema, estipulando a priori uma distribuição multivariada de probabilidades, supostamente adequada à modelagem dos dados e, então, com base nessa distribuição, sugerindo um estimador para os seus parâmetros (por exemplo, através do método de máxima verossimilhança). As dificuldades dessa abordagem estão ligadas à definição do modelo probabilístico, ou seja, em como gerar um modelo multivariado que se ajuste aos dados e tenha parâmetros facilmente estimáveis. A segunda possibilidade baseia-se no uso de funções de estimação (ver Godambe, 1991, por exemplo) para a obtenção da estimativa de alguns parâmetros de um modelo multivariado que não é, necessariamente, completamente conhecido. De maneira simplificada, podese afirmar que uma função de estimação é uma função dos parâmetros de interesse e dos dados. Em termos práticos, ela é construída de tal modo que, quando é vista como função dos parâmetros, suas raízes são estimativas dos mesmos. A equação obtida ao se igualar uma função de estimação a zero é denominada equação de estimação. Nessa abordagem, troca-se a exigência de 
se conhecer um modelo probabilístico completo por suposições teoricamente mais simples, por exemplo, algum conhecimento a priori sobre a estrutura de correlação dos dados e sobre o comportamento da média da distribuição. A teoria da quase-verossimilhança de Wedderburn (1974) é um exemplo de desenvolvimento teórico que vai nessa linha de métodos. A dificuldade está na construção de equações de estimação que levem a estimativas que tenham algumas propriedades interessantes, pelo menos do ponto de vista assintótico.

O objetivo original deste trabalho é a introdução de novas técnicas de estimação que possibilitem a análise de dados circulares longitudinais. Com esse fim, desenvolvemos equações de estimação aplicáveis a uma classe ampla de modelos (modelos de dispersão) que levam a estimativas consistentes e assintoticamente normais de parâmetros de regressão de um modelo longitudinal. Nosso método generaliza o método das equações de estimação generalizadas proposto por Liang e Zeger (1986), para análise de dados com marginais na família exponencial.

\subsection{Dados circulares}

Seja $z$ uma variável aleatória absolutamente contínua definida no espaço amostral $(\Re, \mathcal{A})$, onde $\mathcal{A}$ representa os borelianos de $\Re$. Uma variável aleatória circular contínua $y$ pode ser definida como $y=a+\{z(\bmod m)\}$, onde $a \in \Re, m>0$ e o operador mod está indicando o resto da divisão de $z$ por $m$. Um exemplo importante é o caso em que $y$ corresponde a um ângulo (variável angular). Nessa situação todos os valores $y+2 k \pi, k=\ldots,-2,-1,0,1,2, \ldots$ correspondem ao mesmo ângulo.

Uma variável quantitativa absolutamente contínua circular difere de uma não-circular (doravante denominada linear), por possuir uma métrica bastante singular. Para ilustrar essa particularidade, suponha que se tenha observado dois ângulos: o primeiro sendo $15^{\circ}$ e o segundo $35^{\circ}$. Pode-se questionar sobre qual seria o valor do ângulo médio dessas observaçòes. Ao se calcular a média aritmética chega-se a $25^{\circ}$, o que parece ser bastante razoável. Suponha agora que a origem dos ângulos seja modificada através da aplicação de uma rotação de $-25^{\circ}$. Os valores transformados passam a ser $350^{\circ}$ e $10^{\circ}$, que têm $180^{\circ}$ como média aritmética. Esse valor está muito distante dos observados. Baseando-se nos dados, pode-se intuir que o valor "médio" deveria ser $0^{0}$ 1. Na verdade, a distância entre duas observaçôes de uma variável circular,

\footnotetext{
${ }^{1}$ valor que corresponde à denominada média circular dos dados, cujo conceito será
} 
$a<b$, é dada por $\min \{|a-b|(\bmod m) ;|a+m-b|(\bmod m)\}$.

$\mathrm{O}$ estudo de dados circulares é bastante antigo e tem sua origem no estudo da posição de corpos celestes (ver Bernoulli, 1734, por exemplo). Como exemplos de variáveis circulares temos: a direção tomada por animais após certo tratamento, a direção do vento; a direção de correntes marítimicas, o horário de falha de um sistema, o horário de entrada de pacientes em uma UTI, etc. Fisher (1993) apresenta 24 conjuntos de dados circulares reais.

O procedimento padrão na análise de dados com essa natureza é convertè-los em ângulos, através de uma transformação linear e analisá-los nessa escala. A partir de agora, não se fará distinção entre os termos "circular" e "angular" e, a não ser que seja explicitado, considerar-se-á a variável já convertida em ângulos. Em Mardia (1972), Batschelet (1981) e Fisher (1993), encontra-se uma grande variedade de técnicas e modelos probabilísticos para dados circulares.

\subsubsection{Representação gráfica e conceitos básicos}

Usualmente, uma observação $y$ de uma variável circular pode ser representada graficamente em um círculo de raio unitário na posição $(1, y)$, quando se utiliza coordenadas polares, ou $(\cos (y)$, $\operatorname{sen}(y))$, quando se utiliza coordenadas cartesianas. A Figura 1.1 é uma representação gráfica de uma observação $y$, de uma variável circular.

A média circular de um conjunto de dados circulares, $y_{i}, i=1,2, \ldots, n$, é dada por

$$
\hat{\mu}= \begin{cases}\arctan (S / C), & \text { se } S \geq 0 \text { e } C>0 \\ \arctan (S / C)+\pi, & \text { se } C<0 \\ \arctan (S / C)+2 \pi, & \text { se } S<0 \text { e } C>0\end{cases}
$$

onde $S==\sum_{i=1}^{n} \operatorname{sen}\left(y_{i}\right)$ e $C^{\prime}=\sum_{i=1}^{n} \cos \left(y_{i}\right)$. Essa medida corresponde ao centro de gravidade dos dados. Outra interpretação possível pode ser obtida quando se considera cada observação como um vetor de comprimento um e direção $y_{i}$. Nesse caso, a média circular corresponde à direção do vetor resultante.

Uma medida de concentração bastante utilizada na análise de dados circulares é o comprimento do vetor resultante,

$$
R=\sqrt{S^{2}+C^{2}} \text {. }
$$

apresentado na seção 1.1.1 


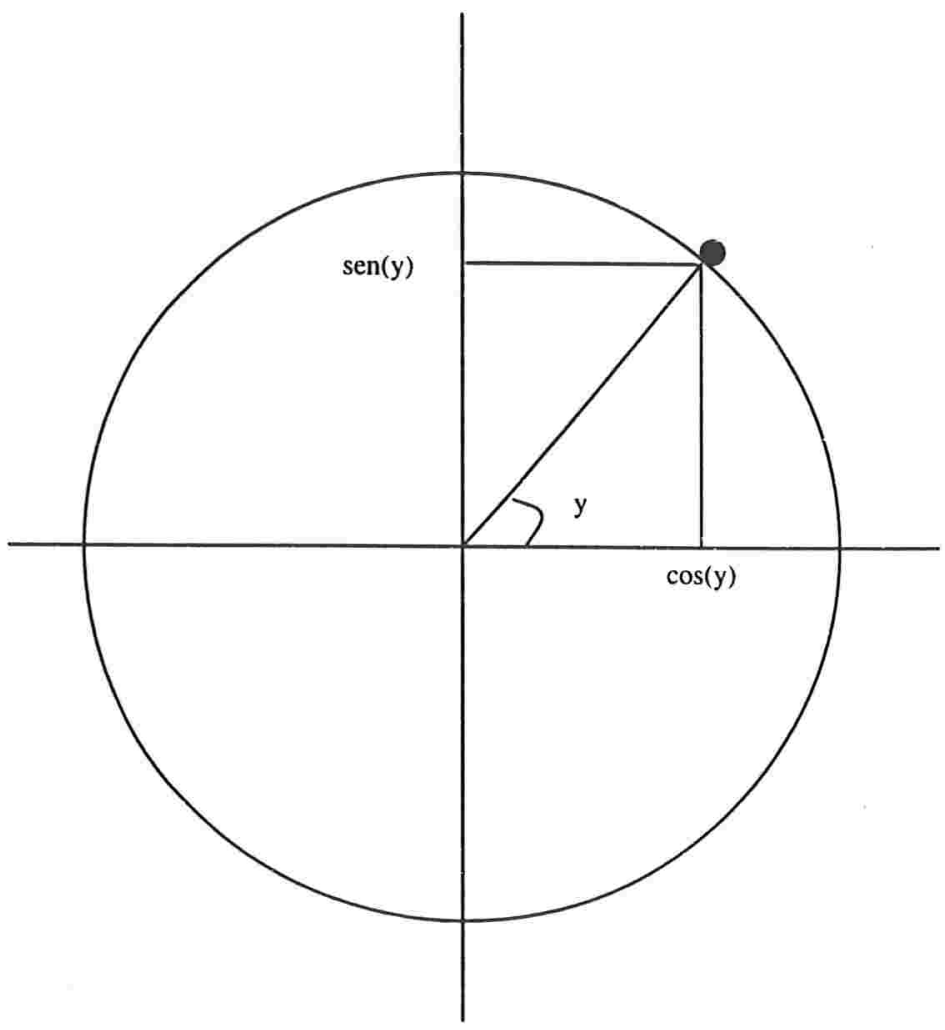

Figura 1.1: Representação gráfica de uma observaçào circular 

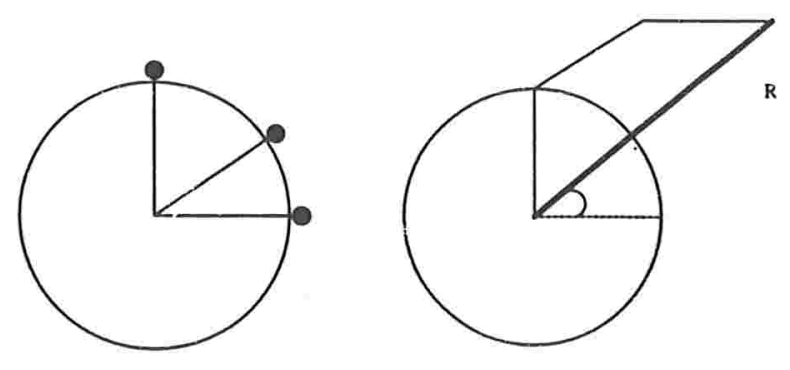

Figura 1.2: Representação gráfica de três observações circulares e de sua correspondente média circular e comprimento da resultante

No caso de todas as observações serem coincidentes, tem-se que $R=n$; esse é o caso de concentração máxima dos dados (variabilidade mínima). Outro caso limite se dá quando os ângulos encontram-se uniformemente distribuídos no círculo, onde, $R=0$; trata-se do caso de concentração mínima (variabilidade máxima). Note que o conceito de variabilidade máxima não existe para dados lineares. Cabe salientar que quando a variabilidade é máxima, a média circular não está definida. Usualmente utiliza-se o comprimento da resultante média, definido por

$$
\bar{R}=\frac{R}{n},
$$

que tem a vantagem de variar no intervalo $[0,1]$.

A Figura 1.2 traz a representação gráfica da resultante e da média circular (ângulo indicado na figura) calculadas para um conjunto de 3 observações.

\subsubsection{Modelos probabilísticos}

Represente por $p$ e $F$, respectivamente, a função densidade de probabilidades e a função distribuição de uma variável aleatória circular $y$. Admita $y \in S_{2 \pi}$, onde $S_{2 \pi}$ é um intervalo semi-aberto de amplitude $2 \pi$. Define-se a função característica circular (ver Mardia, 1972, por exemplo) de $y$ no ponto $r=$ $1,2, \ldots$ como $\phi_{r}=\alpha_{1 r}+i \alpha_{2 r}, \operatorname{com} \alpha_{1 r}=E\{\cos (r y)\}$ e $\alpha_{2 r}=E\{\operatorname{sen}(r y)\}$. $\alpha_{1 r}$ e $\alpha_{2 r}$ são denominados momentos circulares de $y$. Para $r=1$, tem-se

$$
\phi_{1}=\rho e^{i \mu}
$$

onde $\mu$ é a média circular e $\rho$ é o comprimento da resultante média de y. 
Mardia (1972) mostra que

$$
p(y)=\frac{1}{2 \pi}\left[1+2 \sum_{r=1}^{\infty}\left\{\alpha_{1 r} \cos (r y)+\alpha_{2 r} \operatorname{sen}(r y)\right\}\right]
$$

e

$$
F(y)=\frac{1}{2 \pi}\left[y+2 \sum_{r=1}^{\infty} \frac{\alpha_{1 r} \operatorname{sen}(r y)+\alpha_{2 r} \cos (r y)}{r}\right] .
$$

Dentre os vários modelos probabilísticos adequados à análise de dados circulares, três serão destacados: as distribuições uniforme circular, von Mises e normal arqueada (tradução livre do termo "wrapped normal"). Outros modelos podem ser encontrados em Mardia (1972), Watson (1983) e Fisher (1993).

\section{Distribuição uniforme circular}

A distribuição uniforme circular possui função densidade de probabilidades dada por

$$
p(y)=\frac{1}{2 \pi}, y \in S_{2 \pi} .
$$

Uma característica que merece destaque é o fato dessa distribuição não possuir média circular e ter comprimento da resultante média igual a zero. Esse fato é bastante importante uma vez que a distribuição uniforme circular tem um papel central na teoria assintótica desenvolvida para dados circulares (ver Mardia, 1972, por exemplo).

\section{Distribuição von Mises}

A distribuição von Mises é uma das mais utilizadas na modelagem de dados reais. Isso se deve ao fato de sua densidade ter uma forma relativamente simples, possuir boas propriedades (regularidade, parâmetros interpretáveis e simetria) e parâmetros que são facilmente estimáveis (veja os estimadores no Apêndice B). Se $y \in S_{2 \pi}$ segue uma distribuição von Mises. com média circular $\mu \in S_{2 \pi}$ e parâmetro de concentração $\lambda>0$, representa-se $y \sim$ $\mathcal{M}(\mu, \lambda)$, sua função densidade de probabilidades é dada por

$$
p(y ; \mu, \lambda)=\frac{1}{2 \pi I_{0}(\lambda)} \exp \{\lambda \cos (y-\mu)\}
$$

onde $I_{0}(\lambda)$ é a função modificada de Bessel de primeiro tipo e ordem zero, avaliada no ponto $\lambda$ (ver Abramowitz e Stegun, 1970, por exemplo). De um 
modo geral, tem-se

$$
I_{r}(\lambda)=\sum_{k=0}^{\infty}\{(k+r) ! k !\}^{-1}\left(\frac{1}{2} \lambda\right)^{2 k+r}, r=0,1,2, \ldots
$$

Os momentos trigonométricos de uina distribuição von Mises com parâmetros $\mu$ e $\lambda$ são

$$
E\{\cos (r y)\}=\mathcal{A}_{r}(\lambda) \cos (r \mu) \text { e } E\{\operatorname{sen}(r y)\}=\mathcal{A}_{r}(\lambda) \operatorname{sen}(r \mu),
$$

onde $\mathcal{A}_{r}(\lambda)=I_{r}(\lambda) / I_{0}(\lambda)$.

$\mathrm{O}$ comprimento da resultante média é dado por $\mathcal{A}_{1}(\lambda)$.

No Apêndice A são apresentadas algumas propriedades das funções $I_{r}$ e $\mathcal{A}_{r}$ e no $\mathrm{B}$ alguns resultados adicionaitu

\section{Distribuição normal arqueada}

Seja $z \sim \mathcal{N}\left(\mu, \omega^{2}\right)$. A variável aleatória definida por $y=z(\bmod 2 \pi)$ é dita seguir uma distribuição normal arqueada com parâmetros $\mu$ e $\omega^{2}$; representase por $y \sim \mathcal{N} \mathcal{E}\left(\mu, \omega^{2}\right)$. Sua função densidade de probabilidades é dada por

$$
p\left(y ; \mu, \omega^{2}\right)=\sum_{k=-\infty}^{\infty} \frac{1}{\sqrt{2 \pi} \omega} \exp \left\{-\frac{(y+2 k \pi-\mu)^{2}}{2 \omega^{2}}\right\} ; \quad y \in S_{2 \pi} .
$$

Pode-se provar, levando-se em conta Mardia(1972, pg. 53), que os momentos circulares dessa distribuição são dados por

$$
\begin{aligned}
& E\{\cos (r y)\}=\exp \left\{-\frac{1}{2} r^{2} \omega^{2}\right\} \cos (r \mu) \mathrm{e} \\
& E\{\operatorname{sen}(r y)\}=\exp \left\{-\frac{1}{2} r^{2} \omega^{2}\right\} \operatorname{sen}(r \mu) .
\end{aligned}
$$

A média circular de $y$ é dada por $\mu(\bmod 2 \pi)$ e o comprimento da resultante média é dado por $\exp \left\{-\frac{1}{2} \omega^{2}\right\}$ (ver definições em Mardia, 1972 e Fisher, 1993). 


\subsubsection{Relações entre as distribuições uniforme circular, von Mises e normal arqueada}

A distribuição normal arqueada assemelha-se bastante a uma distribuição von Mises (ver Mardia, 1972, pgs. 66 e 67; Fisher, 1993, pg. 55; Breckling, 1989, pgs. 138-140 e Collet e Lewis, 1981). Verifica-se que se $y$ segue uma distribuição von Mises, com média circular 0 e parâmetro de concentração $\lambda$, sua distribuição pode ser aproximada por uma distribuição normal arqueada com média circular 0 e parâmetro de dispersão $\omega^{2}$, tal que

$$
\mathcal{A}_{1}(\lambda)=\exp \left(-\frac{1}{2} \omega^{2}\right)
$$

Note que essa identidade surge ao igualar-se as funções características em $p=1$, das duas distribuições.

Sejam $F$ e $G$ as funções distribuição, respectivamerte, de uma von Mises e de uma normal arqueada, com mesma média circular e satisfazendo (1.4). Stephens (1963) mostra que $\sup _{x \in[0,2 \pi[}|F(x)-G(x)|$ nunca excederá 0.0125 e que esse limite será atingido para $\lambda=1.4$. Baseado nisso, Watson (1982) sugere que a escolha entre a von Mises e a normal arqueada para a modelagem de dados circulares deva ser feita de acordo com a conveniência do analista.

A distribuição uniforme circular surge naturalmente quando $\lambda$ se aproxima. de zero, no caso de uma distribuição von Mises e quando $\omega^{2}$ tende a infinito, no caso da normal arqueada. Como a distribuição uniforme circular não possui média circular e tem comprimento da resultante média zero, tem-se que, quando $\lambda$ for muito baixo ( $\omega^{2}$ alto), as técnicas baseadas na função densidade de probabilidades (estimação por máxima verossimilhança, teste da razão de verossimilhança, etc) não produzem bons resultados. Regras empíricas que estipulam valores de $\lambda$ para os quais essas técnicas levam vantagem sobre procedimentos não paramétricos são propostas em Fisher (1993) $(\lambda>2$, na maioria das vezes ou mesmo $\lambda>1$, em alguns casos).

\subsubsection{Modelos de regressão}

Há na literatura algumas sugestões de modelos de regressão para dados circulares (ver Gould, 1969; Johnson e Wehrly, 1978 e Rivest, 1989); no entanto, apresentar-se-á, por sua generalidade, o modelo sugerido por Fisher e Lee (1992). Considere uma amostra aleatória $\left(y_{i}, \mathbf{x}_{i}\right), i=1,2, \ldots, n$, onde 
$y_{i} \sim \mathcal{M}\left(\mu_{i}, \lambda\right)$ e $\mathrm{x}_{i}$ é um vetor $p$-variado de covariáveis fixas. Seja

$$
\mu_{i}=\alpha+h\left(\eta_{i}\right), \eta_{i}=\mathbf{x}_{i}^{\top} \boldsymbol{\beta}
$$

onde $\boldsymbol{\beta}$ é um vetor paramétrico, $h(\cdot)$ é uma função $h: \Re \rightarrow[-\pi, \pi[$, duplamente diferenciável e inversível, com $h(0)=0$ e $\alpha$ é um intercepto. Uma possível escolha para $h$ é $h(a)=2 \arctan (a)$.

A função escore associada aos parâmetros desse modelo conduz às seguintes equações:

$$
\begin{gathered}
\mathbf{X}^{\top} \mathbf{H u}=\mathbf{0}, \\
\bar{R} \operatorname{sen}(\hat{\alpha})=0, \\
\bar{R} \cos (\hat{\alpha})=0, \\
\text { e } \mathcal{A}_{1}(\hat{\lambda})=\bar{R},
\end{gathered}
$$

onde $\mathbf{u}=\left(u_{i}, u_{2}, \ldots, u_{n}\right)^{\top}, u_{i}=\operatorname{sen}\left\{y_{i}-\hat{\alpha}-h\left(\mathbf{x}_{i}^{\top} \hat{\boldsymbol{\beta}}\right)\right\}, \mathbf{X}=\left(\mathbf{x}_{1}, \ldots, \mathbf{x}_{n}\right)^{\top}$, $\mathbf{H}=\operatorname{diag}\left\{\frac{\partial h}{\partial \eta_{1}}\left(\eta_{1}\right), \ldots, \frac{\partial h}{\partial \eta_{n}}\left(\eta_{n}\right)\right\}$ e $\bar{R}=(1 / n)\left(S^{2}+C^{2}\right)^{1 / 2}$, sendo

$$
\begin{aligned}
& S=\sum_{i=1}^{n} \operatorname{sen}\left\{y_{i}-\hat{\alpha}-h\left(\mathbf{x}_{i}^{\top} \hat{\boldsymbol{\beta}}\right)\right\} \mathrm{e} \\
& C=\sum_{i=1}^{n} \cos \left\{y_{i}-\hat{\alpha}-h\left(\mathbf{x}_{i}^{\top} \hat{\boldsymbol{\beta}}\right)\right\} .
\end{aligned}
$$

Em alguns casos, pode-se estar interessado na modelagem do parâmetro de dispersão. Uma situação real onde isso é importante, é o estudo da direção tomada por animais após algum tratamento, ou durante o processo migratório; a variabilidade dessa direção, tende a diminuir com o passar do tempo. Em geral, a direção é representada por um ângulo que usualmente se supõe seguir uma distribuição von Mises. No caso de independência entre as observações há opções para a modelagem do parâmetro de concentração $\lambda$ (ver Fisher, 1993 e suas referências). Considere, por exemplo, $y_{i} \sim \mathcal{M}\left(\mu_{i}, \lambda_{i}\right)$ e suponha a modelagem de $\mu_{i}$ proposta anteriormente. Fisher e Lee (1992) propõem o seguinte modelo para a modelagem do parâmetro de concentração:

$$
q\left(\lambda_{i}\right)=\mathbf{x}_{i}^{\top} \gamma
$$

onde $q(\cdot)$ é uma função de ligação positiva, por exemplo a logarítmica e $\gamma$ é um vetor de parâmetros desconhecidos. 

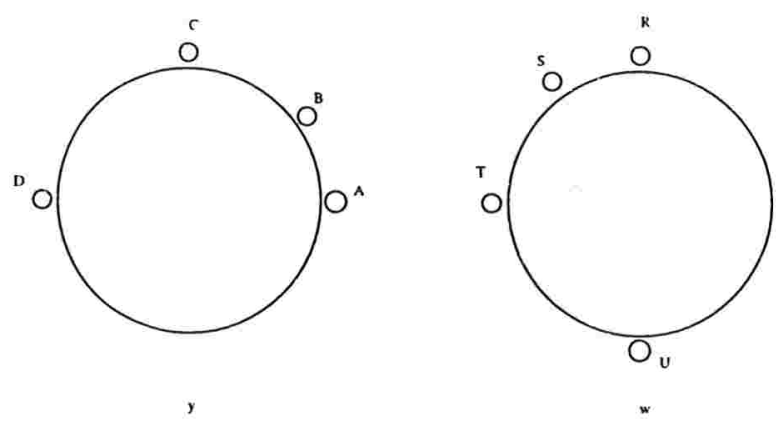

Figura 1.3: Representação gráfica de T-associação

\subsubsection{Dependência entre variáveis circulares}

Nesta seção descrevemos uma forma simples de associação entre variáveis circulares (T-associação) e introduzimos um coeficiente de correlação circular criado para medí-la (ver Fisher e Lee, 1983, para maiores detalhes). Breckling (1989) faz uma extensiva revisão sobre coeficientes de correlação entre variáveis circulares disponíveis na literatura.

Sejam $y$ e $w$ duas variáveis circulares. Diz-se que elas possuem uma associação toroidal (T-associação) positiva perfeita se

$$
y=w+\theta_{0}(\bmod 2 \pi),
$$

onde $\theta_{0}$ é um ângulo constante. Analogamente, diz-se que as variáveis possuem uma associação T-linear negativa perfeita quando

$$
y=-w+\theta_{0}(\bmod 2 \pi) .
$$

Considere as quatro observações indicadas na Figura 1.3. Note que o gráfico correspondente à variável $w$ é uma rotação do gráfico correspondente à variável $y$. Assumindo que as observaçōes sejam (A,R). (B.S), (C,T) e (D,U), tem-se uma caso de associação T-toroidal positiva perfeita.

Baseados nessas definições, Fisher e Lee (1983) desenvolvem um coeficiente de correlação para medir a intensidade dessa associação. Tome $\left(y_{1}, w_{1}\right)$ e $\left(y_{2}, w_{2}\right)$ independentes e com a mesma distribuição de $(y, w)$. Define-se o coeficiente de correlação toroidal como

$$
\rho_{T}=\frac{E\left\{\operatorname{sen}\left(y_{1}-y_{2}\right) \operatorname{sen}\left(w_{1}-w_{2}\right)\right\}}{\left[E\left\{\operatorname{sen}^{2}\left(y_{1}-y_{2}\right)\right\} E\left\{\operatorname{sen}^{2}\left(w_{1}-w_{2}\right)\right\}\right]^{1 / 2}} .
$$


Esse coeficiente possui as seguintes propriedades:

i. $-1 \leq \rho_{T} \leq 1$;

ii. $\rho_{T}=1\left(\rho_{T}=-1\right)$ se e somente se a associação toroidal entre $y$ e $w$ for perfeita positiva (negativa);

iii. $\rho_{T}$ é invariante quanto a rotações dos dados;

iv. se $y$ e $w$ são independentes, então $\rho_{T}=0 \mathrm{e}$

v. se $y$ e $w$ forem unimodais com alta concentração, então $\rho_{T}$ aproxima-se do coeficiente de correlação de Pearson.

Seja $\left(y_{i}, w_{i}\right), i=1,2, \ldots, n$, uma amostra aleatória de $n$ realizações das variáveis circulares $(y, w)$. Então, o estimador sugerido para $\rho_{T}$ é

$$
\hat{\rho}_{T}=\frac{\sum_{1 \leq i<j \leq n} \operatorname{sen}\left(y_{i}-y_{j}\right) \operatorname{sen}\left(w_{i}-w_{j}\right)}{\left\{\sum_{1 \leq i<j \leq n} \operatorname{sen}^{2}\left(y_{i}-y_{j}\right) \sum_{1 \leq i<j \leq n} \operatorname{sen}^{2}\left(w_{i}-w_{j}\right)\right\}^{1 / 2}} .
$$

Fisher e Lee (1983) demonstram que se as médias circulares de $y$ e $w$ forem iguais a zero então $n \hat{\rho}_{T}$ converge em distribuição para uma distribuição dupla exponencial com densidade $(1 / 2) \exp (-|x|)$, quando $n$ tende a infinito.

Segundo Fisher (1993), para facilitar os cálculos, a expressão (1.9) pode ser reescrita como

$$
\hat{\rho}_{T}=\frac{4(A B-C D)}{\left\{\left(n^{2}-E^{2}-F^{2}\right)\left(n^{2}-G^{2}-H^{2}\right)\right\}^{2}},
$$

onde $A=\sum_{i=1}^{n} \cos \left(y_{i}\right) \cos \left(w_{i}\right), B=\sum_{i=1}^{n} \operatorname{sen}\left(y_{i}\right) \operatorname{sen}\left(w_{i}\right), C=\sum_{i=1}^{n} \cos \left(y_{i}\right) \operatorname{sen}\left(w_{i}\right)$, $D=\sum_{i=1}^{n} \operatorname{sen}\left(y_{i}\right) \cos \left(w_{i}\right), E=\sum_{i=1}^{n} \cos \left(2 y_{i}\right), F=\sum_{i=1}^{n} \operatorname{sen}\left(2 y_{i}\right), G=\sum_{i=1}^{n} \cos \left(2 w_{i}\right)$ e $H=\sum_{i=1}^{n} \operatorname{sen}\left(2 w_{i}\right)$

$\mathrm{Na}$ Tabela 1.1 encontram-se dados relativos à direção tomada por pombos após 30 e 60 segundos da soltura. O objetivo do experimento era avaliar a possibilidade de existência de alguma estratégia de vôo em animais dessa 
Tabela 1.1: Direção tomada por pombos após 30 e 60 segundos da soltura

\begin{tabular}{|c|c|c|c|c|c|c|c|c|}
\hline \multirow[b]{2}{*}{ Pombo } & \multicolumn{2}{|c|}{$\begin{array}{c}\text { Tempo após } \\
\text { soltura }\end{array}$} & \multirow[b]{2}{*}{ Pombo } & \multicolumn{2}{|c|}{$\begin{array}{c}\text { Tempo após } \\
\text { soltura }\end{array}$} & \multirow[b]{2}{*}{ Pombo } & \multicolumn{2}{|c|}{$\begin{array}{c}\text { Tempo após } \\
\text { soltura }\end{array}$} \\
\hline & 30 & 60 & & 30 & 60 & & 30 & 60 \\
\hline 1 & 2.40 & 250 & 11 & 15 & 10 & 21 & 50 & 50 \\
\hline 2 & 300 & 290 & 12 & 330 & 305 & 22 & 200 & 195 \\
\hline 3 & 225 & 210 & 13 & 100 & 95 & 23 & 330 & 320 \\
\hline 4 & 285 & 325 & 14 & 35 & 65 & 24 & 325 & 315 \\
\hline 5 & 210 & 205 & 15 & 340 & 345 & 25 & 330 & 290 \\
\hline 6 & 265 & 240 & 16 & 320 & 325 & 26 & 280 & 285 \\
\hline 7 & 310 & 330 & 17 & 340 & 335 & 27 & 180 & 155 \\
\hline 8 & 330 & 315 & 18 & 355 & 25 & 28 & 50 & 25 \\
\hline 9 & 325 & 285 & 19 & 40 & 330 & 29 & 20 & 0 \\
\hline 10 & 290 & 335 & 20 & 225 & 220 & & & \\
\hline
\end{tabular}

espécie. Os dados foram coletados em 1982 e correspondem aos pombos soltos no local I, durante o período da manhã e com informações completas. Para a estimativa de $\hat{\rho}_{T}$, tem-se $A=2,659, B=4,733, C=-0,596, D=0,838$, $E=2,139, F=-4,914, G=-3,018$ e $H=-0,756$, resultando em $\hat{\rho}_{T}=0,064$, o que indica uma associação T-toroidal fraca.

Considere $z_{i} \sim \mathcal{N}\left(0, \sigma_{i}^{2}\right), i=1,2$, com Corr $\left(z_{1}, z_{2}\right)=\rho$ e defina $y_{i}=$ $z_{i}(\bmod 2 \pi)$. Então a correlação toroidal entre $y_{1}$ e $y_{2}$ é dada por

$$
\rho_{T}=\frac{\operatorname{senh}\left(2 \rho \sigma_{1} \sigma_{2}\right)}{\left\{\operatorname{senh}\left(2 \sigma_{1}^{2}\right) \operatorname{senh}\left(2 \sigma_{2}^{2}\right)\right\}} .
$$

Outros conceitos de dependência podem ser encontrados em Fisher (1993), Breckling (1989) e Upton e Fingleton (1989).

\subsubsection{Modelos de séries temporais para variáveis cir- culares}

Destacamos nesta seção três modelos de séries temporais desenvolvidos para dados circulares.

Fisher e Lee (1994) desenvolveram modelos de séries temporais circulares criados a partir da transformação das variáveis circulares em lineares. Seja 
$h(\cdot)$ uma função de ligação ímpar em $(-\pi, \pi], \operatorname{com} h(0)=0$. Por definição um processo circular $\left\{y_{i}\right\}$, estacionário, com média circular $\mu$ é denominado processo auto-regressivo de médias móveis ligado de ordem p e q, representado por LARMA(p,q), se $X_{i}=h^{-1}\left(y_{i}-\mu\right)$ for um processo $\operatorname{ARMA}(\mathrm{p}, \mathrm{q})$. Estimado $\mu$ segurido (1.1) e dada a função $h$, a estimação a identificação do modelo podem ser realizadas segundo a teoria usual, levando-se em conta a série $\left\{h^{-1}\left(y_{i}-\mu\right)\right\}$ (ver Brockwell e Davis, 1991, por exemplo).

Outro processo, também discutido em Fisher e Lee (1994), é o circular (pseudo-) auto-regressivo de ordem $p(\mathrm{CAR}(\mathrm{p}))$ que é definido pela série temporal

$$
y_{i} \mid y_{i-1}, y_{i-2}, \ldots \sim \mathcal{M}\left(\mu_{i}, \lambda\right) \quad i=p+1, p+2, \ldots,
$$

onde $\mu_{i}=\alpha+h\left\{\sum_{j=1}^{p} \beta_{j} h^{-1}\left(y_{i-j}-\alpha\right)\right\}$ e $\lambda$ é o parâmetro de concentração. Indica-se o método de máxima verossimilhança para a estimação dos parâmetros. A função de verossimilhança dos dados pode ser facilmente obtida, fixando-se $y_{1}, \ldots, y_{p}$, ou supondo-se $y_{i} \sim \mathcal{M}\left(\mu_{i} ; \lambda\right), i=1,2, \ldots, p$. A princípio, qualquer outra distribuição circular pode ser utilizada para a modelagem da série. Fisher e Lee (1994) discutem a identificação desse tipo de modelo.

Fisher (1993) indica a utilização do modelo LARMA para séries mais concentradas $(\lambda \geq 2)$ e do CAR para séries mais dispersas $(\lambda<2)$.

Breckling (1989) propõe um modelo de série temporal circular, $\left\{y_{i}\right\}$, que seria obtido a partir de um processo auto-regressivo latente estacionário gaussiano $\left\{Z_{i}\right\}$, de tal modo que $y_{i}=Z_{i}(\bmod 2 \pi)$. Nesse caso, toda a modelagem da série seria feita sobre o processo latente $\left\{Z_{i}\right\}$. O modelo foi então denominado de WAR(p), wrapped normal auto-regressive. São propostos métodos de estimação e identificação de modelos.

Accardi et al. (1987) apresentam alguns processos markovianos envolvendo dados circulares.

\subsection{Modelos de dispersão}

Nesta seção apresentamos uma classe ampla de modelos probabilísticos que engloba a distribuição von Mises, dentre outras.

A classe dos modelos de dispersão, Jørgensen (1987), surge naturalmente como uma generalização da distribuição normal (ver Jørgensen, 1997b). 
Considere

$$
f\left(y ; \mu, \sigma^{2}\right)=a\left(\sigma^{2}\right) \exp \left\{-\frac{1}{2 \sigma^{2}} d(y ; \mu)\right\},
$$

onde $a\left(\sigma^{2}\right)=(\sigma \sqrt{2 \pi})^{-1}$ e $d(y ; \mu)=(y-\mu)^{2}$, como sendo a função densidade de probabilidades de uma distribuição normal com média $\mu$ e variância $\sigma^{2}$. Note que o parâmetro $\mu$ aparece apenas no expoente de (1.11), que é negativo, associado a $y$ na forma de uma distância quadrática entre $y$ e $\mu$, ponderada por $\sigma^{2}$. A constante que multiplica a parte exponencial depende apenas de $\sigma^{2}$ e isso faz com que $\mu$ desempenhe o papel de um parâmetro de posição e $\sigma^{2}$ de um parâmetro de escala.

Os modelos de dispersão possuem uma função densidade de probabilidades (ou função de probabilidades no caso discreto) com forma semelhante a (1.11), onde a função $d(\cdot ; \cdot)$ satisfaz certas propriedades, discriminadas na Definição 1.

Definição 1 . Sejam $\Theta \subseteq \mathcal{X} \subseteq \Re$ intervalos com $\Theta$ aberto. Uma função $d: \mathcal{X} \times \Theta \rightarrow \Re$, satisfazendo

$$
d(y ; y)=0, \quad \forall y \in \Theta
$$

$e$

$$
d(y ; \mu)>0, \quad \forall(y, \mu) \in \mathcal{X} \times \Theta, y \neq \mu
$$

é chamada de desvio unitário.

Baseado na definição de desvio unitário, pode-se definir a classe dos modelos de dispersão como a das variáveis aleatórias $y$, cujas funçòes densidade de probabilidade (ou funções de probabilidade, no caso de variáveis discretas) assumem a forma:

$$
p\left(y ; \mu, \sigma^{2}\right)=a\left(y ; \sigma^{2}\right) \exp \left\{-\frac{1}{2 \sigma^{2}} d(y ; \mu)\right\}, y \in \mathcal{X}, \mu \in \Theta \subseteq \mathcal{X}, \sigma^{2}>0
$$

onde $\mathcal{X}$ é um intervalo real, $a(\cdot ; \cdot)$ é uma função, $d(\cdot: \cdot)$ é um desvio unitário e $\mu$ e $\sigma^{2}$ são parâmetros do modelo. Representa-se $y \sim \mathcal{M D}\left(\mu ; \sigma^{2}\right)$. Note que pela forma com que aparecem em (1.12), $\mu$ pode ser interpretado como sendo um parâmetro de posição e $\sigma^{2}$ como um parâmetro de dispersão ou escala. Essa classe de distribuições engloba uma vasta gama de distribuições que vai desde a família exponencial até distribuições de ângulos aleatórios e 
proporções contínuas, dentre outras. Uma sub-família bastante importante é a dos modelos próprios de dispersão, que são aqueles tais que $a\left(y ; \sigma^{2}\right)=$ $c\left(\sigma^{2}\right) b(y)$.

A classe dos modelos de dispersão pode ser generalizada considerando-se variáveis aleatórias com função densidàde de probabilidades (ou função de probabilidades) dada por

$$
p(y ; \mu, \lambda)=c(\lambda ; y) \exp \{\lambda t(y ; \mu)\}, y \in \mathcal{X},
$$

onde $t(\cdot ; \cdot)$ é uma função conveniente, $\mu$ e $\lambda$ são parâmetros do modelo.

\subsubsection{Exemplos de modelos de dispersão}

Nesta seção destacamos alguns exemplos de modelos de dispersão.

\section{Modelos exponenciais de dispersão}

Os modelos exponenciais de dispersão compreendem a família exponencial de distribuições (ver McCullagh e Nelder, 1989, por exemplo). São formados por variáveis aleatórias que possuem função densidade de probabilidades (ou função de probabilidades no caso discreto) dada por

$$
p(y ; \theta, \lambda)=c(y ; \lambda) \exp [\lambda\{\theta y-\kappa(\theta)\}],
$$

onde $E(y)=\mu=\kappa^{\prime}(\theta)=\tau(\theta), \theta=\tau^{-1}(\mu)$ é o parâmetro canônico, $\operatorname{Var}(y)=$ $\lambda^{-1} v(\mu), v(\mu)=\partial \mu / \partial \theta=\kappa^{\prime \prime}(\theta)=\tau^{\prime}(\mu), \lambda^{-1}$ é o parâmet.ro de dispersão $\mathrm{e}$ $c(\cdot, \cdot)$ é uma função conveniente.

Para reescrever (1.14) na forma (1.12), considere $\tilde{\theta}$ como o valor de $\theta$ que maximiza a função de verossimilhança de $\theta$ obtida a partir de (1.14). Esse valor é a solução de

$$
y-\tau(\tilde{\theta})=0 \Rightarrow \tilde{\theta}=\tau^{-1}(y)
$$

Defina

$$
\begin{aligned}
d(y ; \mu) & =2\left[y \tilde{\theta}-\kappa(\tilde{\theta})-y \tau^{-1}(\mu)+\kappa\left\{\tau^{-1}(\mu)\right\}\right] \\
& =2\left[y \tau^{-1}(y)-\kappa\left\{\tau^{-1}(y)\right\}-y \tau^{-1}(\mu)+\kappa\left\{\tau^{-1}(\mu)\right\}\right] .
\end{aligned}
$$

É fácil ver que $d$ é um desvio unitário. Assim, definindo $\sigma^{2}=\lambda^{-1}$, pode-se reescrever (1.14) como

$$
p\left(y ; \mu, \sigma^{2}\right)=a\left(y ; \sigma^{2}\right) \exp \left\{-\frac{1}{2 \sigma^{2}} d(y: \mu)\right\} .
$$


onde

$$
a\left(y ; \sigma^{2}\right)=c\left(y ; \sigma^{-2}\right) \exp \left[\sigma^{-2}\{y \tilde{\theta}-\kappa(\tilde{\theta})\}\right] .
$$

Isso demonstra que a família exponencial faz parte da classe dos modelos de dispersão. Maiores detalhes podem ser encontrados em Jørgensen (1987 e i99?b).

\section{Distribuição von Mises}

Outro exemplo de modelo de dispersão é a distribuição von Mises. Definindo-se $\sigma^{2}=\lambda^{-1}$, a densidade (1.3) pode ser reescrita na forma (1.12), como

$$
p\left(y ; \mu, \sigma^{2}\right)=\frac{e^{\sigma^{-2}}}{2 \pi I_{0}\left(\sigma^{-2}\right)} \exp \left[-\frac{1}{2 \sigma^{2}} 2\{1-\cos (y-\mu)\}\right] .
$$

\section{Distribuições Simplex}

As distribuições simplex (Barndorff-Nielsen e Jørgensen, 1991) formam uma classe de modelos que assumem valores no intervalo unitário e que possuem função densidade de probabilidades dada por

$$
p\left(y ; \mu, \sigma^{2}, \alpha\right)=a_{\alpha}\left(\mu ; \sigma^{2}\right) y^{\alpha_{1}-1}(1-y)^{\alpha_{2}-1} \exp \left\{-\frac{d_{\alpha}(y ; \mu)}{2 \sigma^{2}}\right\}
$$

onde $\mu$ é um parâmetro de posição, $\sigma^{2}$ de dispersão, $\alpha=\left(\alpha_{1}, \alpha_{2}\right)^{\top}$ são parâmetros de forma, $a_{\alpha}(\cdot ; \cdot)$ é uma função conveniente e

$$
d_{\alpha}(y ; \mu)=\mu^{2 \alpha_{1}-1}(1-\mu)^{2 \alpha_{2}-1} \frac{(y-\mu)^{2}}{y(1-y)} .
$$

No caso em que $\alpha$ é conhecido e $a_{\alpha}\left(\mu ; \sigma^{2}\right)$ não depende de $\mu$ a distribuição (1.17) pertence à família dos modelos de dispersão. Dentre as distribuições simplex, destaca-se a simplex padrão, que é obtida quando $\alpha=(-0,5 ;-0,5)$. Nesse caso, sua função densidade de probabilidades assume a forma

$$
p\left(y ; \mu, \sigma^{2}\right)=\left[2 \pi \sigma^{2}\{y(1-y)\}^{3}\right]^{-1 / 2} \exp \left\{-\frac{1}{2 \sigma^{2}} d(y: \mu)\right\},
$$

com

$$
d(y ; \mu)=\frac{(y-\mu)^{2}}{y(1-y) \mu^{2}(1-\mu)^{2}} .
$$




\subsubsection{Inferências para um modelo de dispersão}

A fim de facilitar a notação de resultados futuros, é conveniente definir dois operadores matriciais. Considere o vetor real $\mathrm{s}=\left(s_{1}, s_{2}, \ldots, s_{c}\right)^{\top}$ e a matriz real $\mathbf{S}$ com componentes $s_{i j}, i, j=1,2, \ldots, c$. Defina os seguintes operadores:

a. $\Delta(\cdot)$, que aplicado a um vetor retorna uma matriz diagonal $c$-dimensional, tal que $\Delta(\mathrm{s})=\operatorname{diag}\left\{s_{1}, s_{2}, \ldots, s_{c}\right\} \mathrm{e}$

b. $\delta(\cdot)$, aplicado a uma matriz quadrada, onde $\delta(\mathbf{S})=\left(s_{11}, s_{22}, \ldots, s_{c c}\right)^{\top}$.

Considere $\left(y_{i}, \mathbf{x}_{i}\right), y_{i} \sim \mathcal{M D}\left(\mu_{i} ; \sigma^{2}\right), i=1,2, \ldots, n$, uma amostra aleatória, onde $\mathbf{x}_{i}$ é um vetor $p$-dimensional de covariáveis fixas. Seja $g$ uma função de ligação, duplamente diferenciável, inversível e tal que $g\left(\mu_{i}\right)=\mathbf{x}_{i}^{\top} \boldsymbol{\beta}=\eta_{i}$, onde $\boldsymbol{\beta}$ é um vetor de parâmetros.

Para modelos que satisfazem às condições usuais de regularidade, a função escore e a matriz de informação de Fisher associadas à $\boldsymbol{\beta}$ são dadas, respectivamente, por

$$
\begin{aligned}
\mathbf{U}(\boldsymbol{\beta}) & =-\frac{1}{2 \sigma^{2}} \mathbf{X}^{\top} \mathbf{H u} \text { e } \\
\mathbf{I}_{F}(\boldsymbol{\beta}) & =-\frac{1}{2 \sigma^{2}} \mathbf{X}^{\top} \mathbf{H} E\{\Delta(\dot{\mathbf{u}})\} \mathbf{H X}=\frac{1}{4 \sigma^{4}} \mathbf{X}^{\top} \mathbf{H} E\left(\mathbf{u u}^{\top}\right) \mathbf{H X},
\end{aligned}
$$

onde $\mathbf{u}=\left(u_{i}, \ldots, u_{n}\right)^{\top}, u_{i}=\frac{\partial d}{\partial \mu_{i}}, \dot{\mathbf{u}}=\left(\frac{\partial u}{\partial \mu_{1}}\left(\mathbf{y}_{1}, \mu_{1}\right), \ldots, \frac{\partial u}{\partial \mu_{n}}\left(\mathbf{y}_{n}, \mu_{n}\right)\right)^{\top}$, $\mathbf{H}=\operatorname{diag}\left\{\frac{\partial g^{-1}}{\partial \eta_{1}}\left(\eta_{1}\right), \ldots, \frac{\partial g^{-1}}{\partial \eta_{n}}\left(\eta_{n}\right)\right\}$ e $\mathbf{X}=\left(\mathbf{x}_{1}, \mathbf{x}_{2}, \ldots, \mathbf{x}_{n}\right)^{\top}$.

Sob condições gerais de regularidade, as raízes da funçào escore são estimadores consistentes, assintoticamente normais, com matriz de covariância amostral dada pelo inverso da matriz de informação de Fishẹ.

Pode-se construir testes de hipóteses sobre $\boldsymbol{\beta}$ baseados na distribuição assintótica do estimador (por exemplo: teste de Wald). Outra abordagem, desenvolvida para hipóteses encaixadas, semelhante a utilizada na teoria de modelos lineares generalizados (ver McCullagh e Nelder. 1989), pode ser empregada para modelos de dispersão em situações especiais. Defina a função desvio, associada à amostra, para $\mu$ como $D=\sum_{i=1}^{n} d\left(y_{i}: \mu_{i}\right)$ e considere as hipóteses $H_{i}: \boldsymbol{\mu} \in \Omega_{i}, i=1,2$, com $\Omega_{1} \subset \Omega_{2}$. O teste de $H_{1}$ contra $H_{2}$ pode ser feito através da estatística

$$
F=\frac{\left(D_{1}-D_{2}\right) /\left(f_{1}-f_{2}\right)}{D_{2} / f_{2}}
$$


onde $D_{i}$ é o desvio total calculado sob a hipótese $H_{i}$, isto é, aquele obtido ao se maximizar a verossimilhança sob $H_{i}$ e $f_{i}, i=1,2$, corresponde ao número de graus de liberdade associado à $H_{i}$. Para $\sigma^{2}$ próximo de zero ou para $n$ suficientemente grande, a distribuição de $F$ aproxima-se de uma $F\left(f_{1}-f_{2}, f_{2}\right)$ (ver Jørgensen, 1997b, por exemplo).

Uma propriedade importante dos modelos de dispersão é a ortogonalidade entre os parâmetros de posição e dispersão. Em termos práticos essa relação traz simplificações ao processo de estimação desses parâmetros (ver Jørgensen, 1997b, por exemplo).

\section{Estimador de máxima verossimilhança para $\sigma^{2}$}

O estimador de máxima verossimilhança para $\sigma^{2}$ pode ser facilmente obtido a partir da função log-verossimilhança, sendo dado pela raiz de

$$
\sum_{i=1}^{n} \frac{\dot{a}_{i}}{a_{i}}+\frac{1}{2 \sigma^{4}} \sum_{i=1}^{n} d_{i}
$$

onde $a_{i}=a\left(y_{i} ; \sigma^{2}\right), \dot{a}_{i}=\frac{\partial a_{i}}{\partial \sigma^{2}}$, avaliada em $\sigma^{2}$ e $d_{i}=d\left(y_{i} ; \mu_{i}\right)$.

Sob condições de regularidade, prova-se a consistência e a normalidade assintótica desse estimador.

\section{Exemplos}

Para modelos exponenciais de dispersão, tem-se que o estimador de máxima verossimilhança de $\sigma^{2}$ é a raiz de

$$
\sum_{i=1}^{n} \frac{\dot{c}_{i}}{c_{i}}-\frac{1}{2 \sigma^{4}} \sum_{i=1}^{n}\left\{\theta_{i} y_{i}-\kappa\left(\theta_{i}\right)\right\}
$$

onde $c_{i}=c\left(y_{i} ; \sigma^{2}\right)$, definido em (1.14) e $\dot{c}_{i}=\frac{\partial c_{i}}{\partial \sigma^{2}}$, avaliada em $\sigma^{2}$.

Esse estimador terá uma forma bastante simplificada no caso da distribuição von Mises. Considere a função densidade definida em (1.3). O estimador de máxima verossimilhança para $\lambda$ é dado pela soluçào de

$$
\mathcal{A}_{1}(\hat{\lambda})=\frac{\sum_{i=1}^{n} \cos \left(y_{i}-\hat{\mu}_{i}\right)}{n} .
$$

Para calcular o valor da função inversa de $\mathcal{A}_{1}$, basta proceder como em (A.2). 


\section{Estimador de Pearson para $\sigma^{2}$}

Jørgensen (1997b) generaliza a função de variância, anteriormente definida para modelos esponenciais de dispersão, para toda a classe dos modelos de dispersão como

$$
v\left(\mu_{i} ; \sigma^{2}\right)=2 / E\left(\dot{u}_{i}\right),
$$

com $\dot{u}_{i}=\partial u_{i} / \partial \mu_{i}$. Baseado nessa função, define-se a estatística generalizada de Pearson por

$$
X^{2}\left(\boldsymbol{\mu}, \sigma^{2}\right)=\sum_{i=1}^{n} v\left(\mu_{i} ; \sigma^{2}\right) \tilde{u}^{2}\left(y_{i} ; \mu_{i}\right)
$$

onde $\tilde{u}\left(y_{i} ; \mu_{i}\right)=u_{i} / 2$. Sob a suposição de regularidade do modelo de dispersão, demonstra-se que

e portanto

$$
\operatorname{Var}\left(u_{i}\right)=E\left(u_{i}^{2}\right)=\frac{\sigma^{2}}{v\left(\mu_{i} ; \sigma^{2}\right)},
$$

$$
E\left\{X^{2}\left(\boldsymbol{\mu}, \sigma^{2}\right)\right\}=n \sigma^{2} .
$$

$\mathrm{O}$ estimador de Pearson para $\sigma^{2}$ é definido por

$$
\hat{\sigma}^{2}=\frac{1}{n-p} X^{2}\left(\hat{\boldsymbol{\mu}}, \sigma^{2}\right)
$$

Demonstra-se que esse estimador é assintoticamente não-viciado e consistente.

\section{Exemplos}

Em um modelo exponencial de dispersão tem-se

$$
u_{i}=-2 v^{-1}\left(\mu_{i}\right)\left(y_{i}-\mu_{i}\right)
$$

e $v\left(\mu_{i} ; \sigma^{2}\right)=v\left(\mu_{i}\right)$, conforme definido na Seção 1.2.1. Desse modo o estimador de Pearson para $\sigma^{2}$ é dado por

$$
\bar{\sigma}^{2}=\sum_{i=1}^{n} \frac{\left(y_{i}-\hat{\mu}_{i}\right)^{2}}{v\left(\mu_{i}\right)} .
$$

Já para a distribuição von Mises, tem-se $u_{i}=-2 \operatorname{sen}\left(y_{\imath}-\mu_{i}\right)$ e. consequentemente, $v\left(\mu_{i} ; \sigma^{2}\right)=1 / \mathcal{A}_{1}\left(\sigma^{-2}\right)$. Assim, o estimador de Pearson para $\sigma^{2}$ é a solução de

$$
\sigma^{2}=\sum_{i=1}^{n} \frac{\operatorname{sen}^{2}\left(y_{i}-\mu_{i}\right)}{\mathcal{A}_{1}\left(\sigma^{-2}\right)} .
$$




\subsection{Apresentação dos capítulos}

O Capítulo 2 trata da teoria das funções de estimação, onde são apresentados algurs resultados assintóticos originais para estimadores obtidos a partir delas. No Capítulo 3, desenvolvemos várias funções de estimação para a análise de modelos de dispersão longitudinais. Particularmente damos especial atenção às desenvolvidas para a análise de dados circulares. No Capítulo 4 , sugerimos a utilização de uma distribuição circular multivariada (normal arqueada multivariada) para a modelagem de dados circulares longitudinais e apresentamos várias simulações envolvendo aspectos ligados à teoria desenvolvida. No Capítulo 5 aplicamos parte da metodologia desenvolvida na tese a um conjunto de dados reais. Por fim, no Capítulo 6 apresentamos nossas conclusões e sugestões para futuros estudos. 


\section{Capítulo 2}

\section{Funções de estimação}

Neste capítulo introduzimos a definição de função de estimação e apresentamos uma série de resultados relacionados a propriedades assintóticas de estimadores obtidos através de equações de estimação.

Uma função de estimação é, de um modo simplificado, uma função dos dados e dos parâmetros de interesse. Em termos práticos, elas são construídas de modo a que suas raízes, quando existem, sejam estimativas dos parâmetros envolvidos. Um ponto importante no estudo dessas funções é o estabelecimento de condições que garantam que os estimadores obtidos possuam boas propriedades. Em geral, deseja-se a construção de estimadores consistentes e com distribuição assintótica conhecida.

Seja $(\mathcal{X}, \mathcal{A})$ um espaço mensurável, sobre o qual define-se um modelo estatístico $(\mathcal{X}, \mathcal{A}, \wp), \operatorname{com} \mathcal{X} \in \mathcal{R}$ e onde $\wp=\left\{P_{\theta}: \theta \in \Theta \subseteq \mathcal{R}^{p}\right\}$ e seja $\nu$ uma medida $\sigma$-finita sobre $(\mathcal{X}, \mathcal{A})$. Por definição, uma funçào $\psi: \mathcal{X} \times \Theta \rightarrow$ $\mathcal{R}^{p}$, é uma função de estimação se para cada $\theta \in \Theta, \psi(. . \theta)$ é uma funçào mensurável.

Assumindo a existência de uma amostra de $n$ vetores aleatórios independentes: $\mathbf{y}_{i}=\left(y_{i 1}, y_{i 2}, \ldots, y_{i t}\right)^{\top}, i=1,2, \ldots, n$, onde a cada unidade amostral $i$ associa-se uma função de estimação $\psi_{i}$, estende-se o conceito de funçào de estimação para a amostra através de $\boldsymbol{\Psi}_{n}(\mathrm{y} ; \boldsymbol{\theta})=\sum_{i=1}^{n} \psi_{i}\left(\mathrm{y}_{i}: \theta\right)$.

Considere entào uma amostra aleatória $y_{i}, i=1.2 \ldots \ldots$. onde $p\left(y_{i} ; \theta\right)$ é a funçâo densidade de probabilidade regular associada a $y_{i}$; nessas condições, a função escore

$$
\boldsymbol{\Psi}_{n}(\mathrm{y} ; \boldsymbol{\theta})=\sum_{i=1}^{n} \frac{\partial}{\partial \boldsymbol{\theta}} \log p\left(y_{i} ; \boldsymbol{\theta}\right),
$$


onde $\mathbf{y}=\left(y_{1}, y_{2}, \ldots, y_{n}\right)^{\top}$, é uma função de estimação. Outra função de estimação bastante utilizada é obtida supondo-se $E\left(y_{i}\right)=\mu_{i}(\theta)$ e tomando-se

$$
\boldsymbol{\Psi}_{n}(\mathbf{y} ; \boldsymbol{\theta})=\sum_{i=1}^{n} \frac{\partial \mu_{i}}{\partial \boldsymbol{\theta}}(\boldsymbol{\theta})\left\{y_{i}-\mu_{i}(\boldsymbol{\theta})\right\}
$$

As raízes dessa função são os estimadores de mínimos quadrados de $\boldsymbol{\theta}$.

Como foi salientado, é necessário o estabelecimento de condições sob as quais as raízes de uma função de estimação possuam boas propriedades assintóticas. Nessa direção, surgem os conceitos de função de estimação regular e de informação de Godambe, que serão explicitados a seguir. Por facilidade de notação, para $n$ igual a $u m$, denotaremos a função de estimação por $\boldsymbol{\Psi}(y ; \boldsymbol{\theta})$ sempre que isso não prejudicar a clareza do texto.

Definição 2 . Uma funçâo $\boldsymbol{\Psi}(y ; \boldsymbol{\theta})=\left(\Psi_{1}(y ; \boldsymbol{\theta}), \Psi_{2}(y ; \boldsymbol{\theta}), \ldots, \Psi_{p}(y ; \boldsymbol{\theta})\right)^{\top}$, $\Psi: \mathcal{X} \times \Theta \rightarrow \mathcal{R}^{p}$ é dita regular se para todo $\theta=\left(\theta_{1}, \ldots, \theta_{p}\right)^{\top} \in \Theta$,

i. a função é não viciada, $E_{\boldsymbol{\theta}}\{\boldsymbol{\Psi}(y ; \boldsymbol{\theta})\}=0$;

ii. a derivada parcial $\partial \Psi(y ; \theta) / \partial \theta_{i}$ existe quase certamente para $y \in \mathcal{X}$;

iii. é possível permutar o sinal de integração e diferenciação da seguinte forma:

$$
\frac{\partial}{\partial \theta_{i}} \int_{\mathcal{X}} \boldsymbol{\Psi}(y ; \boldsymbol{\theta}) p(y ; \boldsymbol{\theta}) d \nu(y)=\int_{\mathcal{X}} \frac{\partial}{\partial \theta_{i}}\{\boldsymbol{\Psi}(y ; \boldsymbol{\theta}) p(y ; \boldsymbol{\theta})\} d \nu(y)
$$

iv. $E_{\boldsymbol{\theta}}\left\{\Psi_{i}(y ; \boldsymbol{\theta}) \Psi_{j}(y ; \boldsymbol{\theta})\right\} \in \mathcal{R}$, para $i, j=1, \ldots, p \in$

$$
\mathrm{V}_{\boldsymbol{\Psi}}(\boldsymbol{\theta})=E_{\boldsymbol{\theta}}\left\{\boldsymbol{\Psi}(y ; \boldsymbol{\theta}) \bar{\Psi}^{\top}(y ; \boldsymbol{\theta})\right\}
$$

é positiva definida e

v. $E_{\boldsymbol{\theta}}\left\{\frac{\partial \psi_{i}}{\partial \theta_{r}}(y ; \boldsymbol{\theta}) \frac{\partial \psi_{j}}{\partial \theta_{s}}(y ; \boldsymbol{\theta})\right\} \in \mathcal{R}$ e $\mathbf{S}_{\boldsymbol{\Psi}}(\boldsymbol{\theta})=E_{\boldsymbol{\theta}}\left\{\nabla_{\boldsymbol{\theta}} \boldsymbol{\Psi}(y ; \boldsymbol{\theta})\right\}$ é nâo singular, onde $\nabla_{\theta}$ representa o operador gradiente $\mathrm{em}$ rtação a $\theta$.

Definição 3 . Seja $\boldsymbol{\Psi}(\mathbf{y} ; \boldsymbol{\theta})$ uma função de estimaçào reguiar. Define-se a matriz de informação de Godambe de $\theta$ associada a $\Psi$ por

$$
\mathrm{J}_{\Psi}(\theta)=\mathrm{S}_{\Psi}(\theta) \mathrm{V}_{\Psi}^{-1}(\theta) \mathrm{S}_{\Psi}(\theta) .
$$


A informação de Godambe (ver Jørgensen e Laboriau, 1994, por exemplo) desempenha o papel da informação de Fisher para as funções de estimação regulares.

A componente $\mathbf{S}_{\Psi}(\boldsymbol{\theta})$ da matriz de informação de Godambe está relacionada à derivada parcial da função de estimação em relaçâo aos parâmetros. É desejável que uma pequena variação no vetor paramétrico acarrete uma grande variação no valor da função de estimação, pois quanto maior essa variação, maior eficiência a função terá na estimação do parâmetro. No caso unidimensional espera-se que $S(\theta)$ assuma um valor alto. $\mathbf{S}(\theta)$ é denominada de sensitividade da função de estimação.

A função escore, obtida a partir de uma função densidade de probabilidade (ou função de probabilidade, no caso discreto) regular, satisfaz as propriedades da Definição 2 e, além disso, $S_{\Psi}(\theta)=-V_{\Psi}(\theta)$, o que faz com que sua matriz de informação de Godambe coincida com a matriz de informação de Fisher.

O próximo teorema estabelece condições para a normalidade assintótica de estimadores obtidos a partir de funções de estimação regulares.

Teorema 1. (Jørgensen e Laboriau, 1994). Seja $\theta$ um parâmetro unidimensional $e$ considere a amostra aleatória $y_{i}, i=1,2, \ldots, n$, com $\mathbf{y}=$ $\left(y_{1}, y_{2}, \ldots, y_{n}\right)^{\top}$. Uma sequência de raízes $\left\{\hat{\theta}_{n}\right\}_{n=1}^{\infty}$ associada a uma função de estimação regular $\Psi_{n}(\mathbf{y} ; \theta)=\sum_{i=1}^{n} \psi_{i}\left(y_{i} ; \theta\right)$ e tal que

$$
\hat{\theta}_{n} \stackrel{\mathcal{P}}{\rightarrow} \theta
$$

é assintoticamente normal

$$
\sqrt{n}\left(\hat{\theta}_{n}-\theta\right) \stackrel{\mathcal{D}}{\rightarrow} \mathcal{N}\left(0, \bar{J}_{\Psi}^{-1}(\theta)\right),
$$

onde

$$
\bar{J}_{\Psi}=S^{2}(\theta) / V(\theta)
$$

e

$$
\begin{aligned}
& S(\theta)=\lim _{n \rightarrow \infty} \sum_{i=1}^{n} \frac{S_{\psi_{i}}}{n}(\theta) ; \quad S_{\psi_{i}}(\theta)=E_{\theta}\left\{\nabla_{\theta \psi_{i}}\left(y_{i}: \theta\right)\right\}: \\
& V(\theta)=\lim _{n \rightarrow \infty} \sum_{i=1}^{n} \frac{V_{\psi_{i}}}{n}(\theta) \text { e } V_{\psi_{\mathrm{i}}}(\theta)=E_{\theta}\left\{\zeta_{\mathrm{i}}^{2}\left(y_{\mathrm{i}}: \theta\right)\right\} .
\end{aligned}
$$


Examinando-se o Teorema 1, nota-se que, no caso do parâmetro ser unidimensional, dada uma função de estimação regular, basta provar a consistência de suas raízes para que sua distribuição assintótica seja conhecida. Há na literatura estatística uma grande variedade de resultados nessa direção. $\mathrm{Na}$ Seção 2.2, apresentamos resultados por nós desenvolvidos para. o caso de parâmetro multidimensional.

No caso de $\theta$ ser multidimensional, sob condiçôes de regularidade, a matriz

$$
\overline{\mathbf{J}}_{\Psi}=\mathbf{S}(\theta) \mathbf{V}^{-1}(\theta) \mathbf{S}^{\top}(\theta),
$$

onde

$$
\begin{gathered}
\mathbf{S}(\boldsymbol{\theta})=\lim _{n \rightarrow \infty} \sum_{i=1}^{n} \frac{\mathbf{S}_{\boldsymbol{\psi}_{i}}(\boldsymbol{\theta})}{n} ; \quad \mathbf{S}_{\boldsymbol{\psi}_{i}}(\boldsymbol{\theta})=E_{\boldsymbol{\theta}}\left\{\nabla_{\boldsymbol{\theta}} \boldsymbol{\psi}_{i}\left(y_{i} ; \boldsymbol{\theta}\right)\right\} \\
\mathbf{V}(\boldsymbol{\theta})=\lim _{n \rightarrow \infty} \sum_{i=1}^{n} \frac{\mathbf{V}_{\boldsymbol{\psi}_{i}}(\boldsymbol{\theta})}{n} \mathrm{e} \mathbf{V}_{\boldsymbol{\psi}_{i}}(\boldsymbol{\theta})=E_{\boldsymbol{\theta}}\left\{\boldsymbol{\psi}_{i}\left(y_{i} ; \boldsymbol{\theta}\right) \boldsymbol{\psi}_{i}^{\top}\left(y_{i} ; \boldsymbol{\theta}\right)\right\}
\end{gathered}
$$

desempenha $\circlearrowleft$ papel de uma matriz de informação de Godambe assintótica associada a $\boldsymbol{\Psi}_{n}$.

Como textos introdutórios ao assunto, recomenda-se Godambe (1960), Godambe e Kale (1991), Godambe (1991), McLeish e Small (1988). Recomenda-se também a leitura de Liang e Zeger (1995), por se tratar de um artigo mais recente.

Para simplificar a notação, a partir de agora, os argumentos y e $\mathrm{y}_{i}$ das funções de estimação serão omitidos.

\subsection{Função de estimação ótima}

Um conceito importante, desenvolvido por Godambe (1960). é o de otimalidade de uma função de estimação regular. No caso $\theta$ unidimensional, pode-se definir uma função de estimação ótima como aquela cujas raizes possuem variância assintótica mínima. Esse conceito pode ser estendido para o caso multidimensional através da introdução de alguma ordenação das matrizes de covariância assintóticas.

Definição 4. Seja $\mathbf{J}_{\Psi}^{-1}$ a matriz de covariância assintótica de um estimador $\hat{\boldsymbol{\theta}}$ obtido a partir de uma função de estimaçâo $\boldsymbol{\Psi}$. Se existir uma funçâo de estimaçấo $\Psi^{*}$ que gera um estimador de $\theta$ com matriz de covariância assintótica $\mathbf{J}_{\Psi^{*}}^{-1}$, tal que $\mathbf{J}_{\Psi}^{-1}-\mathbf{J}_{\Psi^{*}}^{-1}$ seja não-negativa definida para qualquer $\boldsymbol{\Psi}$, então $\Psi^{*}$ é dita ser uma funçâo de estimaçào ótima para $\theta$. 
Chandrasekar and Kale (1984) provam que, sob condições gerais, as funções de estimação ótimas obtidas pelos critérios $\operatorname{tr}\left(\mathbf{J}_{\Psi}^{-1}\right) \geq \operatorname{tr}\left(\mathbf{J}_{\Psi^{*}}^{-1}\right)$ e $\left|\mathbf{J}_{\Psi}^{-1}\right| \geq$ $\left|\mathbf{J}_{\Psi^{*}}^{-1}\right|{ }^{1}$ coincidem com a da Definição 4, desde que exista uma ót,ima segundo esses critérios. Pode-se demonstrar também que, no caso regular, a função escore sempre será ótima. Isso faz com que a matriz de informøção de Fisher, quando existir, cumpra o papel de limite superior para as matrizes de informação de Godambe.

É importante ressaltar que se $\Psi_{n}^{*}$ é uma função de estimação ótima, então $\boldsymbol{\Psi}_{n}=\mathbf{M}(\boldsymbol{\theta}) \boldsymbol{\Psi}_{n}^{*}$, onde $\mathbf{M}(\boldsymbol{\theta})$ é uma matriz não-singular, também o será.

Crowder(1987) estuda uma classe particular de funçôes de estimação definidas por $\sum_{i=1}^{n} \mathbf{Q}_{i}(\theta) \mathbf{u}_{i}\left(\mathbf{y}_{i}, \boldsymbol{\theta}\right)$, onde $\mathbf{Q}_{i}$ é uma matriz não estocástica de pesos que, eventualmente, pode ser função de $\boldsymbol{\theta}$, enquanto $\mathbf{u}_{i}, i=1,2, \ldots, n$, são vetores com média zero, mutuamente independentes. Por simplicidade, chame essa classe de $\mathcal{L}$. $\mathrm{O}$ autor mostra que a função de estimação ótima dentre as da classe $\mathcal{L}$ é obtida quando a matriz de pesos é dada por

$$
\mathrm{Q}_{i}^{*}(\boldsymbol{\theta})=E_{\boldsymbol{\theta}}\left(\frac{\partial \mathbf{u}_{i}}{\partial \boldsymbol{\theta}}\right)^{\top} \operatorname{Cov}_{\boldsymbol{\theta}}^{-1}\left(\mathbf{u}_{i}\right)
$$

Denomine $\mathbf{u}_{i}, i=1,2, \ldots, n$, como funções de estimação geradoras da classe $\mathcal{L}$.

Para exemplificar uma aplicação desse resultado, considere uma amostra de variáveis aleatórias independentes, $y_{i}, i=1,2, \ldots, n$, de tal modo que $E\left(y_{i}\right)=\mu_{i}=h_{i}\left(\mathrm{x}_{i}^{\top} \boldsymbol{\beta}\right)=h_{i}\left(\eta_{i}\right)$ e $\operatorname{Var}\left(y_{i}\right)=\sigma^{2} c_{i}\left(\mu_{i}\right)$, onde $\mathrm{x}_{i}$ é um vetor de covariáveis associado a $y_{i} ; \boldsymbol{\beta}$ é um vetor $p$-dimensional de parâmetros desconhecidos; $h_{i}(\cdot)$ é uma função duplamente diferenciável e inversível e $c_{i}(\cdot)$ é uma função positiva, $i=1,2, \ldots, n$. Considere também a fannília de funçôes de estimação dada por $\boldsymbol{\Psi}_{n}(\boldsymbol{\beta})=\mathrm{Q}(\boldsymbol{\beta}) \mathbf{u}(\mathbf{y}, \boldsymbol{\beta})$, onde $\mathbf{Q}(\boldsymbol{\beta})$ é umá funçào de $\boldsymbol{\beta}$ e $\mathbf{u}(\mathbf{y}, \boldsymbol{\beta})=\mathbf{y}-\boldsymbol{\mu}$, com $\mathbf{y}=\left(y_{1}, y_{2}, \ldots, y_{n}\right)$ e $\boldsymbol{\mu}=\left(\mu_{1}, \mu_{2}, \ldots, \mu_{n}\right)$. Obviamente tais funções pertencem à classe $\mathcal{L}$ e assim. utilizando (2.1), vem que a função de estimação ótima nesta classe é

$$
\Psi_{n}^{*}(\boldsymbol{\beta})=-\sigma^{2} \mathbf{X}^{\top} \mathbf{H C}^{-1}(\mathrm{y}-\boldsymbol{\mu})
$$

onde $\mathbf{X}=\left(\mathbf{x}_{1}, \mathbf{x}_{2}, \ldots, \mathbf{x}_{n}\right)^{\top}, \mathbf{H}=\operatorname{diag}\left\{\frac{\partial \mu_{1}}{\partial \eta_{1}}, \ldots, \frac{\partial \mu_{n}}{\partial \eta_{n}}\right\}$ e. por fim, $\mathbf{C}=$ $\operatorname{diag}\left\{c_{1}\left(\mu_{1}\right), c_{2}\left(\mu_{2}\right), \ldots, c_{n}\left(\mu_{n}\right)\right\}$. Note que $(2.2)$ equivale às equações de estimação sugeridas na teoria da quase-verossimilhança. desenvolvida por Wedderburn (1974).

\footnotetext{
${ }^{1}$ onde $|\cdot|$ indica o determinante de uma matriz quadrada
} 
Nas próximas seções os índices das matrizes $\mathrm{S}_{\boldsymbol{\Psi}}$ e $\mathrm{V}_{\boldsymbol{\Psi}}$ serào omitidos, sempre que a omissão não prejudicar a clareza do texto.

\subsection{Alguns resultados assintóticos}

Nesta seção apresentamos resultados assintóticos por nós desenvolvidos, que estabelecem condições sob as quais os estimadores obtidos a partir de uma função de estimação regular são consistentes e assintoticamente normais. $\mathrm{Na}$ Seção 2.2.3 são apresentadas as demonstrações destes teoremas.

Considere que o espaço paramétrico, $\Theta$, é denso, o espaço amostral é euclidiano e que a norma utilizada seja a euclidiana ou a do máximo do módulo das componentes da matriz.

Teorema 2 . Considerando-se que

a. $\mathbf{y}_{i}=\left(y_{i 1}, \ldots, y_{i t}\right)^{\top}, i=1,2, \ldots, n$, säo vetores $t$-dimensionais, independentes e identicamente distribuídos;

b. existe uma função mensurável $f: \mathcal{X} \times \Theta \rightarrow \Theta$, tal que $\psi_{i}(\theta)=f\left(\mathrm{y}_{i}, \theta\right)$, $i=1,2, \ldots, n$;

c. $\boldsymbol{\psi}_{i}(\boldsymbol{\theta}), i=1,2, \ldots, n$, é uma função de estimação regular;

d. $\Psi_{n}(\theta)=\sum_{i=1}^{n} \psi_{i}(\theta)$

e. $\operatorname{para} \delta>0, E_{\boldsymbol{\theta}}\left\{\sup _{\mathbf{h}:\|\mathbf{h}\| \leq \delta}\left\|\frac{\partial \boldsymbol{\psi}_{i}}{\partial \boldsymbol{\theta}}(\boldsymbol{\theta}+\mathbf{h})-\frac{\partial \boldsymbol{\psi}_{i}}{\partial \boldsymbol{\theta}}(\boldsymbol{\theta})\right\|\right\}=\phi_{s}$ c Os $\rightarrow 0$ quando $\delta \rightarrow 0, i=1,2, \ldots, n$;

f. $\left\{\hat{\boldsymbol{\theta}}_{n}\right\}_{n=1}^{\infty}$ é uma sequência de raízes de $\boldsymbol{\Psi}_{n}(\mathrm{w}), \mathrm{w} \in \Theta$

e sob condições que garantam a existência de uma sequência de raízes de $\Psi_{n}(\mathbf{w})$ que seja limitada em probabilidade, ou restrita a um conjunto compacto q.c. quando $n$ tende a infinito ${ }^{2}$, vem que $\hat{\theta}_{n} \stackrel{P}{\rightarrow} \theta$, quando $n \rightarrow \infty$.

\footnotetext{
${ }^{2}$ na Seção 2.2.1, são explicitadas algumas condições que garantem esses resultados
} 
Corolário 1 . Sob as condiçôes do Teorema 2

$$
\sqrt{n}\left(\hat{\theta}_{n}-\theta\right) \stackrel{\mathcal{D}}{\rightarrow} \mathcal{N}_{p}\left(0 ; \mathbf{J}_{\psi}^{-1}\right)
$$

onde $\mathbf{J}_{\psi}$ é a matriz de informação de Godambe de $\psi_{1}$.

A fim de generalizar os resultados anteriores para o caso de vetores resposta mutuamente independentes, mas não identicamente distribuídos, é necessária a adição de novas hipóteses. Isso é feito nos teoremas seguintes.

Teorema 3 . Considerando-se

a. $\mathbf{y}_{i}, i=1,2, \ldots, n$, vetores aleatórios $t$-dimensionais independentes;

b. $\boldsymbol{\psi}_{i}(\boldsymbol{\theta})=\left(\boldsymbol{\psi}_{i 1}, \boldsymbol{\psi}_{i 2}, \ldots, \boldsymbol{\psi}_{i p}\right)^{\top}, i=1,2, \ldots, n$, funções de estimação regulares;

c. $\Psi_{n}(\theta)=\sum_{i=1}^{n} \psi_{i}(\theta)$;

d. ara $\delta>0$,

$$
\begin{aligned}
& E_{\boldsymbol{\theta}}\left\{\sup _{\mathbf{h}:\|\mathbf{h}\| \leq \delta}\left\|\frac{\partial \psi_{i}}{\partial \boldsymbol{\theta}}(\boldsymbol{\theta}+\mathbf{h})-\frac{\partial \boldsymbol{\psi}_{i}}{\partial \boldsymbol{\theta}}(\boldsymbol{\theta})\right\|\right\}=\phi_{\delta i}, \\
& \frac{1}{n} \sum_{i=1}^{n} \phi_{\delta i} \rightarrow \phi_{\delta} \in \phi_{\delta} \rightarrow 0 \text { quando } \delta \rightarrow 0
\end{aligned}
$$

e. que para $\delta>0$, existe $\epsilon, 0<\epsilon<1$, tal que

$$
n^{-1-\epsilon} \sum_{i=1}^{n} E_{\boldsymbol{\theta}}\left\{\sup _{\mathbf{h}:\|\mathbf{h}\| \leq \delta}\left\|\frac{\partial \boldsymbol{\psi}_{i}}{\partial \boldsymbol{\theta}}(\boldsymbol{\theta}+\mathbf{h})-\frac{\partial \boldsymbol{\psi}_{i}}{\partial \boldsymbol{\theta}}(\boldsymbol{\theta})-\boldsymbol{\phi}_{\delta_{\imath}}\right\|\right\}^{1+\epsilon} \rightarrow \mathbf{0}
$$

f. que para $\dot{\psi}_{i}(\theta)=\frac{\partial \psi_{i}}{\partial \theta^{\top}}(\theta)$.

$$
\frac{1}{n} \sum_{i=1}^{n} E\left\{\dot{\psi}_{i}(\theta)\right\}=\frac{1}{n} \sum_{i=1}^{n} \mathbf{S}_{i}(\theta) \rightarrow \mathbf{S}(\theta) .
$$

com $\mathbf{S}$ não singular e $\mathbf{S}_{i}=E\left\{\dot{\boldsymbol{\psi}}_{i}(\boldsymbol{\theta})\right\}$; 
g. que existe $\epsilon, 0<\epsilon<1$, tal que

$$
n^{-1-\epsilon} \sum_{i=1}^{n} E\left|\dot{\psi}_{i}(\theta)-\mathbf{S}_{i}(\theta)\right|^{1+\epsilon} \rightarrow 0
$$

h. que existe $\epsilon, 0<\epsilon<1$, tal que $\max _{1 \leq i \leq n} \max _{1 \leq j \leq t} E\left|\psi_{i j}\right|^{2+\epsilon}<\infty$;

i. $n^{-1} \sum_{i=1}^{n} \operatorname{Cov}\left(\psi_{i}\right) \rightarrow \mathbf{V}(\boldsymbol{\theta})$, positiva definida;

j. $\hat{\boldsymbol{\theta}}_{n}$ como a soluçấo de $\mathbf{\Psi}(\mathbf{w})=\mathbf{0}, \mathbf{w} \in \Theta$

e sob condiçôes que garantam a existência de uma sequência de raízes de $\mathbf{\Psi}_{n}(\mathbf{w})$ que seja limitada em probabilidade, ou restrita a um conjunto compacto q.c. quando $n$ tende a infinito ${ }^{3}$, vem que $\hat{\boldsymbol{\theta}}_{n} \stackrel{\mathcal{P}}{\rightarrow} \boldsymbol{\theta}$.

Corolário 2 . Sob as hipóteses do Teorema 3

$$
\sqrt{n}\left(\hat{\boldsymbol{\theta}}_{n}-\boldsymbol{\theta}\right) \stackrel{\mathcal{D}}{\rightarrow} \mathcal{N}_{p}\left\{\mathbf{0} ; \mathbf{J}_{\Psi}^{-1}(\boldsymbol{\theta})\right\}
$$

onde $J_{\Psi}(\boldsymbol{\theta})=\mathbf{S}(\boldsymbol{\theta}) \mathbf{V}^{-1}(\boldsymbol{\theta}) \mathbf{S}^{\top}(\boldsymbol{\theta})$.

As demonstrações dos Teoremas 2 e 3 e dos Corolários 1 e 2 são similares às provas da normalidade assintótica do estimador de máxima verossimilhança descritas em Sen e Singer (1993) e baseiam-se na propriedade de diferenciabilidade quadrática, desenvolvida por LeCam (1956).

No caso das distribuições marginais de $\mathrm{y}_{i}$ serem conhecidas, mas a conjunta não, as hipóteses (a), (b) e (d) do Teorema 2 são facilmente verificáveis. Problemas com a hipótese (c) relacionam-se à validade das condições (iv) e (v) da Definição 2, sendo necessária alguma informação sobre a distribuição conjunta do vetor. A hipótese (e) pode ser facilmente verificada se as componentes $\frac{\partial \psi_{i}}{\partial \theta}$ são limitadas e contínuas nas vizinhanças de $\theta$.

Em termos das hipóteses do Teorema 3, comentários análogos podem ser feitos sobre os itens (a) a (e). A validade do itern (h) depende apenas das distribuições marginais dos dados. A hipótese (i) é de difícil verificaçào se a distribuição conjunta dos dados é completamente desconhecida.

O Teorema 4 é uma versão alternativa do Teorema 3.

\footnotetext{
${ }^{3}$ na Seção 2.2.1, são explicitadas algumas condições que garantem esses resultados
} 
Teorema 4. Considerando-se

a. $\mathbf{y}_{i}, i=1,2, \ldots, n$, vetores aleatórios $t$-dimensionais independentes;

b. $\boldsymbol{\psi}_{i}(\boldsymbol{\theta})=\left(\boldsymbol{\psi}_{i 1}, \boldsymbol{\psi}_{i 2}, \ldots, \boldsymbol{\psi}_{i p}\right)^{\top}, i=1,2, \ldots, n$, funções de estimação regulares;

c. $\Psi_{n}(\theta)=\sum_{i=1}^{n} \psi_{i}(\theta)$;

d. $\operatorname{para} \delta>0$,

$$
\frac{1}{n} \sum_{i=1}^{n} \sup _{\mathbf{h}:\|\mathbf{h}\| \leq \delta}\left\|\frac{\partial \psi_{i}}{\partial \theta}(\theta+\mathbf{h})-\frac{\partial \psi_{i}}{\partial \boldsymbol{\theta}}(\boldsymbol{\theta})\right\| \stackrel{\mathcal{P}}{\rightarrow} \phi_{\delta},
$$

conforme $n \rightarrow \infty$, com $\phi_{\delta} \rightarrow 0$ quando $\delta \rightarrow 0$;

e. que quando $n \rightarrow \infty$,

$$
\frac{1}{n} \dot{\Psi}_{n}(\theta) \stackrel{\mathcal{P}}{\rightarrow} \mathbf{S}(\theta)
$$

f. $n^{-1} \sum_{i=1}^{n} \operatorname{Cov}\left(\psi_{i}\right) \rightarrow \mathbf{V}(\theta)$, positiva definida;

g. que quando $n \rightarrow \infty$,

$$
\frac{1}{\sqrt{n}} \boldsymbol{\Psi}_{n}(\boldsymbol{\theta}) \stackrel{\mathcal{D}}{\rightarrow} \mathcal{N}_{p}\{\mathbf{0} ; \mathrm{V}(\boldsymbol{\theta})\}
$$

h. $\hat{\boldsymbol{\theta}}_{n}$ como a solução de $\boldsymbol{\Psi}(\mathrm{w})=0, \mathrm{w} \in \Theta$

e sob condiçôes que garantam a existência de uma sequência de raízes de $\mathbf{\Psi}_{n}(\mathbf{w})$ que seja limitada em probabilidade, ou restrita a um conjunto compacto q.c. quando $n$ tende a infinito ${ }^{4}$, vem que $\hat{\boldsymbol{\theta}}_{n} \stackrel{\mathcal{P}}{\rightarrow} \boldsymbol{\theta}$.

Nessa nova versão do Teorema 3, as hipóteses (d), (e) e (f), vêm substituir, respectivamente, as hipóteses $(\mathrm{d}, \mathrm{e}),(\mathrm{f}, \mathrm{g})$ e $(\mathrm{h})$ daquele teorema. As hipóteses $(\mathrm{d}, \mathrm{e})$ e $(\mathrm{f}, \mathrm{g})$ do Teorema 3 correspondem à condiçào de Markov aplicada, respectivamente, a $\sum_{i=1}^{n} \sup _{\mathbf{h}: \| \mathbf{h} \mid \leq \delta}\left\|\frac{\partial \boldsymbol{\psi}_{i}}{\partial \boldsymbol{\theta}}(\boldsymbol{\theta}+\mathrm{h})-\frac{\partial \boldsymbol{\psi}_{i}}{\partial \boldsymbol{\theta}}(\theta)\right\|$ e $\dot{\boldsymbol{\Psi}}_{n}$ : a hipótese (h). por sua vez, é a condição de Liapunov, aplicada a $\boldsymbol{\Psi}_{n}$ (veja mais detalhes sobre essas condições em Sen e Singer, 1993, por exemplo).

\footnotetext{
${ }^{4}$ na Seção 2.2.1, são explicitadas algumas condições que garantem esses resultados
} 


\subsubsection{Complemento}

Nesta seção são apresentados alguns resultados relativos às condições de regularidade mencionadas nos Teoremas 2, 3 e 4. A Proposição 1 foi apresentada em Huber (1967) e Huber (1981), sendo que sua demonstração encontra-se em Huber (1967); já a Proposição 2 é demonstrada em Crowder (1986). Outros resultados a esse respeito podem ser encontrados em Inagaki (1973) e LeCam (1956), por exemplo.

Proposição 1 . (Huber, 1967). Seja $\Theta$ um espaço localmente compacto com uma base contável e $\psi(y, \theta)$ uma função de estimação assumindo valores $\mathrm{em}$ $\Re^{p}$. Considere $\mathrm{y}_{i}, i=1,2, \ldots, n$, uma amostra independente e identicamente distribuída de vetores aleatórios. Então se existir uma função contínua positiva, $b(\theta) \geq b_{0}>0$, tal que

a. $\sup _{\boldsymbol{\theta} \in \Theta} \frac{\left\|\boldsymbol{\psi}\left(y_{1}, \boldsymbol{\theta}\right)\right\|}{b(\boldsymbol{\theta})}$ seja integrável,

b. $\liminf _{\|\boldsymbol{\theta}\| \rightarrow \infty} \frac{\left\|E_{0}\left\{\boldsymbol{\psi}\left(y_{1}, \boldsymbol{\theta}\right)\right\}\right\|}{b(\boldsymbol{\theta})} \geq 1$, onde $E_{0}$ indica que a esperança deve ser tomada considerando-se a medida de probabilidade $P_{0}=P_{\theta_{0}}$,

c. $E_{0}\left[\limsup _{\|\boldsymbol{\theta}\| \rightarrow \infty} \frac{\left\|\boldsymbol{\psi}\left(y_{1}, \boldsymbol{\theta}\right)-E_{0}\left\{\boldsymbol{\psi}\left(y_{1}, \boldsymbol{\theta}\right)\right\}\right\|}{b(\boldsymbol{\theta})}\right]<1$,

então existe um conjunto compacto $C \subset \Theta$ tal que qualquer sequência $T_{n}$ satisfazendo

$$
\frac{1}{n} \sum_{i=1}^{n} \boldsymbol{\psi}\left(y_{i}, T_{n}\right) \stackrel{\text { q.c. }}{\rightarrow} \mathbf{0}
$$

quando $n$ tende a infinito, permanecerá em $C$.

Note que se $\left\{T_{n}\right\}$ é definida como uma sequência de raízes de $\sum_{i=1}^{n} \psi\left(y_{i}, \theta\right)$ a condição (2.3) está obviamente satisfeita.

Proposição 2 . (Crowder, 1986). Seja $\boldsymbol{\Psi}_{n}(\boldsymbol{\theta})$ uma função de estimaçâo $e$ $m_{n \boldsymbol{\theta}}=\left\|E_{0}\left\{\boldsymbol{\Psi}_{n}(\boldsymbol{\theta})\right\}\right\|$, onde $E_{0}$ é como na Proposição 1. Defina

$$
\begin{array}{r}
\overline{\boldsymbol{\Psi}}_{n}(\boldsymbol{\theta})=\boldsymbol{\Psi}_{n}(\boldsymbol{\theta})-E_{0}\left\{\boldsymbol{\Psi}_{n}(\boldsymbol{\theta})\right\} \\
\text { e } R_{n m}=\left\{\boldsymbol{\theta} \in \Theta: m_{n} \boldsymbol{\theta} \leq m, m>0\right\} \text {, entâo }
\end{array}
$$


A. se $\Theta$ for compacto $e$

$$
\sup _{\Theta-R_{n m}}\left\|\bar{\Psi}_{n}(\theta)\right\| \stackrel{\mathcal{P}}{\rightarrow} 0,
$$

onde a convergência se dá sob $P_{0}{ }^{5}$, então para cada $m>0$

$$
P_{0}\left\{R_{n m} \text { contém todas as raízes de } \boldsymbol{\Psi}_{n}(\boldsymbol{\theta})\right\} \rightarrow 1 \text {, }
$$

quando $n$ tende a infinito;

B. se $\Theta$ for não limitado, suponha que dado $\delta>0$ exista um conjunto compacto $C_{\delta}$ contendo todos os conjuntos $R_{n m}$ tais que

a. $\sup _{C_{\boldsymbol{\delta}}-R_{n m}}\left\|\overline{\boldsymbol{\Psi}}_{n}(\boldsymbol{\theta})\right\| \stackrel{\mathcal{P}}{\rightarrow} 0$, quando $n$ tende a infinito, sendo a convergência sob $P_{0} e$

b. $P_{0}\left\{\inf _{C_{\delta}-R_{n m}}\left\|\Psi_{n}(\boldsymbol{\theta})\right\|=0\right\}<\delta$,

para $n>N_{\delta}$, então a conclusão de (A) é válida.

\subsubsection{Aplicação}

Como exemplo de aplicação de resultados apresentados nesta seção, mais especificamente do Teorema 3 e da Proposição 2, considere uma amostra de $n$ variáveis aleatórias circulares, $y_{1}, y_{2}, \ldots, y_{n}$, e seja $\mu_{i}$ a média circular conhecida de $y_{i}$, com $E\left\{\cos \left(y_{i}-\mu_{i}\right)\right\}=\mathcal{A}_{1}(\lambda)$. Deseja-se demonstrar que, sob essas condiçôes o estimador de $\lambda$ obtido a partir de (1.19) é consistente e assintoticamente normal.

A partir de (1.19) pode-se definir a função de estimação

$$
\Phi_{n}(\lambda)=\mathcal{A}_{1}(\lambda)-\sum_{i=1}^{n} \frac{\cos \left(y_{i}-\mu_{i}\right)}{n} .
$$

A raiz dessa função é o estimador procurado. Note que a função de estimação $\Phi_{n}$ equivale, em termos de suas raízes, a

$$
\Psi_{n}(\lambda)=\sum_{i=1}^{n} \psi_{i}(\lambda)
$$

$\operatorname{com} \psi_{i}(\lambda)=\mathcal{A}_{1}(\lambda)-\cos \left(y_{i}-\mu_{i}\right)$.

\footnotetext{
${ }^{5}$ ver definição na Proposição 1
} 


\section{Regularidade da função de estimação}

Considere a função de estimação $\Psi_{n}$. A condição $(i)$ da Definição 2 é consequência imediata da suposição sobre a esperança de $\cos \left(y_{i}-\mu_{i}\right)$. Note que $\frac{\mathrm{d} \psi_{i}}{\mathrm{~d} \lambda}$ existe quase certamente, uma vez que $\mathcal{A}_{1}(\lambda)$ é diferenciável (ver Mardia, 1972, por exemplo).

Como

$$
\begin{aligned}
0 \leq \psi_{i}^{2}(\lambda) & =\mathcal{A}_{1}^{2}(\lambda)+\cos ^{2}\left(y_{i}-\mu_{i}\right)-2 \mathcal{A}_{1}(\lambda) \cos \left(y_{i}-\mu_{i}\right) \\
& \leq \mathcal{A}_{1}^{2}(\lambda)+\cos ^{2}\left(y_{i}-\mu_{i}\right)+2 \mathcal{A}_{1}(\lambda)\left|\cos \left(y_{i}-\mu_{i}\right)\right| \leq 4,
\end{aligned}
$$

a hipótese (iv) da Definição 2 é satisfeita.

Por fim,

$$
E\left\{\frac{\mathrm{d} \psi_{n}}{\mathrm{~d} \lambda}(\lambda)\right\}=1-\frac{\mathcal{A}_{1}(\lambda)}{\lambda}-\mathcal{A}_{1}^{2}(\lambda) \in \Re_{+}-0,
$$

o que garante a validade da condição (v) da Definição.

\section{Raízes da função de estimação}

Em termos de raízes, $\Psi_{n}$ equivale a $\Phi_{n}$. Por facilidade, será demonstrado que $\Phi_{n}$ satisfaz à Proposição 2 e o resultado pode então ser diretamente estendido a $\Psi_{n}$

Note que

$$
m_{n \lambda}=\left|E_{0}\left\{\Phi_{n}(\lambda)\right\}\right|=\left|\mathcal{A}_{1}(\lambda)-\mathcal{A}_{1}\left(\lambda_{0}\right)\right| .
$$

Logo, como $0<\mathcal{A}_{1}(\lambda)<1$, vem que

$$
\begin{aligned}
R_{n m} & =\left\{\lambda>0: m_{n \lambda} \leq m, m>0\right\} \\
& =\left[\mathcal{A}_{1}^{-1}\left[\left\{\mathcal{A}_{1}\left(\lambda_{0}\right)-m\right\} \mathbf{1}_{\left\{\mathcal{A}_{1}\left(\lambda_{0}\right)-m>0\right\}}\right],\right. \\
& \left.\mathcal{A}_{1}^{-1}\left[\left\{\mathcal{A}_{1}\left(\lambda_{0}\right)+m\right\} \mathbf{1}_{\left\{\mathcal{A}_{1}\left(\lambda_{0}\right)+m<1\right\}}+\mathbf{1}_{\left\{\mathcal{A}_{1}\left(\lambda_{0}\right)+m \geq 1\right\}}\right]\right],
\end{aligned}
$$

onde $\mathbf{1}_{A}$ é a função indicadora do conjunto $A$ e $\mathcal{A}_{1}^{-1}(1)=\propto$. Note que os intervalos $R_{n m}$ contêm $\lambda_{0}$. Por outro lado,

$$
\bar{\Phi}_{n}(\lambda)=\mathcal{A}_{1}\left(\lambda_{0}\right)-\sum_{i=1}^{n} \frac{\cos \left(y_{i}-\mu_{i}\right)}{n},
$$


e, sob hipóteses gerais ${ }^{6}$, pode-se mostrar que $\sum_{i=1}^{n} \frac{\cos \left(y_{i}-\mu_{i}\right)}{n}$ satisfaz a lei forte dos grandes números, convergindo para $\mathcal{A}_{1}\left(\lambda_{0}\right)$ quando $n$ tende a infinito. Isso faz com que $\left|\bar{\Phi}_{n}(\lambda)\right|$ convirja quase certamente para zero quando $n$ cresce e portanto a condição (a) da Proposição é satisfeita independentemente da escolha de $C_{\delta}$.

A verificação da condição (b) vem do fato de $\left|\Phi_{n}(\lambda)\right|$ convergir quase certamente para $\left|\mathcal{A}_{1}(\lambda)-\mathcal{A}_{1}\left(\lambda_{0}\right)\right|$ que difere de zero se $\lambda \neq \lambda_{0}$, pois $\mathcal{A}_{1}(\lambda)$ é uma função crescente. Logo, dados $m$ e $\delta$ existe $N_{\delta}$ suficientemente grande, tal que

$$
P_{0}\left\{\inf _{C_{\delta}-R_{n m}}\left\|\Psi_{n}(\boldsymbol{\theta})\right\|=0\right\}<\delta,
$$

uma vez que $\lambda_{0}$ não pertence a $C_{\delta}-R_{n m}$. Esses resultados garantem que as raízes de $\Phi_{n}$ (e consequentemente de $\Psi_{n}$ ) estarão quase certamente restritas a um intervalo limitado, quando $n$ cresce.

\section{As outras hipóteses}

A seguir verificaremos que $\Psi_{n}$ satisfaz as outras hipóteses do Teorema 3 .

Como

$$
\frac{\mathrm{d} \Psi_{n}}{\mathrm{~d} \lambda}(\lambda)=1-\frac{\mathcal{A}_{1}(\lambda)}{\lambda}-\mathcal{A}_{1}^{2}(\lambda)
$$

supondo $|h| \leq \delta$, vem que

$$
\begin{aligned}
& \left|\frac{\mathrm{d} \Psi_{n}}{\mathrm{~d} \lambda}(\lambda+h)-\frac{\mathrm{d} \Psi_{n}}{\mathrm{~d} \lambda}(\lambda)\right| \\
& =\left|\frac{\mathcal{A}_{1}(\lambda)}{\lambda}+\mathcal{A}_{1}^{2}(\lambda)-\frac{\mathcal{A}_{1}(\lambda+h)}{\lambda+h}-\mathcal{A}_{1}^{2}(\lambda+h)\right| \\
& \leq\left|\frac{\mathcal{A}_{1}(\lambda)}{\lambda}-\frac{\mathcal{A}_{1}(\lambda+h)}{\lambda+h}\right|+\left|\mathcal{A}_{1}^{2}(\lambda)-\mathcal{A}_{1}^{2}(\lambda+h)\right| \\
& \leq\left|\frac{\lambda\left\{\mathcal{A}_{1}(\lambda)-\mathcal{A}_{1}(\lambda+h)\right\}+h \mathcal{A}_{1}(\lambda)}{\lambda^{2}}\right|+\left|\mathcal{A}_{1}^{2}(\lambda)-\mathcal{A}_{1}^{2}(\lambda+h)\right| \\
& \leq \frac{1}{\lambda}\left|\mathcal{A}_{1}(\lambda)-\mathcal{A}_{1}(\lambda+\delta)\right|+\delta \frac{\mathcal{A}_{1}(\lambda)}{\lambda^{2}}+\mathcal{A}_{1}^{2}(\lambda+h)-\mathcal{A}_{1}^{2}(\lambda)=T(\delta)
\end{aligned}
$$

\footnotetext{
${ }^{6}$ basta supor, por exemplo, que a distribuição de $y_{i}$ depende apenas de $\mu_{i}$ e de $\lambda$ e que a distribuição $y_{i}-\mu_{i}$ não depende de $i, i=1,2, \ldots, n$
} 
e consequentemente

$$
E_{\theta}\left\{\sup _{h:|h| \leq \delta}\left|\frac{\mathrm{d} \Psi_{n}}{\mathrm{~d} \lambda}(\lambda+h)-\frac{\mathrm{d} \Psi_{n}}{\mathrm{~d} \lambda}(\lambda)\right|\right\}=\phi_{\delta i} \leq T(\delta) .
$$

Segue então

$$
\frac{1}{n} \sum_{i=1}^{n} \phi_{\delta i} \leq T(\delta) \rightarrow 0
$$

quando $\delta$ converge para zero, o que verifica a hipótese (d) do Teorema 3 .

Quanto à hipótese (e), note que para $h$ satisfazendo $|h| \leq \delta$, vem que

$$
\left|\frac{\mathrm{d} \Psi_{n}}{\mathrm{~d} \lambda}(\lambda+h)-\frac{\mathrm{d} \Psi_{n}}{\mathrm{~d} \lambda}(\lambda)-\phi_{\delta i}\right| \leq 2 T(\delta)
$$

e então

$$
\begin{aligned}
n^{-1-\epsilon} \sum_{i=1}^{n} E_{\theta}\left\{\sup _{h:|h| \leq \delta}\left|\frac{\mathrm{d} \Psi_{n}}{\mathrm{~d} \lambda}(\lambda+h)-\frac{\mathrm{d} \Psi_{n}}{\mathrm{~d} \lambda}(\lambda)-\phi_{\delta i}\right|\right\}^{1+\epsilon} & \leq n^{-\epsilon}\{2 T(\delta)\}^{1+\epsilon} \\
& \rightarrow 0
\end{aligned}
$$

quando $n$ tende a infinito, para qualquer $\epsilon>0$.

Para verificar as hipóteses (f) e $(\mathrm{g})$, basta notar que $E\left\{\dot{\psi}_{i}(\lambda)\right\}$ é uma constante que independe de $i$.

A hipótese (h) é uma consequência do fato de $\left|\Psi_{i}\right| \leq 2$, uma vez que $\mathcal{A}_{1}(\lambda) \leq 1$.

Para verificar a hipótese (i), basta notar que a parte aleatória de $\Psi_{i}$ depende apenas de $\cos \left(y_{i}-\mu_{i}\right)$ e que

$$
\operatorname{Var}\left\{\cos \left(y_{i}-\mu_{i}\right)\right\}=E\left\{\cos ^{2}\left(y_{i}-\mu_{i}\right)\right\}-E^{2}\left\{\cos \left(y_{i}-\mu_{i}\right)\right\} \leq 1-\mathcal{A}_{1}^{2}(\lambda) \text {. }
$$

\subsubsection{Demonstrações}

Para facilitar a demonstração do Teorema 2, considere o seguinte lema:

Lema 1 . Sob as hipóteses (a), (b), (c) e (d) do Teorema \& tem-se que

$$
\frac{1}{\sqrt{n}} \Psi_{n}(\theta) \stackrel{\mathcal{D}}{\rightarrow} \mathcal{N}\{0 ; \mathrm{V}(\theta)\}
$$

Prova. O resultado segue da aplicação direta do teorema limite central a $\Psi_{n}$. 


\section{Prova do Teorema 2}

Seja $\mathbf{u} \in \Re^{p}$ tal que $\theta+n^{-1 / 2} \mathbf{u} \in \Theta$. Então, expandindo $\Psi_{n}\left(\theta+n^{-1 / 2} \mathbf{u}\right)$ por série de Taylor ao redor de $\theta\left(u_{0}=0\right)$, vem que

$$
\boldsymbol{\Psi}_{n}\left(\boldsymbol{\theta}+n^{-1 / 2} \mathbf{u}\right)=\boldsymbol{\Psi}_{n}(\boldsymbol{\theta})+\frac{1}{\sqrt{n}} \dot{\Psi}_{n}\left(\boldsymbol{\theta}^{*}\right) \mathbf{u},
$$

onde $\theta^{*}$ pertence ao segmento de reta que une $\theta$ e $\theta+n^{-1 / 2} \mathbf{u}$ e $\dot{\Psi}_{n}(\theta)=$ $\frac{\partial \Psi_{n}}{\partial \boldsymbol{\theta}^{T}}(\boldsymbol{\theta})$. Dividindo ambos os membros por $\sqrt{n}$, vem

$$
\frac{1}{\sqrt{n}} \Psi_{n}\left(\boldsymbol{\theta}+n^{-1 / 2} \mathbf{u}\right)=\frac{1}{\sqrt{n}} \boldsymbol{\Psi}_{n}(\boldsymbol{\theta})+\frac{1}{n} \dot{\Psi}_{n}\left(\boldsymbol{\theta}^{*}\right) \mathbf{u}
$$

Seja

$$
\frac{1}{n} \dot{\Psi}_{n}\left(\theta^{*}\right)=\mathbf{E}_{n}+\mathbf{Z}_{n}
$$

onde

$$
\mathbf{E}_{n}=\frac{1}{n} \dot{\Psi}_{n}(\theta)
$$

e

$$
\mathbf{Z}_{n}(\mathbf{u})=\frac{1}{n} \dot{\boldsymbol{\Psi}}_{n}\left(\boldsymbol{\theta}^{*}\right)-\mathbf{E}_{n} .
$$

De $(2.8)$ e (2.5), vem

$$
\frac{1}{\sqrt{n}} \Psi_{n}\left(\boldsymbol{\theta}+n^{-1 / 2} \mathbf{u}\right)=\frac{1}{\sqrt{n}} \Psi_{n}(\boldsymbol{\theta})+\mathbf{E}_{n} \mathbf{u}+\mathbf{Z}_{n}(\mathrm{u}) \mathrm{u}
$$

Note que:

I. Dado $K>0$, para $\|\mathbf{u}\| \leq K$ e dado $\delta>0$, existe $n_{0}=n_{0}(\delta, K)$ tal que $n^{-1 / 2}\|\mathbf{u}\| \leq n^{-1 / 2} K<\delta, \forall n \geq n_{0}$. Então para $n$ suficientemente grande, com $\dot{\psi}_{i}=\frac{\bar{\partial} \psi_{i}}{\partial \boldsymbol{\theta}^{\top}}$, vem

$$
\begin{aligned}
\left\|\mathbf{Z}_{n}(\mathbf{u})\right\| & =\frac{1}{n}\left\|\sum_{i=1}^{n}\left\{\dot{\boldsymbol{\psi}}_{i}\left(\theta^{*}\right)-\dot{\psi}_{i}(\boldsymbol{\theta})\right\}\right\| \\
& \leq \frac{1}{n} \sum_{i=1}^{n} \sup _{\left\{\mathrm{h}:\|\mathrm{h}\| \leq n^{-1 / 2}\|\mathbf{u}\|\right\}}\left\|\dot{\boldsymbol{\psi}}_{i}(\boldsymbol{\theta}+\mathrm{h})-\dot{\boldsymbol{\psi}}_{i}(\boldsymbol{\theta})\right\| \\
& \leq \frac{1}{n} \sum_{i=1}^{n} \sup _{\{\mathrm{h}:\|\mathrm{h}\| \leq \delta\}}\left\|\dot{\psi}_{i}(\boldsymbol{\theta}+\mathrm{h})-\dot{\boldsymbol{\psi}}_{i}(\boldsymbol{\theta})\right\| .
\end{aligned}
$$


Considerando (a), (b) e (e), vem, pela lei forte dos grandes números de Khintchine, que

$$
\frac{1}{n} \sum_{i=1}^{n} \sup _{\{\mathbf{h}:\|\mathbf{h}\| \leq \delta\}}\left\|\dot{\psi}_{i}(\theta+\mathbf{h})-\dot{\psi}_{i}(\theta)\right\| \stackrel{\text { q.c. }}{\rightarrow} \phi_{\delta} .
$$

Como $\phi_{\delta} \rightarrow 0$ quando $\delta \rightarrow 0$ e a escolha de $\delta$ é arbitrária, vem que

$$
\sup _{\{\mathbf{u}:\|\mathbf{u}\| \leq K\}}\left\|\mathbf{Z}_{n}(\mathbf{u})\right\| \stackrel{\text { q.c. }}{\rightarrow} \mathbf{0}
$$

uniformemente quando $n$ cresce e para $K$ arbitrário.

II. De (a), (b), (c) e (d) vem, pela lei fraca dos grandes números de Khintchine aplicada a cada elemento da matriz $\mathbf{E}_{n}(\theta)$, que

$$
\mathbf{E}_{n}=\frac{1}{n} \dot{\Psi}_{n}(\theta)=\frac{1}{n} \sum_{i=1}^{n} \dot{\psi}_{i}(\theta) \stackrel{\mathcal{P}}{\rightarrow} \mathbf{S}(\theta),
$$

quando $n$ tende a infinito.

Pode-se reescrever (2.9) como

$$
\frac{1}{\sqrt{n}} \boldsymbol{\Psi}_{n}\left(\boldsymbol{\theta}+n^{-1 / 2} \mathbf{u}\right)=\frac{1}{\sqrt{n}} \boldsymbol{\Psi}_{n}(\boldsymbol{\theta})+\mathbf{S}(\boldsymbol{\theta}) \mathbf{u}+\left\{\mathbf{E}_{n}-\mathbf{S}(\boldsymbol{\theta})\right\} \mathbf{u}+\mathbf{Z}_{n}(\mathbf{u}) \mathbf{u}
$$

Aplicando (2.11) e (2.12), para $\mathbf{u}$ fixo, tem-se, uniformemente para $\|\mathbf{u}\| \leq K$, que

$$
\frac{1}{\sqrt{n}} \boldsymbol{\Psi}_{n}\left(\boldsymbol{\theta}+n^{-1 / 2} \mathbf{u}\right)=\frac{1}{\sqrt{n}} \boldsymbol{\Psi}_{n}(\boldsymbol{\theta})+\mathbf{S}(\boldsymbol{\theta}) \mathbf{u}+o_{p}(1) .
$$

Temos que demonstrar que o resultado (2.13) mantém-se verdadeiro quando $\mathbf{u}$ é substituído por $\hat{\mathbf{u}}_{n}$, onde $\boldsymbol{\theta}+n^{-1 / 2} \hat{\mathbf{u}}_{n}$ corresponde à raiz de $\boldsymbol{\Psi}_{n}(\boldsymbol{\theta})$. Por hipótese, existe uma sequência $\left\{\hat{\mathbf{u}}_{n}\right\}$ que se mantém restrita a um conjunto compacto e, nesse caso, a conjectura é verdadeira, ou existe uma sequência $\left\{\hat{\mathbf{u}}_{n}\right\}$ limitada em probabilidade e nesse caso, é necessária a utilização do Item (III) abaixo.

III. Suponha que $\left\{\hat{\mathbf{u}}_{n}^{*}\right\}$, seja uma sequência, limitada em probabilidade, tal que $\theta+\hat{\mathrm{u}}_{n}^{*} / \sqrt{n} \in \Theta$. Mostraremos que

$$
\left\|\mathrm{Z}_{n}\left(\hat{\mathbf{u}}_{n}^{*}\right)\right\| \stackrel{\mathcal{P}}{\rightarrow} 0
$$


quando $n$ tende a infinito. Tomando qualquer $\delta>0$, então para qualquer $\epsilon>0$, tem-se

$$
\begin{aligned}
P\left\{\left\|\mathbf{Z}_{n}\left(\hat{\mathbf{u}}_{n}^{*}\right)\right\|>\epsilon\right\} & =P\left\{\left\|\mathbf{Z}_{n}\left(\hat{\mathbf{u}}_{n}^{*}\right)\right\|>\epsilon ; n^{-1 / 2} \hat{\mathbf{u}}_{n}^{*} \leq \delta\right\} \\
& +P\left\{\left\|\mathbf{Z}_{n}\left(\hat{\mathbf{u}}_{n}^{*}\right)\right\|>\epsilon ; n^{-1 / 2} \hat{\mathbf{u}}_{n}^{*}>\delta\right\} \\
& \leq P\left\{\left\|\mathbf{Z}_{n}\left(\hat{\mathbf{u}}_{n}^{*}\right)\right\|>\epsilon \mid n^{-1 / 2} \hat{\mathbf{u}}_{n}^{*} \leq \delta\right\} \\
& +P\left\{n^{-1 / 2} \hat{\mathbf{u}}_{n}^{*}>\delta\right\} .
\end{aligned}
$$

Uma vez que $\hat{\mathbf{u}}_{n}^{*}$ é limitada em probabilidade, para qualquer $\delta>0$

$$
P\left\{n^{-1 / 2} \hat{\mathbf{u}}_{n}^{*}>\delta\right\} \rightarrow 0,
$$

conforme $n$ tende a infinito. Por outro lado, aplicando-se I, vem que

$$
P\left\{\left\|\mathbf{Z}_{n}\left(\hat{\mathbf{u}}_{n}^{*}\right)\right\|>\epsilon \mid n^{-1 / 2} \hat{\mathbf{u}}_{n}^{*} \leq \delta\right\} \rightarrow 0
$$

conforme $n$ tende a infinito e $\delta$, que foi tomado arbitrariamente, tende a zero. Portanto, conclui-se que

$$
\mathbf{Z}_{n}\left(\hat{\mathbf{u}}_{n}^{*}\right) \stackrel{\mathcal{P}}{\rightarrow} \mathbf{0} .
$$

IV. Retornando a (2.13), considerando-se $\hat{\mathbf{u}}_{n}$ uma raiz de $\boldsymbol{\Psi}_{n}\left(\boldsymbol{\theta}+n^{-1 / 2} \mathbf{u}\right)$, vem, sob a hipótese de que a sequência de raízes é limitada em probabilidade, ou que para $n$ suficientemente grande está restrita a um conjunto compacto, que

$$
\hat{\mathrm{u}}=-\mathbf{S}^{-1}(\boldsymbol{\theta})\left\{\frac{1}{\sqrt{n}} \boldsymbol{\Psi}_{n}(\boldsymbol{\theta})\right\}+o_{p}(1) .
$$

Além disso, denominando de $\hat{\theta}_{n}$ a raiz de $\boldsymbol{\Psi}_{n}(\mathbf{w})$, vem que

$$
\hat{\boldsymbol{\theta}}_{n}=\boldsymbol{\theta}+n^{-1 / 2} \hat{\mathbf{u}}_{n}=\boldsymbol{\theta}-\mathbf{S}^{-1}(\boldsymbol{\theta})\left\{\frac{1}{n} \boldsymbol{\Psi}_{n}(\boldsymbol{\theta})\right\}+o_{p}\left(\frac{1}{\sqrt{n}}\right) .
$$

Considerando $\frac{1}{n} \Psi_{n}(\theta)=\frac{1}{n} \sum_{i=1}^{n} \psi_{i}(\theta)$, das hipóteses (a). (b), (c) e (d), vem, pela lei fraca dos grandes números de Khintchine aplicada a cada elemento do vetor $\frac{1}{n} \Psi_{n}(\theta)$ que

$$
\frac{1}{n} \boldsymbol{\Psi}_{n}(\boldsymbol{\theta}) \stackrel{\mathcal{P}}{\rightarrow} 0 .
$$


Substituindo-se (2.15) em (2.14) e aplicando-se o teorema de Slutsky, vem que

$$
\hat{\boldsymbol{\theta}}_{n}=\theta+o_{p}(1)
$$

o que conclui a prova. $\circ$

\section{Prova do Corolário 1:}

De (2.14) vem que

$$
\sqrt{n}\left(\hat{\boldsymbol{\theta}}_{n}-\boldsymbol{\theta}\right)=-\mathbf{S}^{-1}(\boldsymbol{\theta})\left\{\frac{1}{\sqrt{n}} \boldsymbol{\Psi}_{n}(\boldsymbol{\theta})\right\}+o_{p}(1) ;
$$

o Lema 1 garante que

$$
\frac{1}{\sqrt{n}} \Psi_{n}(\theta) \stackrel{\mathcal{D}}{\rightarrow} \mathcal{N}\{0 ; \mathrm{V}(\boldsymbol{\theta})\}
$$

onde $\mathbf{V}(\boldsymbol{\theta})=E\left\{\boldsymbol{\psi}_{1}(\boldsymbol{\theta}) \boldsymbol{\psi}_{1}^{\top}(\boldsymbol{\theta})\right\}$

Aplicando-se (2.17) em (2.16), e utilizando-se o teorema de Slutsky, vem que

$$
\sqrt{n}\left(\hat{\boldsymbol{\theta}}_{n}-\boldsymbol{\theta}\right) \stackrel{\mathcal{D}}{\rightarrow} \mathcal{N}_{p}\left\{\mathbf{0} ; \mathbf{S}^{-1}(\boldsymbol{\theta}) \mathrm{V}(\boldsymbol{\theta}) \mathbf{S}^{-\top}(\boldsymbol{\theta})\right\},
$$

o que conclui a prova.o dos.

Para facilitar a demonstração do Teorema 3, dois lemas serão apresenta-

Lema 2 . Sob as hipóteses (a), (b), (c), (h) e (i) do Teorema 3, vem que

$$
\frac{1}{\sqrt{n}} \psi_{n}(\theta) \stackrel{\mathcal{D}}{\rightarrow} \mathcal{N}_{p}\{\mathbf{0} ; \mathbf{V}(\theta)\}
$$

$e \frac{1}{n} \Psi_{n}(\boldsymbol{\theta}) \stackrel{\mathcal{P}}{\rightarrow} 0$ quando $n \rightarrow \infty$.

Prova: Aplicando-se as hipóteses (h) e (i) em $\frac{1}{\sqrt{n}} \psi_{n}(\theta)$, o teorema limite central de Liapunov garante (2.18). A convergência em probabilidade de $\frac{1}{n} \Psi_{n}(\theta)$ é uma consequência.o

Note que para provar a convergência em probabilidade acima, pode-se substituir as hipóteses (h) e (i) por condições mais simples, tal como a condição de Markov (ver em Sen e Singer, 1993), por exemplo. 
Lema 3 . Sob as hipóteses (a), (b), (c), (f) e (g) do Teorema 3, vem que

$$
\frac{1}{n} \sum_{i=1}^{n} \dot{\Psi}_{n}(\theta) \stackrel{\mathcal{P}}{\rightarrow} \mathbf{S}(\theta)
$$

quando $n \rightarrow \infty$.

Prova: As hipóteses (f) e (g) permitem a aplicação da lei fraca dos grandes números de Markov a cada componente do vetor $\frac{1}{n} \sum_{i=1}^{n} \dot{\Psi}_{n}(\boldsymbol{\theta})$ e consequentemente segue-se o resultado.o

\section{Prova do Teorema 3:}

A prova é semelhante à do Teorema 2: diferenças aparecern nas demonstrações das convergências de (2.15), (2.10) e (2.12).

Ás condições (d) e (e) permitem a aplicação da lei fraca dos grandes números de Markov, então

$$
\frac{1}{n} \sum_{i=1}^{n} \sup _{\{\mathbf{h}:\|\mathbf{h}\| \leq \delta\}}\left\|\dot{\psi}_{i}(\boldsymbol{\theta}+\mathbf{h})-\dot{\psi}_{i}(\boldsymbol{\theta})\right\| \stackrel{\mathcal{P}}{\rightarrow} \phi_{\delta} .
$$

Dos Lemas 2, 3 e do resultado acima, a prova é obtida seguindo-se os passos da demonstração do Teorema 2. o

\section{Prova do Corolário 2:}

A prova é análoga à do Corolário 1 , basta utilizar a relação (2.16) e o resultado (2.18). o 


\section{Capítulo 3}

\section{Funções de estimação para modelos de dispersão longitudinais}

Neste capítulo são sugeridas equações de estimação adequadas à estimação de parâmetros marginais de um modelo com estrutura longitudinal. Tais equações fornecem estimadores consistentes e assintoticamente normais desses parâmetros.

Suponha que o elemento $i$ de uma amostra aleatória, $i=1,2, \ldots, n$, seja observado $t_{i}$ vezes ao longo de alguma dimensão, por exemplo o tempo, e que cada observação corresponda a uma realização de uma variável aleatória $y_{i j}$. Além disso, admita que cada observação sofra a influência de um conjunto de $p$-covariáveis, sendo $\mathrm{x}_{i j}=\left(x_{i j 1}, x_{i j 2}, \ldots, x_{i j p}\right)^{\top}$ o vetor que contém os valores de tais variáveis para o indivíduo $i$, observação $j$. Defina um vetor $t_{i} \times 1 \mathrm{com}$ as respostas observadas para o indivíduo $i, \mathbf{y}_{i}=\left(y_{1 i}, \ldots, y_{i t_{1}}\right)^{\top}$ e associado a esse, uma matriz $t_{i} \times p, \mathbf{X}_{i}=\left(\mathbf{x}_{i 1}, \ldots, \mathbf{x}_{i t_{i}}\right)^{\top}$. Finalmente, admita que $y_{i j} \sim \mathcal{M D}\left(\mu_{i j} ; \sigma^{2} / w_{i j}\right), i=1,2, \ldots, n$ e $j=1,2, \ldots, t_{i}$, onde $w_{i j}$ são pesos positivos e defina $\boldsymbol{\mu}_{i}=\left(\mu_{i 1}, \mu_{i 2}, \ldots, \mu_{i t_{i}}\right)^{\mathrm{T}}$.

$\mathrm{Na}$ Seção 3.1 é discutida a modelagem e estimação do parâmetro de posição; já na Seção 3.3 , tanto o parâmetro de posiçâo como o de dispersão são modelados conjuntamente.

Para facilitar a notação, assuma, sem perda de generalidade, que $t_{i}=t$, $i=1,2, \ldots, n$. 


\subsection{Modelagem do parâmetro de posição}

O primeiro passo é o estabelecimento de um modelo para o parâmetro de posição. Seja $g: \mathcal{R} \rightarrow \mathcal{R}$ uma função inversível, duplamente diferenciável e tal que

$$
g\left(\mu_{i j}\right)=\sum_{k=1}^{p} x_{i j k} \beta_{k}=\eta_{i j}
$$

onde $\boldsymbol{\beta}=\left(\beta_{1}, \ldots, \beta_{p}\right)^{\top}$ é um vetor paramétrico real.

Uma primeira análise pode ser conduzida ignorando-se a estrutura de dependência existente entre as observações de uma mesma unidade amostral. Nesse caso, a função escore de $\boldsymbol{\beta}$ é proporcional a

$$
\bar{\Psi}_{n}^{I}(\boldsymbol{\beta})=\sum_{i=1}^{n} \psi_{i}^{I}(\boldsymbol{\beta})
$$

onde

$$
\psi_{i}^{I}(\boldsymbol{\beta})=\mathbf{D}_{i}^{\top} \mathbf{u}_{i}
$$

$\mathbf{D}_{i}^{\top}=\mathbf{X}_{i}^{\top} \mathbf{H}_{i} \mathbf{A}_{i}, \mathbf{H}_{i}=\frac{\partial \mu_{i}}{\partial \eta_{i}}=\operatorname{diag}\left\{\frac{\partial \mu_{i 1}}{\partial \eta_{i 1}}, \ldots, \frac{\partial \mu_{i t}}{\partial \eta_{i t}}\right\}, \mathbf{u}_{i}$ é um vetor $t$-dimensional com componentes $u_{i j}=\frac{\partial d}{\partial \mu_{i j}}\left(y_{i j} ; \mu_{i j}\right)$ e $\mathbf{A}_{i}=\operatorname{diag}\left\{w_{i 1}, \ldots, w_{i t}\right\}$.

Seja $\hat{\boldsymbol{\beta}}_{n}^{I}$ a raiz da função de estimação $\boldsymbol{\Psi}_{n}^{I}$. Sob condições gerais de regularidade (ver o Teorema 3 , por exemplo), pode-se demonstrar que essa raiz é um estimador consistente de $\boldsymbol{\beta}$ e, mais ainda, que

$$
\sqrt{n}\left(\hat{\boldsymbol{\beta}}_{n}^{I}-\boldsymbol{\beta}\right) \stackrel{\mathcal{D}}{\rightarrow} \mathcal{N}_{p}\left(0, \overline{\mathbf{J}}^{I^{-1}}\right)
$$

onde

com

$$
\overline{\mathbf{J}}^{I}=\lim _{n \rightarrow \infty} n\left\{\sum_{i=1}^{n} \mathbf{S}_{i}^{I}\right\}\left\{\sum_{i=1}^{n} \mathbf{V}_{i}^{I}\right\}^{-1}\left\{\sum_{i=1}^{n} \mathbf{S}_{i}^{I}\right\},
$$

$$
\begin{aligned}
& \mathbf{V}_{i}^{I}=\mathbf{D}_{i}^{\top} \operatorname{Cov}\left(\mathbf{u}_{i}\right) \mathbf{D}_{i}, \\
& \mathbf{S}_{i}^{I}=\mathbf{X}_{i}^{\top} \mathbf{H}_{i} \mathbf{A}_{i} E\left\{\Delta\left(\dot{\mathbf{u}}_{i}\right)\right\} \mathbf{H}_{i} \mathbf{X}_{i} \mathrm{e} \\
& \dot{\mathbf{u}}_{i}=\left(\frac{\partial u_{i 1}}{\partial \mu_{i 1}}, \frac{\partial u_{i 2}}{\partial \mu_{i 2}}, \ldots, \frac{\partial u_{i t}}{\partial \mu_{i t}}\right)^{\top} .
\end{aligned}
$$


$\overline{\mathbf{J}}^{I^{-1}}$ pode ser consistentemente estimado por

$$
\hat{\overline{\mathbf{J}}}_{I}^{-1}=n\left\{\sum_{i=1}^{n} \mathbf{S}_{i}^{I}\right\}^{-1}\left\{\mathbf{D}_{i}^{\top} \mathbf{u}_{i}^{\top} \mathbf{u}_{i} \mathbf{D}_{i}\right\}\left\{\sum_{i=1}^{n} \mathbf{S}_{i}\right\}^{-\top},
$$

sendo todas as quantidades avaliadas em $\hat{\boldsymbol{\beta}}_{n}^{I}$. Na literatura especializada esse estimador recebe o nome de "estimador sanduíche".

Apesar do estimador obtido a partir das raízes de (3.1) possuir propriedades assintóticas interessantes, a função de estimação não leva em conta o fato de existir uma estrutura de dependência entre os elementos de $\mathbf{y}_{i}$. $\mathrm{Na}$ verdade, o estimador $\hat{\boldsymbol{\beta}}_{n}^{I}$ pode ser bastante ineficiente no caso de amostras pequenas.

\subsection{1 $\operatorname{Cov}\left(\mathbf{u}_{i}\right)$ conhecida}

Nesta seção serão introduzidas modificações em $\Psi_{n}^{I}$ de modo que a nova função de estimação incorpore alguma informação sobre a estrutura de dependência dos dados.

Note que $\Psi_{n}^{I}$ pertence à classe $\mathcal{L}$, tendo como funções geradoras da classe $\mathbf{u}_{i}, i=1,2, \ldots, n$. Assim, podemos obter um estimador melhorado de $\boldsymbol{\beta}$ se, ao invés de $\Psi_{n}^{I}$, utilizarmos a função de estimação ótima na classe $\mathcal{L}$; tal função é dada por $\Psi_{n}^{*}(\boldsymbol{\beta})=\sum_{i=1}^{n} \boldsymbol{\psi}_{i}^{*}(\boldsymbol{\beta})$, onde

$$
\dot{\psi}_{i}^{*}(\boldsymbol{\beta})=\mathbf{D}_{i}^{\top} \mathbf{N}_{i} \mathbf{W}_{i}^{-1} \mathbf{u}_{i}=\mathbf{X}_{i}^{\top} \mathbf{H}_{i} \mathbf{A}_{i} \mathbf{N}_{i} \mathbf{W}_{i}^{-1} \mathbf{u}_{i},
$$

$\operatorname{com} \mathbf{W}_{i}=\operatorname{Cov}\left(\mathbf{u}_{i}\right)$ e $\mathbf{N}_{i}=\Delta\left\{E\left(\dot{\mathbf{u}}_{i}\right)\right\}, \operatorname{com} \dot{\mathbf{u}}_{i}=\left(\dot{u}_{i 1} \ldots, \dot{u}_{i t}\right)^{\top}, \dot{u}_{i j}=$ $\partial u_{i j} / \partial \mu_{i j}$. Defina $\mathbf{D}_{i}^{*}=\mathbf{D}_{i} \mathbf{N}_{i}$.

É fácil ver que se $d$ e $g^{-1}$ são diferenciáveis até segunda ordem e se $\boldsymbol{\psi}_{i}^{*}$ satisfaz as condições (iv) e (v) da Definiçào 2, ela é regular e sua respectiva matriz de informação de Godambe de $\boldsymbol{\beta}$ é dada por

$$
J_{\psi_{i}^{*}}(\boldsymbol{\beta})=\mathrm{S}_{i}^{\top} \mathbf{V}_{i}^{*-1} \mathbf{S}_{i}
$$

onde

$$
\begin{aligned}
\mathbf{V}_{i}^{*} & =\mathbf{D}_{i}^{* \top} \mathbf{W}_{i}^{-1} \operatorname{Cov}\left(\mathrm{u}_{i}\right) \mathbf{W}_{i}^{-1} \mathbf{D}_{i}^{*}=\mathbf{D}_{i}^{* \top} \mathbf{W}_{i}^{-1} \mathbf{D}_{i}^{*} \mathrm{e} \\
\mathbf{S}_{i} & =\mathbf{D}_{i}^{* \top} \mathbf{W}_{i}^{-1} \mathbf{D}_{i}^{*} .
\end{aligned}
$$


Considere a função de estimação dada por (3.3) e seja $\hat{\boldsymbol{\beta}}_{n}$ a solução de $\boldsymbol{\Psi}_{n}^{*}(\boldsymbol{\beta})=\mathbf{0}$. Sob as condições do Teorema 3 , vem que $\hat{\boldsymbol{\beta}}_{n}$ é um estimador consistente de $\boldsymbol{\beta}$ e

$$
\sqrt{n}\left(\hat{\boldsymbol{\beta}}_{n}-\boldsymbol{\beta}\right) \stackrel{\mathcal{D}}{\rightarrow} \mathcal{N}_{p}\left[\mathbf{0}, \lim _{n \rightarrow \infty} n\left\{\sum_{i=1}^{n} \mathbf{S}_{i}\right\}^{-1} \sum_{i=1}^{n} \mathbf{V}_{i}\left\{\sum_{i=1}^{n} \mathbf{S}_{i}\right\}^{-\mathrm{T}}\right] .
$$

A prova desse resultado é uma consequência do Teorema 3 e do Corolário 2 .

A normalidade assintótica pode ser obtida sob condições mais brandas quando $y_{i j} \sim \mathcal{M D}\left(\mu_{j}, \sigma^{2} / w_{j}\right)$. Esse é o caso onde a variável resposta é observada sob diferentes situações para cada elemento da amostra, sendo que a única mudança esperada diz respeito ao parâmetro de locação entre as medidas de um mesmo indivíduo. Nesse caso particular, pode-se considerar o seguinte resultado:

Teorema 5. Sejam $y_{i j} \sim \mathcal{M D}\left(\mu_{j} ; \sigma^{2} / w_{j}\right)$ e a função de estimação dada por

$$
\boldsymbol{\Psi}_{n}(\boldsymbol{\beta})=\sum_{i=1}^{n} \mathbf{D}^{* \top} \mathbf{W}^{-1} \mathbf{u}_{i}
$$

Então sob as condições do Teorema 2 vem que $\hat{\boldsymbol{\beta}}_{n} \stackrel{\mathcal{P}}{\rightarrow} \boldsymbol{\beta} e$

$$
\sqrt{n}\left(\hat{\boldsymbol{\beta}}_{n}-\boldsymbol{\beta}\right) \stackrel{\mathcal{D}}{\rightarrow} \mathcal{N}_{p}\left\{\mathbf{0}, \mathbf{S}^{-1} \mathbf{V S}^{-\top}\right\}
$$

quando $n \rightarrow \infty$, onde

$$
\mathbf{S}=\mathbf{D}^{* \top} \mathbf{W}^{-1} \mathbf{D}^{*} \text { e } \mathbf{V}=\mathbf{D}^{* \top} \mathbf{W}^{-1} \operatorname{Cov}\left(u_{1}\right) \mathbf{W}^{-1} \mathbf{D}^{*}
$$

Prova: consequência do Teorema 2 e Corolário 1.0

\subsection{2 $\operatorname{Cov}\left(\mathbf{u}_{i}\right)$ desconhecida}

Em termos práticos a função $\boldsymbol{\Psi}_{n}^{*}$ tem pouca importância, uma vez que, em geral, as matrizes $\operatorname{Cov}\left(\mathbf{u}_{i}\right)$ são desconhecidas. Isto pode ser contornado, substituindo-se $\mathbf{W}_{i}$ por um estimador, mas assim fazendo, a nova função de estimação será provavelmente viciada, o que pode prejudicar a qualidade do estimador de $\boldsymbol{\beta}$ obtido, em termos de suas propriedades assintóticas. A metodologia desenvolvida a seguir é uma generalização do trabalho de Liang e Zeger (1986) a uma classe mais ampla de modelos. 
Considere a existência de um vetor paramétrico $\alpha$ que define a matriz de covariâncias de $\mathbf{u}_{i}, \mathbf{W}_{i}=\mathbf{W}_{i}(\boldsymbol{\alpha}), i=1,2, \ldots, n$. Dessa forma, o problema reduz-se a encontrar um estimador de $\alpha, \hat{\boldsymbol{\alpha}}_{n}$, de tal modo que o estimador obtido a partir de

$$
\Psi_{n}(\boldsymbol{\beta})=\sum_{i=1}^{n} \mathbf{D}_{i}^{* \top} \mathbf{W}_{i}^{-1}\left(\hat{\alpha}_{n}\right) \mathbf{u}_{i}
$$

continue sendo consistente e assintoticamente normal. O Teorema 6 descreve algumas condições que o estimador de $\alpha$ deve satisfazer de modo que o estimador de $\boldsymbol{\beta}$ preserve tais propriedades.

Teorema 6 . Seja $\hat{\boldsymbol{\beta}}_{n}$ a raiz de (3.7), sob condições gerais de regularidade ${ }^{1}$, com $\left\|\hat{\boldsymbol{\beta}}_{n}-\boldsymbol{\beta}\right\|=O_{p}(1)$ e assumindo que

a. $\hat{\boldsymbol{\alpha}}_{n}\left(\boldsymbol{\beta}, \sigma^{2}\right)$ é um estimador $\sqrt{n}$-consistente ${ }^{2}$ de $\boldsymbol{\alpha}$ dados $\boldsymbol{\beta}$ e $\sigma^{2}$;

b. $\hat{\sigma}_{n}^{2}=\hat{\sigma}_{n}^{2}(\beta)$ é um estimador $\sqrt{n}$-consistente de $\sigma^{2}$, dado $\boldsymbol{\beta}$;

c. $\left|\frac{\partial \hat{\boldsymbol{\alpha}}_{n}\left(\boldsymbol{\beta}, \sigma^{2}\right)}{\partial \sigma^{2}}\right| \leq \mathbf{H}(\mathbf{y}, \boldsymbol{\beta})$, onde $\mathbf{H}(\mathbf{y}, \boldsymbol{\beta})$ é uma função $O_{p}(1)$ de $\boldsymbol{\beta}$ e dos tem-se que $\hat{\boldsymbol{\beta}}_{n}$ é um estimador consistente de $\boldsymbol{\beta} e$

$$
n^{1 / 2}\left(\hat{\boldsymbol{\beta}}_{n}-\boldsymbol{\beta}\right) \stackrel{\mathcal{D}}{\rightarrow} \mathcal{N}\left(\mathbf{0}, \overline{\mathbf{J}}^{-1}\right)
$$

quando $n \rightarrow \infty$, onde

$$
\overline{\mathbf{J}}^{-1}=\lim _{n \rightarrow \infty} n\left\{\sum_{i=1}^{n} \mathbf{S}_{i}\right\}^{-1}\left\{\sum_{i=1}^{n} \mathbf{V}_{i}\right\}\left\{\sum_{i=1}^{n} \mathbf{S}_{i}\right\}^{-\boldsymbol{T}},
$$

onde $\mathbf{S}_{i}$ é dada em (3.5) e $\mathbf{V}_{i}=\mathbf{D}_{i}^{* \top} \mathbf{W}_{i}^{-1} \operatorname{Cov}\left(\mathbf{u}_{i}\right) \mathbf{W}_{i}^{-1} \mathbf{D}_{i}^{*}$.

Prova: a prova desse resultado é similar à descrita em Liang e Zeger (1986) e encontra-se na Seção 3.1.7.

A função de estimação da técnica "GEE" de Liang e Zeger (1986) é um caso particular de (3.7), como será visto na Seção 3.2.1

\footnotetext{
${ }^{1}$ veja Seção 3.1.7

${ }^{2}$ isto é, $\left\|\alpha_{n}\left(\beta, \sigma^{2}\right)-\alpha\right\|=O_{p}\left(n^{-1 / 2}\right)$.
} 
Note que os resultados assintóticos do Teorema 6 são válidos mesmo quando $\mathbf{W}(\boldsymbol{\alpha})$ não corresponde à verdadeira matriz de covariâncias de $\mathbf{u}_{i}$ e suas hipóteses exigem apenas que o estimador de $\alpha$ satisfaça às hipóteses do Teorema 6 . A especificação correta dessa matriz, contudo, pode levar a estimadores mais eficientes (ver Liang et al., 1992, Fitzmaurice at al.. 1993 e Albert e McShane, 1995, onde considera-se a técnica "GEE").

$\overline{\mathbf{J}}^{-1}$ pode ser consistentemente estimada pelo "estimador sanduíche"

$$
\hat{\overline{\mathbf{J}}}^{-1}=n\left(\sum_{i=1}^{n} \mathbf{S}_{i}\right)^{-1}\left(\mathbf{D}_{i}^{* \top} \mathbf{W}_{i}^{-1} \mathbf{u}_{i}^{\top} \mathbf{u}_{i} \mathbf{W}_{i}^{-1} \mathbf{D}_{i}^{*}\right)\left(\sum_{i=1}^{n} \mathbf{S}_{i}\right)^{-\mathrm{T}},
$$

onde todas as quantidades são avaliadas no ponto $\hat{\boldsymbol{\beta}}$. Quando $\mathbf{W}_{i}$ corresponde a uma estimativa consistente da verdadeira matriz de covariâncias de $\mathbf{u}_{i}, \overline{\mathbf{J}}^{-1}$ pode ser consistentemente estimada por

$$
\hat{\overline{\mathbf{J}}}^{-1}=n\left\{\sum_{i=1}^{n} \mathbf{S}_{i}\right\}^{-1} .
$$

Firth (1992) destaca que essa estimativa é mais eficiente do que a apresentada anteriormente. Prentice (1988) chega a uma conclusão semelhante ao estudar sua aplicação aos estimadores obtidos a partir das "GEE".

\subsubsection{Estimação de $\sigma^{2}$}

Nesta seção propomos dois métodos para a estimação do parâmetro de dispersão. Ambos conduzem a estimadores que satisfazem às condições do Teorema 6 .

\section{Estimador baseado na verossimilhança marginal}

Sob a hipótese de independência entre as observações de um mesmo indivíduo e dado $\boldsymbol{\beta}$, a função escore do parâmetro $\sigma^{2}$ é

$$
\Phi_{n}\left(\sigma^{2}\right)=\sum_{i=1}^{n} \sum_{j=1}^{t}\left\{\frac{\dot{a}_{i j}}{w_{i j} a\left(y_{i j}, \sigma^{2} / w_{i j}\right)}+\frac{w_{i j}}{2 \sigma^{4}} d\left(y_{i j}, \mu_{i j}\right)\right\} .
$$

onde $\dot{a}_{i j}=\left.\frac{\partial a}{\partial x}\left(y_{i j}, x\right)\right|_{x=\sigma^{2} / w_{i j}}$.

É fácil ver que $\Phi_{n}\left(\sigma^{2}\right)$ é uma função de estimação regular. desde que satisfaça às condições (iv) and (v) da Definição 2. 
Seja $\hat{\sigma}^{2}$ a solução de $\Phi_{n}\left(\sigma^{2}\right)=0$. Se $\Phi_{n}\left(\sigma^{2}\right)$ for uma função de estimação regular, sob as hipóteses do Teorema 3 e dado $\boldsymbol{\beta}$ conhecido, ela será um estimador consistente de $\sigma^{2} \mathrm{e}$

$$
\sqrt{n}\left(\hat{\sigma}^{2}-\sigma^{2}\right) \stackrel{\mathcal{D}}{\rightarrow} \mathcal{N}\left\{0, J_{\Phi_{n}}^{-1}\left(\sigma^{2}\right)\right\}
$$

onde

$$
J_{\Phi_{n}}\left(\sigma^{2}\right)=\frac{E^{2}\left\{\dot{\Phi}_{n}\left(\sigma^{2}\right)\right\}}{E\left\{\Phi_{n}^{2}\left(\sigma^{2}\right)\right\}}
$$

e

$$
\dot{\Phi}_{n}\left(\sigma^{2}\right)=\frac{d \Phi_{n}\left(\sigma^{2}\right)}{d \sigma^{2}}=\sum_{i=1}^{n} \sum_{j=1}^{t} \frac{\ddot{a}_{i j} a\left(y_{i j}, \sigma^{2}\right)-\left(\dot{a}_{i j}\right)^{2}}{w_{i j}^{2} a^{2}\left(y_{i j}, \sigma^{2}\right)}-\sum_{i=1}^{n} \sum_{j=1}^{t} \frac{w_{i j}}{\sigma^{6}} d\left(y_{i j}, \mu_{i j}\right),
$$

$\operatorname{com} \ddot{a}_{i j}=\left.\frac{\partial \dot{a}_{i j}}{\partial x}\right|_{x=\sigma^{2} / w_{i j}}$.

\section{Estimador de Pearson}

Com base em (1.21), pode-se definir um estimador consistente para $\sigma^{2}$ dado pela solução de

$$
\hat{\sigma}^{2}=\frac{1}{n t-p} \sum_{i=1}^{n} X^{2}\left(\hat{\boldsymbol{\mu}}_{i}, \sigma^{2}\right)=\frac{1}{n t-p} \sum_{i=1}^{n} \sum_{j=1}^{t} V\left(\mu_{i j} ; \sigma^{2}\right) \tilde{u}^{2}\left(y_{i} ; \mu_{i j}\right) .
$$

\subsubsection{Estimação de W}

Supondo-se $\mathbf{W}=\operatorname{Cov}\left(\mathbf{u}_{i}\right)$, um estimador $\sqrt{n}$-consistente de $\mathbf{W}$ pode ser obtido através de

ou mesmo,

$$
\hat{\mathbf{W}}=\frac{\sum_{i=1}^{n} \mathbf{u}_{i} \mathbf{u}_{i}^{\top}}{n}
$$

$$
\hat{\mathbf{W}}=\frac{\sum_{i=1}^{n}\left(\mathbf{u}_{i}-\overline{\mathbf{u}}\right)\left(\mathbf{u}_{i}-\overline{\mathbf{u}}\right)^{\top}}{n},
$$

onde $\overline{\mathbf{u}}$ é o vetor média aritmética de $\mathbf{u}_{i}, i=1,2, \ldots, n$, sendo que $\mathbf{u}_{i}$ deve ser avaliada a cada passo do procedimento de estimação, conforme salientado na Seção 3.1.6. 
Assuma $\mathbf{W}_{i}=\operatorname{Cov}\left(\mathbf{u}_{i}\right)$ e considere a seguinte decomposição:

$$
\mathbf{W}_{i}=\operatorname{Var}^{1 / 2}\left(\mathbf{u}_{i}\right) \mathbf{R}_{i}(\boldsymbol{\alpha}) \operatorname{Var}^{1 / 2}\left(\mathbf{u}_{i}\right)
$$

onde $\mathbf{R}_{i}(\boldsymbol{\alpha})$ corresponde à matriz de correlaçâo de $\mathbf{u}_{i}$ e $\operatorname{Var}\left(\mathbf{u}_{i}\right)$ a uma matriz diagonal, cujo $j$-ésimo elemento da diagonal principal corresponde a $\operatorname{Var}\left(u_{i j}\right)$. Serão apresentadas sugestões para a estimação $\sqrt{n}$-consistente de $\alpha$ em alguns casos onde $\mathbf{R}_{i}(\boldsymbol{\alpha})=\mathbf{R}(\boldsymbol{\alpha}), i=1, \ldots, n$ e em que $\operatorname{Var}\left(u_{i j}\right)$ não depende de $i$ e $j^{3}$

Considere, inicialmente, que não se tenha nenhuma informação sobre a estrutura da matriz de correlação. Assim, $\boldsymbol{\alpha}$ será um vetor com $t(t-1) / 2$ componentes, onde $\alpha_{j k}, j$ diferente de $k$, corresponde à correlação entre $u_{i j} \mathrm{e}$ $u_{i k}, i=1, \ldots, n$ e $j, k=1, \ldots, t$. Um estimador natural para $\alpha_{j k}$ é

$$
\hat{\alpha}_{j k}=\frac{\sum_{i=1}^{n} u_{i j} u_{i k}}{\left(\sum_{i=1}^{n} u_{i j}^{2}\right)^{1 / 2}} \frac{\left(\sum_{i=1}^{n} u_{i k}^{2}\right)^{1 / 2}}{}
$$

Eventualmente, pode-se supor que os dados satisfaçam alguma estrutura de correlação. Isso faz com que o número de parâmetros de perturbação que necessitam ser estimados diminua. Uma estrutura bastante conhecida é a uniforme e nesse caso a correlação entre as observações de dois instantes em um mesmo indivíduo é sempre a mesma. Tem-se então $\alpha=\alpha$. Um possível estimador para essa quantidade é

$$
\hat{\alpha}=\frac{\sum_{i=1}^{n} \sum_{j>k} u_{i j} u_{i k}}{\left(\sum_{i=1}^{n} \sum_{j=1}^{t} u_{i j}^{2}\right)} \frac{n t}{\frac{n t(t-1)}{2}}=\frac{\sum_{i=1}^{n} \sum_{j>k} u_{i j} u_{i k}}{\left(\sum_{i=1}^{n} \sum_{j=1}^{t} u_{i j}^{2}\right)} \frac{2}{t-1} .
$$

Outra estrutura bastante estudada é a auto-regressiva de ordem 1. Nesse

\footnotetext{
${ }^{3} \mathrm{Na}$ Seção 3.2.1 são apresentados estimadores alternativos quando se considera modelos exponenciais de dispersão.
} 
caso, $\alpha=\alpha$ e Corr $\left(u_{i j}, u_{i k}\right)=\alpha^{|j-k|}$. Um estimador simples para $\alpha$ é

$$
\hat{\alpha}=\frac{\sum_{i=1}^{n} \sum_{j=1}^{t-1} u_{i j} u_{i, j+1}}{\left(\sum_{i=1}^{n} \sum_{j=1}^{t-1} u_{i j}^{2} \sum_{i=1}^{n} \sum_{j=2}^{t} u_{i j}^{2}\right)^{1 / 2}} .
$$

Uma deficiência desse estimador é que ele utiliza somente a informação sobre a dependência de observações consecutivas de $u_{i j}$. Estimadores alternativos que levam em conta uma quantidade maior de informação podem ser obtidos, observando-se que

$$
E\left(u_{i j} u_{i k}\right)=\left\{\operatorname{Var}\left(u_{i j}\right) \operatorname{Var}\left(u_{i k}\right)\right\}^{1 / 2} \alpha^{|j-k|} .
$$

Desse modo, por mínimos quadrados, pode-se ajustar um modelo de regressão tendo a forma acima, $\operatorname{com}\left(u_{i j} u_{i k}\right)$ como variável dependente e $|j-k|$ como independente. Alternativamente, pode-se ajustar um modelo de regressão linear com $\log \left(u_{i j} u_{i k}\right)$ como variável dependente, $\log |j-k|$ como independente e o estimador de $\alpha$ é dado pelo estimador do coeficiente angular da regressão.

\section{Estimação de $\operatorname{Cov}\left(\mathbf{u}_{i}\right)$ via equações de estimação}

Uma abordagem alternativa para a estimação de $\mathbf{W}_{i}$ é adicionar novas equações de estimação a $\boldsymbol{\Psi}_{n}$, que sejam responsáveis pela estimação de $\boldsymbol{\alpha}$. Seja $\boldsymbol{\theta}=\left(\boldsymbol{\beta}^{\top}, \boldsymbol{\alpha}^{\top}\right)^{\top}$ e $\sigma_{i j k}\left(\boldsymbol{\theta}, \sigma^{2}\right)$ a covariância entre as observações dos instantes $j$ e $k$ para o indivíduo $i, i=1,2, \ldots, n$ e $j, k=1,2, \ldots, t$. Sabe-se que

$$
E\left(u_{i j} u_{i k}\right)=\sigma_{i j k}\left(\theta, \sigma^{2}\right)=\sigma_{i j k}, i=1, \ldots, n, \text { e } j, k=1, \ldots, t .
$$

Considere, por exemplo,

$\mathbf{v}_{i}=\left(u_{i 1}^{2}-\sigma_{i 11}, u_{i 1} u_{i 2}-\sigma_{i 12}, \ldots, u_{i 1} u_{i t}-\sigma_{i 1 t}, \ldots, u_{i, t-1} u_{i t}-\sigma_{i, t-1, t}, u_{i t}^{2}-\sigma_{i t t}\right)^{\top}$.

É fácil ver que $E\left(\mathbf{v}_{i}\right)=0, i=1,2, \ldots, n$. Logo, uma equação de estimaçáo ótima para $\theta$ na classe $\mathcal{L}$ é dada por

$$
\Psi_{n}^{*}(\boldsymbol{\theta})=\sum_{i=1}^{n} E\left[\begin{array}{cc}
\mathbf{D}_{i}^{* \top} & \mathbf{0} \\
\frac{\partial \mathbf{v}_{i}}{\partial \boldsymbol{\beta}} & \frac{\partial \mathbf{v}_{i}}{\partial \boldsymbol{\alpha}}
\end{array}\right]\left[\begin{array}{cc}
\operatorname{Cov}\left(\mathbf{u}_{i}\right) & \operatorname{Cov}\left(\mathbf{u}_{i}, \mathbf{v}_{i}\right) \\
\operatorname{Cov}\left(\mathbf{v}_{i}, \mathbf{u}_{i}\right) & \operatorname{Cov}\left(\mathbf{v}_{i}\right)
\end{array}\right]^{-1}\left[\begin{array}{c}
\mathbf{u}_{i} \\
\mathbf{v}_{i}
\end{array}\right]=0
$$


Um problema com o uso prático dessas equações é que novas quantidades desconhecidas, $\operatorname{Cov}\left(\mathbf{v}_{i}\right)$ e $\operatorname{Cov}\left(\mathbf{u}_{i}, \mathbf{v}_{i}\right)$, são introduzidas, o que requer novas suposições sobre suas estruturas (no caso de modelos exponenciais de dispersão, suposições sobre o terceiro e quarto momentos da distribuição do vetor respesta, por exemplo). Pode-se modelar esses valores através da introdução de um novo vetor paramétrico, exatamente como foi feito para a estimação de $\operatorname{Cov}\left(\mathbf{u}_{i}\right)$.

É possível demonstrar um resultado similar ao Teorema 6 para o novo estimador de $\boldsymbol{\theta}$ (veja um resultado análogo em Prentice, 1988 e Prentice e Zhao, 1991). A matriz de covariância assintótica das estimativas pode ser obtida da matriz de informação de Godambe das parcelas de (3.13), como foi feito em (3.6) e Teorema 6. O número de parâmetros a serem estimados pode ser reduzido se abrirmos mão da otimalidade das equações de estimação. Pode-se obter, por exemplo

$$
\Psi_{n}(\theta)=\sum_{i=1}^{n}\left[\begin{array}{cc}
\mathbf{D}_{i}^{* \top} & \mathbf{0} \\
\mathbf{0} & \frac{\partial \mathbf{v}_{i}}{\partial \alpha}
\end{array}\right]\left[\begin{array}{cc}
\operatorname{Cov}^{-1}\left(\mathbf{u}_{i}\right) & \mathbf{0} \\
\mathbf{0} & \operatorname{Cov}^{-1}\left(\mathbf{v}_{i}\right)
\end{array}\right]\left[\begin{array}{c}
\mathbf{u}_{i} \\
\mathbf{v}_{i}
\end{array}\right]=\mathbf{0}
$$

ou, eventualmente, até

$$
\Psi_{n}(\theta)=\sum_{i=1}^{n}\left[\begin{array}{cc}
\mathbf{D}_{i}^{* \top} & \mathbf{0} \\
\mathbf{0} & \frac{\partial \mathbf{v}_{i}}{\partial \alpha}
\end{array}\right]\left[\begin{array}{cc}
\operatorname{Cov}^{-1}\left(\mathbf{u}_{i}\right) & \mathbf{0} \\
\mathbf{0} & \mathbf{I}_{t}
\end{array}\right]\left[\begin{array}{c}
\mathbf{u}_{i} \\
\mathbf{v}_{i}
\end{array}\right]=\mathbf{0}
$$

onde $\mathbf{I}_{t}$ é uma matriz identidade $t$-dimensional. Assim agindo, afastamo-nos da função de estimação ótima em $\mathcal{L}$, mas limitamos a quantidade de suposições a serem feitas sobre a estrutura de dependência dos dados. Propriedades assintóticas das raízes de (3.14) e (3.15), similares às relatadas no Teorema 6, são asseguradas se as funções de inferência propostas satisfazem as hipóteses do Teorema 3. Um maior nível de simplicidade pode ser obtido ao substituir-se $\mathbf{D}_{i}^{*}$ por $\mathbf{D}_{i}$, sem prejuízo da consistência e normalidade assintótica do correspondente estimador.

Pode-se acrescentar $\Phi_{n}\left(\sigma^{2}\right)$ a essas equações para obter uma estimativa de $\sigma^{2}$.

Para a validade dos resultados assintóticos é necessário que $E\left(\mathrm{v}_{i}\right)=0$, $i=1,2, \ldots, n$; logo, impondo uma estrutura de correlação especial para $\mathbf{u}_{i}$, deve-se assegurar que ela é a correta, ou a hipótese de não vício da função de estimação poderá ser falsa e o correspondente estimador pode não possuir as propriedades mencionadas. 
Prentice (1988) e Prentice e Zhao (1991) estudaram equações de estimação análogas a (3.13) e (3.14), para a análise de modelos de dispersão exponenciais.

\subsubsection{Teste de hipóteses}

Considerando-se a distribuição assintótica de $\hat{\boldsymbol{\beta}}$ pode-se testar a hipótese linear geral, $\mathcal{H}: \mathbf{C} \boldsymbol{\beta}=\mathbf{0}$, onde $\mathbf{C}$ é uma matriz de posto completo $q \leq p$, utilizando-se a seguinte estatística de teste:

$$
Q=\hat{\boldsymbol{\beta}}_{n}^{\top} \mathbf{C}^{\top}\left\{\operatorname{CCov}_{A}\left(\hat{\boldsymbol{\beta}}_{n}\right) \mathbf{C}^{\top}\right\}^{-1} \mathbf{C} \hat{\boldsymbol{\beta}}_{n},
$$

onde $\operatorname{Cov}_{A}\left(\hat{\boldsymbol{\beta}}_{n}\right)$ é a matriz de covariâncias assintóticas de $\hat{\boldsymbol{\beta}}_{n}$. Sob a hipótese $\mathcal{H}, Q \stackrel{\mathcal{D}}{\rightarrow} \chi_{q}$, quando $n \rightarrow \infty$. Na prática, pode-se susbstituir $\operatorname{Cov}_{A}\left(\hat{\boldsymbol{\beta}}_{n}\right)$ por um estimador consistente derivado, por exemplo, de (3.8) ou (3.9), se for o caso. Carr e Chi (1992) e Rotnitzky e Jewell (1990) apresentam testes semelhantes no contexto das "GEE".

\subsubsection{Algoritmos de estimação}

Para a obtenção de estimativas dos parâmetros do modelo (3.7), três abordagens serão desenvolvidas: o método de Newton, o modificado de Newton (ver Jørgensen et al., 1995) e um outro baseado no modificado de Newton.

Pode-se verificar que

$$
\begin{aligned}
\frac{\partial \boldsymbol{\psi}_{i}}{\partial \boldsymbol{\beta}} & =\mathbf{X}_{i}^{\top} \dot{\mathbf{H}}_{i} \mathbf{A}_{i} \mathbf{N}_{i} \mathbf{W}_{i}^{-1^{*}} \mathbf{X}_{i} \\
& \left.+\mathbf{D}_{i}^{\top}\left\{\dot{N}_{\mathbf{W}}^{-1} \Delta\left(\mathbf{u}_{i}\right)+\mathbf{N}_{i} \mathbf{W}_{i}^{-1} \Delta\left(\dot{u}_{i}\right)\right) \mathbf{N}_{i}\right\} \mathbf{D}_{i}
\end{aligned}
$$

onde $\dot{\mathbf{H}}_{i}=\operatorname{diag}\left(\frac{\partial^{2} \mu_{i 1}}{\partial \eta_{i 1}^{2}}, \ldots, \frac{\partial^{2} \mu_{i t}}{\partial \eta_{i t}^{2}}\right), \dot{\mathbf{N}}_{i}=\Delta\left(\ddot{\mathbf{u}}_{i}\right), \ddot{\mathbf{u}}_{i}=\left(\ddot{u}_{i 1}, \ldots, \ddot{u}_{i t}\right)^{\top}, \ddot{u}_{i j}=$ $\partial \dot{u}_{i j} / \partial \mu_{i j}, \mathbf{W}_{i}^{-1^{*}}=\operatorname{diag}\left(\sum_{k=1}^{t} w_{i 1 k} u_{i k}, \ldots, \sum_{k=1}^{t} w_{i t k} u_{i k}\right)$ e $w_{i j k}$ é o elemento da linha $j$ e coluna $k$ de $\mathbf{W}_{i}^{-1}$.

Considerando-se o sobrescrito $l$ como indicador da estimativa do parâmetro no passo $l$ do processo iterativo, vem que o algoritmo de Newton para $\beta$ é dado por:

$$
\beta^{(l+1)}=\beta^{(l)}-\mathbf{S}_{e}^{-1}\left(\beta^{(l)}\right) \Psi_{n}\left(\beta^{(l)}\right)
$$


onde $\mathbf{S}_{e}=\sum_{i=1}^{n} \frac{\partial \dot{\psi}_{i}}{\partial \boldsymbol{\beta}}$

$\mathrm{O}$ algoritmo modificado de Newton é obtido substituindo-se $\mathrm{S}_{e}$ por sua esperança: $\mathbf{S}=\sum_{i=1}^{n} \mathbf{S}_{i}\left(\boldsymbol{\beta}^{(l)}\right)$, com $\mathbf{S}_{i}$ definida em (3.5).

Um algoritmo alternativo pode ser sugerido, a partir do modificado de Newton, substituindo-se $\mathbf{S}$ por $\sum_{i=1}^{n} \mathbf{X}_{i}^{\top} \mathbf{H}_{i} \Delta\left(\dot{\mathbf{u}}_{i}\right) \mathbf{W}_{i}^{-1} \Delta\left(\dot{\mathbf{u}}_{i}\right) \mathbf{H}_{i} \mathbf{X}_{i}$. Na verdade, substitui-se $E\left\{\Delta\left(\dot{\mathbf{u}}_{i}\right)\right\}$ por $\Delta\left(\dot{\mathbf{u}}_{i}\right)$.

Durante a obtenção das estimativas dos parâmetros, sugere-se que em cada passo do processo a matriz $\mathbf{W}_{i}$ seja atualizada, avaliando-a no valor de $\hat{\boldsymbol{\beta}}_{n}$ daquele passo.

Métodos de estimação análogos a esses podem ser desenvolvidos para obter estimativas, utilizando-se as outras funções de estimação descritas anteriormente.

\subsubsection{Demonstrações}

A fim de simplificar a demonstração do Teorema 6, alguns lemas serão introduzidos, onde são apresentadas algumas das possíveis condições de regularidade mencionadas no enunciado do teorema. Denomine as hipóteses do Teorema 6 de $\mathcal{H}$.

Lema 4. Sob $\mathcal{H}$ e considerando-se $\hat{\boldsymbol{\alpha}}_{n}=\hat{\boldsymbol{\alpha}}_{n}\left(\boldsymbol{\beta}, \hat{\sigma}_{n}^{2}\right)$, vem que

$$
\sqrt{n}\left(\hat{\boldsymbol{\alpha}}_{n}-\boldsymbol{\alpha}\right)=O_{p}(1)
$$

Prova:

$$
\sqrt{n}\left(\hat{\boldsymbol{\alpha}}_{n}-\boldsymbol{\alpha}\right)=\sqrt{n}\left\{\hat{\boldsymbol{\alpha}}_{n}\left(\boldsymbol{\beta}, \hat{\sigma}_{n}^{2}(\boldsymbol{\beta})\right)-\hat{\boldsymbol{\alpha}}_{n}\left(\boldsymbol{\beta}, \sigma^{2}\right)+\hat{\boldsymbol{\alpha}}_{n}\left(\boldsymbol{\beta}, \sigma^{2}\right)-\boldsymbol{\alpha}\right\}
$$

e, pela hipótese (a), $\sqrt{n}\left\{\hat{\alpha}_{n}\left(\boldsymbol{\beta}, \sigma^{2}\right)-\boldsymbol{\alpha}\right\}=O_{p}\left(n^{-1 / 2}\right)$. Por outro lado, expandindo $\hat{\alpha}_{n}\left(\boldsymbol{\beta}, \sigma^{2}\right)$ em série de Taylor, em uma vizinhança de $\hat{\sigma}_{n}^{2}(\boldsymbol{\beta})$ e utilizando-se a hipótese (b), vem que

$$
\hat{\boldsymbol{\alpha}}_{n}\left(\boldsymbol{\beta}, \sigma^{2}\right)=\hat{\boldsymbol{\alpha}}_{n}\left(\boldsymbol{\beta}, \hat{\sigma}_{n}^{2}\right)+\frac{\partial \hat{\boldsymbol{\alpha}}_{n}}{\partial \sigma^{2}}\left(\boldsymbol{\beta}, \hat{\sigma}_{n}^{2}\right)\left(\sigma^{2}-\hat{\sigma}_{n}^{2}\right)+O_{p}\left(n^{-1 / 2}\right) .
$$


Quando $n$ cresce e aplicando as hipóteses (b) e (c), vem que

$$
\hat{\boldsymbol{\alpha}}_{n}\left(\boldsymbol{\beta}, \hat{\sigma}_{n}^{2}(\boldsymbol{\beta})\right)-\hat{\boldsymbol{\alpha}}_{n}\left(\boldsymbol{\beta}, \sigma^{2}\right)=-\frac{\partial \hat{\boldsymbol{\alpha}}_{n}}{\partial \sigma^{2}}\left(\boldsymbol{\beta}, \hat{\sigma}_{n}^{2}\right)\left(\sigma^{2}-\hat{\sigma}_{n}^{2}\right)+O_{p}\left(n^{-1 / 2}\right) .
$$

Logo,

$$
\sqrt{n}\left(\hat{\boldsymbol{\alpha}}_{n}-\boldsymbol{\alpha}\right)=O_{p}(1) .
$$

Lema 5. Sob $\mathcal{H}$, assumindo que $\partial \Psi_{n}(\boldsymbol{\beta}, \boldsymbol{\alpha}) / \partial \boldsymbol{\alpha}$ satisfaz a condição de Markov $^{4}$ e $\max _{1 \leq i \leq n} \max _{i<i \leq n} E\left|\Psi_{i j}\right|^{2+\delta}<\infty$ para algum $0<\delta<1$, onde $\Psi_{i j}$ é a j-ésima componente do vetor $\boldsymbol{\Psi}_{i}$, então

$$
\frac{1}{\sqrt{n}} \Psi_{n}\left(\boldsymbol{\beta}, \hat{\boldsymbol{\alpha}}_{n}\right) \stackrel{\mathcal{D}}{\rightarrow} \mathcal{N}_{p}(\mathbf{0}, \mathbf{V})
$$

quando $n \rightarrow \infty$, onde $\mathbf{V}=\lim _{n \rightarrow \infty} \sum_{i=1}^{n} 1 / n \mathbf{V}_{i}(\boldsymbol{\beta})$.

\section{Prova:}

Considerando $\frac{1}{\sqrt{n}} \Psi_{n}\left(\boldsymbol{\beta}, \hat{\boldsymbol{\alpha}}_{n}\right)$ como uma função de $\hat{\boldsymbol{\alpha}}_{n}$ e expandindo-a em série de Taylor nas vizinhanças de $\alpha$, segue que

$$
\frac{1}{\sqrt{n}} \boldsymbol{\Psi}_{n}\left(\boldsymbol{\beta}, \hat{\boldsymbol{\alpha}}_{n}\right)=\frac{1}{\sqrt{n}} \boldsymbol{\Psi}_{n}(\boldsymbol{\beta}, \boldsymbol{\alpha})+\frac{1}{n} \frac{\partial \boldsymbol{\Psi}_{n}}{\partial \boldsymbol{\alpha}}(\boldsymbol{\beta}, \boldsymbol{\alpha}) \sqrt{n}\left(\hat{\boldsymbol{\alpha}}_{n}-\boldsymbol{\alpha}\right)+\frac{1}{\sqrt{n}} O_{p}\left(\frac{1}{\sqrt{n}}\right) \text {. }
$$

Note que $\frac{1}{n} \frac{\partial \Psi_{n}}{\partial \boldsymbol{\alpha}}(\boldsymbol{\beta}, \boldsymbol{\alpha})=\frac{1}{n} \sum_{i=1}^{n} \frac{\partial \boldsymbol{\psi}_{i}}{\partial \boldsymbol{\alpha}}(\boldsymbol{\beta}, \boldsymbol{\alpha})$. Como $\frac{\partial \Psi_{n}}{\partial \boldsymbol{\alpha}}(\boldsymbol{\beta}, \boldsymbol{\alpha})$ depende da variável resposta através de uma combinação linear de $\mathbf{u}_{i}, i=1,2, \ldots, n$, os quais são vetores aleatórios independentes com média zero que satisfazem a condição de Markov, a lei fraca dos grandes números de Markov assegura que $\frac{1}{n} \frac{\partial \Psi_{n}}{\partial \boldsymbol{\alpha}}(\boldsymbol{\beta}, \boldsymbol{\alpha})=o_{p}(1)$. Aplicando esse resultado e o Lema 4 em (3.18), vem que

$$
\frac{1}{\sqrt{n}} \Psi_{n}\left(\boldsymbol{\beta}, \hat{\boldsymbol{\alpha}}_{n}\right)=\frac{1}{\sqrt{n}} \boldsymbol{\Psi}_{n}(\boldsymbol{\beta}, \boldsymbol{\alpha})+o_{p}(1)
$$

Como, por hipótese, $\max _{1 \leq i \leq n} \max _{1 \leq i \leq n} E\left|\Psi_{i j}\right|^{2+\delta}<\infty$ para algum $0<\delta<1$, o teorema de Liapunov garante que

$$
\frac{1}{\sqrt{n}} \boldsymbol{\Psi}_{n}(\boldsymbol{\beta}, \boldsymbol{\alpha}) \stackrel{\mathcal{D}}{\rightarrow} \mathcal{N}_{p}\{0 ; \mathrm{V}(\boldsymbol{\beta})\}
$$

Portanto, segue-se o resultado. o

\footnotetext{
${ }^{4}$ ver em Sen e Singer (1993), por exemplo
} 
Lema 6 . Sejam $\left\{\mathbf{T}_{n}\right\}_{n>1}$ uma sequência de estimadores tais que ${ }^{r} \Gamma_{n} \stackrel{\mathcal{P}}{\rightarrow} \theta$ quando $n \rightarrow \infty ;\left\{g_{n}(\theta)\right\}_{n \geq 1}$ uma sequência de funções mensuráveis, duplamente diferenciáveis tal que $g_{n}(\boldsymbol{\theta}) \stackrel{\mathcal{P}}{\rightarrow} g(\boldsymbol{\theta})$, quando $n \rightarrow \infty$ e $\dot{g}_{n}(\boldsymbol{\theta})=$ $\partial g_{n}(\boldsymbol{\theta}) / \partial \boldsymbol{\theta}=O_{p}(1)$. Então $g_{n}\left(\mathbf{T}_{n}\right) \stackrel{\mathcal{P}}{\rightarrow} g(\boldsymbol{\theta})$.

Prova: Expandindo $g_{n}\left(\mathbf{T}_{n}\right)$ em série de Taylor nas vizinhanças de $\boldsymbol{\theta}$, vem que

$$
g_{n}\left(\mathbf{T}_{n}\right)=g_{n}(\boldsymbol{\theta})+\dot{g}_{n}(\boldsymbol{\theta})\left(\mathbf{T}_{n}-\boldsymbol{\theta}\right)+O\left(\left\|\mathbf{T}_{n}-\boldsymbol{\theta}\right\|\right)=g_{n}(\boldsymbol{\theta})+o_{p}(1) .
$$

Como $g_{n}(\boldsymbol{\theta}) \stackrel{\mathcal{P}}{\rightarrow} g(\boldsymbol{\theta})$, segue-se o resultado. 。

Lema 7 . Seja $\dot{\boldsymbol{\Psi}}_{n}\left(\boldsymbol{\beta}, \hat{\boldsymbol{\alpha}}_{n}\right)=\frac{\partial \boldsymbol{\Psi}_{n}}{\partial \boldsymbol{\beta}}\left(\boldsymbol{\beta}, \hat{\boldsymbol{\alpha}}_{n}\right)$. Então, sob $\mathcal{H}$ e as hipóteses do Lema 6 aplicadas às componentes de $\dot{\boldsymbol{\Psi}}_{n}\left(\boldsymbol{\beta}, \hat{\boldsymbol{\alpha}}_{n}\right)=g_{n}\left(\hat{\boldsymbol{\alpha}}_{n}\right)$, vem que

$$
\frac{1}{n} \dot{\Psi}_{n}\left(\boldsymbol{\beta}, \hat{\alpha}_{n}\right) \stackrel{\mathcal{P}}{\rightarrow} \mathbf{S}
$$

conforme $n$ cresce, onde $\mathbf{S}=\lim _{n \rightarrow \infty} \frac{1}{n} \sum_{i=1}^{n} \mathbf{S}_{i}(\boldsymbol{\beta})$.

Prova: Consequência imediata das hipóteses. o

Finalmente, pode-se mostrar o Teorema 6.

\section{Prova do Teorema 6:}

Usando as notações e definições dos Lemas 4,5 e $7, \Psi_{n}\left(\boldsymbol{\beta}, \hat{\boldsymbol{\alpha}}_{n}(\boldsymbol{\beta})\right)$ pode ser expandida ao redor de $\boldsymbol{\beta}_{0}$ (suponha que esse seja o verdadeiro valor do parâmetro), obtendo-se

$$
\boldsymbol{\Psi}_{n}\left(\boldsymbol{\beta}, \hat{\boldsymbol{\alpha}}_{n}\right)=\boldsymbol{\Psi}_{n}\left(\boldsymbol{\beta}_{0}, \hat{\boldsymbol{\alpha}}_{n}\right)+\dot{\boldsymbol{\Psi}}_{n}\left(\boldsymbol{\beta}_{0}, \hat{\boldsymbol{\alpha}}_{n}\right)\left(\boldsymbol{\beta}-\boldsymbol{\beta}_{0}\right)+O\left(\left\|\boldsymbol{\beta}-\boldsymbol{\beta}_{0}\right\|\right)
$$

onde $\dot{\Psi}_{n}=\frac{\partial \Psi_{n}}{\partial \boldsymbol{\beta}^{T}}$. Avaliando-se a função no ponto $\hat{\boldsymbol{\beta}}_{n}$ ao redor de $\boldsymbol{\beta}_{0}$ e utilizando-se a hipótese de limitação em probabilidade de $\hat{\beta}_{n}-\beta_{0}$, vem que

$$
\Psi_{n}\left(\boldsymbol{\beta}, \hat{\alpha}_{n}\right)+\dot{\Psi}_{n}\left(\boldsymbol{\beta}, \hat{\alpha}_{n}\right)\left(\hat{\boldsymbol{\beta}}_{n}-\boldsymbol{\beta}\right)+O_{p}(1)=0
$$

Logo,

$$
\hat{\boldsymbol{\beta}}_{n}-\boldsymbol{\beta}=-\left\{\dot{\boldsymbol{\Psi}}_{n}\left(\boldsymbol{\beta}_{0}\right)\right\}^{-1} \boldsymbol{\Psi}_{n}\left(\boldsymbol{\beta}_{0}\right)+\left\{\dot{\boldsymbol{\Psi}}_{n}\left(\boldsymbol{\beta}_{0}\right)\right\}^{-1} O_{p}(1)
$$


consequentemente,

$$
\sqrt{n}\left(\hat{\boldsymbol{\beta}}_{n}-\boldsymbol{\beta}\right)=-\left\{\frac{1}{n} \dot{\boldsymbol{\Psi}}_{n}\left(\boldsymbol{\beta}_{0}\right)\right\}^{-1}\left\{\frac{1}{\sqrt{n}} \boldsymbol{\Psi}_{n}\left(\boldsymbol{\beta}_{0}\right)\right\}+o_{p}(1) .
$$

Aplicando-se os Lemas 5 e 7, demonstra-se o teorenı. 0

\subsection{Exemplos}

Nesta seção é discutida a aplicação da metodologia desenvolvida a alguns modelos de dispersão.

\subsubsection{Aplicação a modelos exponenciais de dispersão}

Como foi salientado, a aplicação do método desenvolvido aos modelos exponencias de dispersão corresponde à técnica das equações de estimação generalizadas, mais conhecidas na literatura como "GEE", desenvolvida por Liang e Zeger (1986).

Considere as distribuições marginais como pertencentes à família dos modelos exponencias de dispersão com parâmetro canônico $\theta_{i j}$ e parâmetro de dispersão $\lambda$. Partindo-se do desvio unitário para modelos exponenciais de dispersão (1.15) e utilizando-se a notação da Seção 1.2.1, vem que

$$
u_{i j}=-2 v^{-1}\left(\mu_{i j}\right)\left(y_{i j}-\mu_{i j}\right) .
$$

Seja $\mathbf{v}_{i}=\left(v\left(\mu_{i 2}\right), v\left(\mu_{i 2}\right), \ldots, v\left(\mu_{i t}\right)\right)^{\top}$. Considerando-se $\mathbf{W}_{i}=\operatorname{Cov}\left(\mathbf{u}_{i}\right)$, vem que

$$
\begin{aligned}
\mathbf{W}_{i} & =4 \Delta^{-1}\left(\mathbf{v}_{i}\right) \operatorname{Cov}\left(\mathbf{y}_{i}-\boldsymbol{\mu}_{i}\right) \Delta^{-1}\left(\mathbf{v}_{i}\right) \\
& =4 \sigma^{2} \Delta^{-1 / 2}\left(\mathbf{v}_{i}\right) \mathbf{R}_{i} \Delta^{-1 / 2}\left(\mathbf{v}_{i}\right),
\end{aligned}
$$

onde $\mathbf{R}_{i}$ é a matriz de correlação associada a $\mathbf{y}_{i}$. Eventualmente, pode-se supor $\mathbf{R}_{i}=\mathbf{R}, i=1,2, \ldots, n$ e nesse caso, o problema de estimação de $\mathbf{W}_{i}$, reduz-se a estimar $R=\mathbf{R}(\boldsymbol{\alpha})$. 
Assim fazendo, uma função de estimação ótima em $\mathcal{L}$ gerada por $\mathbf{u}_{i}$ é

$$
\begin{aligned}
\boldsymbol{\Psi}_{n}(\boldsymbol{\beta}) & =\sum_{i=1}^{n} \mathbf{X}_{i}^{\top} \mathbf{H}_{i} \Delta^{-1}\left(\mathbf{v}_{i}\right) \mathbf{W}_{i}^{-1} \Delta^{-1}\left(\mathbf{v}_{i}\right)\left(\mathbf{y}_{i}-\boldsymbol{\mu}_{i}\right) \\
& =\sum_{i=1}^{n} \mathbf{X}_{i}^{\top} \mathbf{H}_{i}\left\{\Delta^{-1 / 2}\left(\mathbf{v}_{i}\right) \mathbf{R}_{i}^{-1} \Delta^{-1 / 2}\left(\mathbf{v}_{i}\right)\right\}\left(\mathbf{y}_{i}-\boldsymbol{\mu}_{i}\right) \\
& =\sum_{i=1}^{n} \mathbf{X}_{i}^{\top} \mathbf{H}_{i} \operatorname{Cov}^{-1}\left(\mathbf{y}_{i}\right)\left(\mathbf{y}_{i}-\boldsymbol{\mu}_{i}\right)
\end{aligned}
$$

No caso em que Cov $\left(\mathrm{y}_{i}\right)$ é desconhecida,

$$
\boldsymbol{\Psi}_{n}=\sum_{i=1}^{n} \mathbf{X}_{i}^{\top} \mathbf{H}_{i} \mathbf{W}_{i}^{-1}\left(\hat{\boldsymbol{\alpha}}_{n}\right)\left(\mathrm{y}_{i}\right)\left(\mathrm{y}_{i}-\boldsymbol{\mu}_{i}\right)
$$

como na Seção 3.1.2.

No desenvolvimerito da teoria das "GEE", Liang e Zeger (1986) optaram por modelar o parâmetro canônico como

$$
\theta_{i j}=e\left(\eta_{i j}\right)
$$

onde $e(\cdot)$ é uma função duplamente diferenciável e inversível; já nas equações propostas nesta tese, a modelagem é feita sobre a média da distribuição

$$
\mu_{i j}=g^{-1}\left(\eta_{i j}\right)=h\left(\eta_{i j}\right)
$$

Utilizando resultados apresentados na Seção 1.2.1, vem que $\mu_{i j}=\tau\left(\theta_{i j}\right)$ e, portanto,

$$
\frac{\partial h}{\partial \eta_{i j}}\left(\eta_{i j}\right)=\frac{\partial \tau}{\partial \eta_{i j}}\left(\theta_{i j}\right)=\frac{\partial \tau}{\partial \theta_{i j}}\left(\theta_{i j}\right) \frac{\partial \theta_{i j}}{\partial \eta_{i j}}\left(\eta_{i j}\right)=v\left(\mu_{i j}\right) \dot{e}_{i j}
$$

onde $\dot{e}_{i j}=\frac{\partial \theta_{i j}}{\partial \eta_{i j}}\left(\eta_{i j}\right)$. Aplicando esse resultado a (3.22), segue que

$$
\boldsymbol{\Psi}_{n}(\boldsymbol{\beta})=\sum_{i=1}^{n} \mathbf{X}_{i}^{\top} \mathbf{E}_{i} \Delta\left(\mathbf{v}_{i}\right) \mathbf{W}_{i}^{-1}\left(\hat{\alpha}_{n}\right)\left(\mathrm{y}_{i}-\boldsymbol{\mu}_{i}\right)
$$

onde $\mathbf{E}_{i}=\operatorname{diag}\left\{\dot{e}_{i 1}, \dot{e}_{i 2}, \ldots, \dot{e}_{i t}\right\}$, que é a expressão usualmente apresentada como "GEE", na literatura. 
Liang e Zeger (1986) sugerem que $\lambda$ e $\alpha$ sejam estimados utilizando-se propriedades dos resíduos de Pearson. Seja

$$
\hat{r}_{i j}=\frac{y_{i j}-\dot{a}\left(\hat{\theta}_{i j}\right)}{\left\{\ddot{a}\left(\ddot{\theta}_{i j}\right)\right\}^{1 / 2}},
$$

onde $\hat{r}_{i j}$ é o resíduo de Pearson da observação $y_{i j}$. Se o quarto momento de $y_{i j}$ é finito, demonstra-se que

$$
\hat{\lambda}=\left\{\sum_{i=1}^{n} \sum_{j=1}^{t} \hat{r}_{i j}^{2} /(n t-p)\right\}^{-1}
$$

é um estimador $\sqrt{n}$-consistente de $\lambda$; trata-se, na verdade, do estimador de Pearson apresentado na Seção 3.1.3.

Um estimador $\sqrt{n}$-consistente da componente $(j, k)$ de $\mathbf{R}$ pode ser obtido por $\sum_{i=1}^{n} \hat{r}_{i j} \hat{r}_{i k} /(n-p)$. Liang e Zeger (1986) apresentam estimadores de $\boldsymbol{\alpha}$ quando estruturas de correlação especiais são consideradas.

Há na literatura estatística vários estudos de simulação envolvendo essa metodologia e descreveremos alguns deles.

Drum e McCullagh, em Fitzmaurice et al. (1993), afirmam que o estimador sanduíche da matriz de covariância assintótica do estimador de $\boldsymbol{\beta}$, embora consistente, perde a eficiência quando o tamanho amostral é pequeno ou moderado. Firth, em Liang et al. (1992), diz que o estimador sanduíche está sujeito a vícios, principalmente em planejamentos não balanceados.

Liang et al. (1992) e Fitzmaurice et al. (1993) alertam que, embora consistentes, os estimadores de $\beta$ e o sanduíche tendem a ser ineficientes ao se assumir uma estrutura de correlação muito diferente da real. Albert e McShane (1995) concluem que a especificação correta dessa estrutura pode diminuir o vício das estimativas.

Liang e Zeger (1986) comparam a eficiência assintótica dos estimadores obtidos através das funções de estimação (3.2) e (3.7) em dois modelos simples e concluem que quanto mais forte a correlação entre as observações, mais eficiente é o estimador obtido a partir de (3.7); uma conclusão análoga pode ser encontrada em Albert e McShane (1995).

Pepe e Anderson (1994) ilustram, através de um estudo de simulação no qual $\mathbf{R}$ é considerada conhecida que se $E\left(y_{i j} \mid \mathbf{x}_{i j}\right) \neq E\left(y_{i j} \mid \mathbf{x}_{i k}, k \leq j\right)$, 
$i=1,2, \ldots, n$ e $j=1,2, \ldots, t$, as estimativas de $\boldsymbol{\beta}$ podem ser seriamente viesadas.

Liang et al. (1992) e Fitzmaurice et al. (1993) comparam (3.14) e (3.13). Eles concluem que a grande vantagem de (3.13) é que as estimativas de $\boldsymbol{\beta}$ e $\alpha$ são mais eficisntes do que as obtidas por (3.14), uma vez que a inodelagem da média e da estrutura de correlação estejam corretas. Uma fraqueza de (3.13) e de (3.14) é que as estimativas de $\boldsymbol{\beta}$ podem ser não consistentes se a estrutura de correlação for mal especificada; nessa situação o uso de (3.7) é preferível, já que as correspondentes estimativas de $\boldsymbol{\beta}$ são consistentes mesmo nessa situação adversa.

Firth, comentando Liang et al. (1992), sugere um algoritmo híbrido para a estimação dos parâmetros:

a. estime $\boldsymbol{\beta}$ utilizando (3.14);

b. assuma a estimativa obtida em (a.) e substitua-a em (3.13), obtendo uma estimativa de $\boldsymbol{\alpha}$;

c. repita (a.) e (b.) até que se consiga a convergência.

Lipsitz et al. (1994) comparam, em um estudo de simulação envolvendo dados binários, a performance dos estimadores obtidos através de (3.13) com estimadores obtidos executando-se apenas o primeiro passo do processo iterativo que leva à sua obtenção. Eles concluem que, quando (3.13) converge, o desempenho dos métodos é equivalente. Eles também notaram que o processo iterativo não converge com uma certa frequência, em situações envolvendo altas correlações e pequenos tamanhos amostrais e, nesses casos, a performance do método alternativo mostrou-se satisfatória.

Aplicações dessa técnica podem ser encontradas em Zeger e Liang (1986), Zeger, Liang e Self (1985), Zeger, Liang e Albert (1988), Prentice (1988), Prentice e Zhao (1991), Carr e Chi (1992), Liang et al. (1992), Whittemore e Gong (1994), Cologne et al. (1993), Lee et al (1993), Miller et al (1993), Kenward et al (1994), Liang e Hanfelt (1994) e Albert e McShane (1995). Revisões sobre o assunto podem ser encontradas em Liang et al. (1992), Fitzmaurice et al. (1993) e Diggle et al. (1994).

Extensões dessa metodologia para a estimação de modelos de séries temporais podem ser encontradas em Zeger e Qaqish (1988) e Li (1995). 


\subsubsection{Aplicação a dados circulares}

Por simplicidade, optou-se por trabalhar com a forma (1.3) da distribuição von Mises e pequenas adaptações são necessárias para adaptar as fórmulas já apresentadas.

Considere $y_{i j} \sim \mathcal{M}\left(\mu_{i j} ; w_{i j} \lambda\right)$ e, sem perda de generalidade, assuma que $y_{i j}$ e $\mu_{i j}$ variem no intervalo $(-\pi, \pi]$.

\section{Modelagem da média circular}

A partir da densidade da distribuição von Mises, conclui-se que $\frac{\partial d}{\partial \mu_{i j}}\left(y_{i j} ; \mu_{i j}\right)$ é proporcional a sen $\left(y_{i j}-\mu_{i j}\right)$. Por simplicidade, sem prejuizo dos resultados subsequentes, assuma $u_{i j}=\operatorname{sen}\left(y_{i j}-\mu_{i j}\right)$. Como no estudo de variáveis angulares há grande uso de funções trigonométricas, $u_{i j}$ têm uma forma bastante conveniente. As componentes das matrizes $\mathbf{N}_{i}, i=1,2, \ldots, n$, são facilmente obtidas, uma vez que $E\left(u_{i j}\right)=-E\left\{\cos \left(y_{i j}-\mu_{i j}\right)\right\}=-A_{1}\left(w_{i j} \lambda\right)=$ $-\frac{I_{1}\left(w_{i j} \lambda\right)}{I_{0}\left(w_{i j} \lambda\right)}$. Definindo $\mathbf{e}_{i}$ como um vetor $t$-dimensional com componentes $\mathcal{A}_{1}\left(w_{i j} \lambda\right)$, se os pesos $w_{i j}=w, i=1,2, \ldots, n, j=1,2, \ldots, t$, as matrizes $\mathbf{N}_{i}$ podem ser retiradas da função de estimação sem prejuízo das estimativas. Nesse caso,

$$
\Psi_{n}(\boldsymbol{\beta})=\sum_{i=1}^{n} \mathbf{X}_{i} \mathbf{H}_{i} \mathbf{W}_{i}^{-1} \mathbf{u}_{i}
$$

\section{Adaptação para o modelo (1.5)}

O modelo de regressão (1.5) constitui um dos mais recentes avanços na área de modelos de regressão para dados circulares. Os resultados até então desenvolvidos devem sofrer algum tipo de modificação para poderem acomodar essa modelagem. Considere pois

$$
\mu_{i j}=\beta_{0}+g\left(\eta_{i j}\right), \quad \eta_{i j}=\mathbf{x}_{i j}^{\top} \beta
$$

$\operatorname{com} \theta=\left(\boldsymbol{\beta}^{\top}, \beta_{0}\right)^{\top}$ e $\mathbf{u}_{i}, i=1, \ldots, n$, como a função geradora da classe $\mathcal{L}$. Utilizando-se (2.1), vem que a função de estimação ótima nessa classe é dada por

$$
\Lambda_{n}(\theta)=\sum_{i=1}^{n}\left(\begin{array}{c}
\mathbf{D}_{i}^{* \top} \\
\delta^{\top}\left(\mathbf{N}_{i}\right)
\end{array}\right) \mathbf{W}_{i} \mathbf{u}_{i}
$$


Definindo a componente $(j, k)$ da matriz $\mathbf{W}_{i}$ por $w_{i j k}$ e igualando-se (3.23) a zero, vem que

$$
\delta^{\top}\left(\mathbf{N}_{i}\right) \mathbf{W}_{i} \mathbf{u}_{i}=\sum_{i=1}^{n} \sum_{i=1}^{t} \sum_{k=1}^{t} \mathcal{A}_{1}\left(\omega_{j k i} \lambda\right) w_{i j k} \operatorname{sen}\left\{y_{i k}-\hat{\beta}_{0}-g\left(\hat{\eta}_{i k}\right)\right\}=0 .
$$

Utilizando-se propriedades trigonométricas, vem que a equação anterior equivale a

$$
\begin{aligned}
\sum_{i=1}^{n} \sum_{j=1}^{t} \sum_{k=1}^{t} \mathcal{A}_{1}\left(\omega_{j k i} \lambda\right) w_{i j k} \operatorname{sen}\left\{y_{i k}-g\left(\hat{\eta}_{i k}\right)\right\} \\
=\tan \left(\hat{\beta}_{0}\right) \sum_{i=1}^{n} \sum_{j=1}^{t} \sum_{k=1}^{t} \mathcal{A}_{1}\left(\omega_{j k i} \lambda\right) w_{i j k} \operatorname{sen}\left\{y_{i k}-g\left(\hat{\eta}_{i k}\right)\right\} .
\end{aligned}
$$

Definindo-se

$$
\begin{aligned}
& S=\sum_{i=1}^{n} \sum_{j=1}^{t} \sum_{k=1}^{t} \mathcal{A}_{1}\left(\omega_{j k i} \lambda\right) w_{i j k} \operatorname{sen}\left\{y_{i k}-g\left(\hat{\eta}_{i k}\right)\right\} \\
& C=\sum_{i=1}^{n} \sum_{j=1}^{t} \sum_{k=1}^{t} \mathcal{A}_{1}\left(\omega_{j k i} \lambda\right) w_{i j k} \cos \left\{y_{i k}-g\left(\hat{\eta}_{i k}\right)\right\}
\end{aligned}
$$

tem-se, então, que

$$
\hat{\beta}_{0}=\left\{\begin{array}{l}
\arctan \left(\frac{S}{C}\right), C \geq 0 \\
\arctan \left(\frac{S}{C}\right)+\pi, C<0, S \geq 0, \\
\arctan \left(\frac{S}{C}\right)-\pi, C<0, S<0 .
\end{array}\right.
$$

Sob as condições do Teorema $6, \hat{\theta}_{n}$, raiz de $\Lambda_{n}(\theta)$, é consistente e

$$
\sqrt{n}\left(\hat{\boldsymbol{\theta}}_{n}-\theta\right) \stackrel{\mathcal{D}}{\rightarrow} \mathcal{N}_{p+1}\left(0 ; \overline{\mathbf{J}}^{-1}\right)
$$

quando $n \rightarrow \infty$, onde $\overline{\mathbf{J}}=\mathbf{S V}^{-1} \mathbf{S}^{\top}$ com

$$
\mathbf{S}=\lim _{n \rightarrow \infty} \frac{1}{n} \sum_{i=1}^{n}\left[\begin{array}{cc}
\mathbf{D}_{i}^{* \top} \mathbf{W}_{i}^{-1} \mathbf{D}_{i}^{*} & \mathbf{D}_{i}^{* \top} \mathbf{W}_{i} \delta\left(\mathbf{N}_{i}\right) \\
\delta^{\top}\left(\mathbf{N}_{i}\right) \mathbf{W}_{i} \mathbf{D}_{i}^{* \top} & \delta^{\top}\left(\mathbf{N}_{i}\right) \mathbf{W}_{i} \delta\left(\mathbf{N}_{i}\right)
\end{array}\right]
$$


$\mathrm{e}$

$$
\mathbf{V}=\lim _{n \rightarrow \infty} \frac{1}{n} \sum_{i=1}^{n}\left(\begin{array}{ll}
\mathbf{V}_{11 i} & \mathbf{V}_{12 i} \\
\mathbf{V}_{12 i}^{\top} & V_{22 i}
\end{array}\right)
$$

onde

$$
\begin{gathered}
\mathbf{V}_{11 i}=\mathbf{D}_{i}^{* \top} \mathbf{W}_{i}^{-1} \operatorname{Cov}\left(\mathbf{u}_{i}\right) \mathbf{W}_{i}^{-1} \mathbf{D}_{i}^{*} \\
\mathbf{V}_{12 i}=\mathbf{D}_{i}^{* \top} \mathbf{W}_{i}^{-1} \operatorname{Cov}\left(\mathbf{u}_{i}\right) \mathbf{W}_{i}^{-1} \delta\left(\mathbf{N}_{i}\right)
\end{gathered}
$$

$\mathrm{e}$

$$
V_{22 i}=\delta^{\top}\left(\mathbf{N}_{i}\right) \mathbf{W}_{i}^{-1} \operatorname{Cov}\left(\mathbf{u}_{i}\right) \mathbf{W}_{i}^{-1} \delta\left(\mathbf{N}_{i}\right)
$$

\subsection{Modelo misto}

Nesta seção são propostas estratégias de análise para a estimação de modelos de regressão mistos para dados longitudinais. A importância de tais modelos para a análise de dados circulares foi discutida na. Seção 1.1.4.

Seja $y_{i j} \sim \mathcal{M D}\left(\mu_{i j} ; w_{i j}^{-1} \sigma^{2}\right)$, onde $\mu_{i j}, \sigma^{2}$ e $w_{i j}, i=1,2, \ldots n$ e $j=$ $1,2, \ldots t$ são parâmetros desconhecidos. Suponha a modelagem do parâmetro de posição descrita na Seção 3.1 e a existência de uma função, duplamente diferenciável e inversível $q$, tal que

$$
q\left(w_{i j}\right)=\xi_{i j}=\sum_{l=1}^{m} z_{i j l} \gamma_{l}
$$

onde $z_{i j l}$ são covariáveis fixas, $\gamma_{l}$ são parâmetros desconhecidos e a imagem de $q(\cdot)$ é um subconjunto de $\Re_{+}, i=1,2, \ldots, n, j=1,2, \ldots, t$ e $l=1,2, \ldots, m$. Defina $\boldsymbol{\gamma}=\left(\gamma_{1}, \ldots, \gamma_{r}\right)^{\top}, \mathbf{z}_{i j}=\left(z_{i j 1}, \ldots, z_{i j m}\right)^{\top}$ e $\mathbf{Z}_{i}=\left(\mathbf{z}_{i 1}, \ldots, \mathbf{z}_{i t}\right)^{\top}$.

No caso de independência entre as observações de um mesmo indivíduo, a função escore de $\theta=\left(\boldsymbol{\beta}^{\top}, \gamma^{\top}, \sigma^{2}\right)^{\top}$ é dada por

$$
\boldsymbol{\Upsilon}_{n}^{I}(\boldsymbol{\theta})=\frac{\partial L}{\partial \boldsymbol{\theta}}(\boldsymbol{\theta})=\sum_{i=1}^{n} \boldsymbol{v}_{i}^{I}(\boldsymbol{\theta})=\sum_{i=1}^{n}\left(\begin{array}{c}
\psi_{i}^{I} \\
\mathrm{G}_{i} \\
s_{i}
\end{array}\right)
$$


onde $\psi_{i}^{I}$ é como em (3.2),

$$
\begin{aligned}
& \mathbf{G}_{i}=-\mathbf{Z}_{i}^{\top} \mathbf{F}_{i} \varepsilon_{i}, \\
& \varepsilon_{i}=\left(\sigma^{2} \mathbf{p}_{1 i}+\frac{1}{2 \sigma^{2}} \mathbf{d}_{i}\right), \\
& \mathbf{p}_{1 i}=\mathbf{A}_{i}^{-2} \Delta^{-i}\left(\mathbf{a}_{i}\right) \dot{\mathbf{a}}_{i}, \\
& \mathbf{F}_{i}=\left(\dot{f}_{i 1}, \ldots, \dot{f}_{i t}\right)^{\top}, \dot{f}_{i j}=\left.\frac{\partial w_{i j}}{\partial x}(x)\right|_{x=\xi_{i j}}, \\
& s_{i}=\sum_{j=1}^{t}\left\{\frac{\dot{a}_{i j}}{a_{i j} w_{i j}}+\frac{w_{i j}}{2 \sigma^{4}} d_{i j}\right\}=\dot{\mathbf{a}}_{i}^{\top} \mathbf{A}_{i}^{-1} \delta\left\{\Delta^{-1}\left(\mathbf{a}_{i}\right)\right\}+\frac{1}{2 \sigma^{4}} \delta^{\top}\left(\mathbf{A}_{i}\right) \mathbf{d}_{i},
\end{aligned}
$$

$\mathbf{a}_{i}, \dot{\mathbf{a}}_{i}, \ddot{\mathbf{a}}_{i}, \mathrm{~d}_{i}$ são vetores $t$-dimensionais com componentes, respectivamente, $a_{i j}=a\left(y_{i j}, \sigma^{2} / w_{i j}\right), \dot{a}_{i j}=\left.\frac{\partial a}{\partial x}\left(y_{i j}, x\right)\right|_{x=w_{i j}^{-1} \sigma^{2}}, \ddot{a}_{i j}=\left.\frac{\partial^{2} a}{\partial x^{2}}\left(y_{i j}, x\right)\right|_{x=w_{i j}^{-1} \sigma^{2}} \mathrm{e}$ $d_{i j}=d\left(y_{i j}, \mu_{i j}\right), j=1,2 \ldots, t$.

Seja $\hat{\theta}_{n}^{I}$ a raiz de $\Upsilon_{n}^{I}$. Pode-se demonstrar, sob condições gerais de regularidade, que $\hat{\boldsymbol{\theta}}_{n}^{I}$ é consistente e assintoticamente normal com matriz de covariância assintótica dada pelo limite do inverso da matriz de informação de Godambe de $\Upsilon_{n}^{I}$, quando $n$ cresce para o infinito ${ }^{5}$.

A função de estimação ótima na classe $\mathcal{L}$, gerada por $\left(\mathbf{u}_{i}^{\top}, \varepsilon_{i}^{\top}, s_{i}\right)^{\top}$, é dada por

com

$$
\boldsymbol{\Upsilon}_{n}^{*}(\boldsymbol{\theta})=\sum_{i=1}^{n} E\left(\begin{array}{lll}
\frac{\partial \mathbf{u}_{i}}{\partial \boldsymbol{\beta}^{\top}} & \frac{\partial \mathbf{u}_{i}}{\partial \boldsymbol{\gamma}^{\top}} & \frac{\partial \mathbf{u}_{i}}{\partial \sigma^{2}} \\
\frac{\partial \varepsilon_{i}}{\partial \boldsymbol{\beta}^{\top}} & \frac{\partial \varepsilon_{i}}{\partial \boldsymbol{\gamma}^{\top}} & \frac{\partial \varepsilon_{i}}{\partial \sigma^{2}} \\
\frac{\partial s_{i}}{\partial \boldsymbol{\beta}^{\top}} & \frac{\partial s_{i}}{\partial \boldsymbol{\gamma}^{\top}} & \frac{\partial s_{i}}{\partial \sigma^{2}}
\end{array}\right) \mathbf{W}_{i}^{*-1}\left(\begin{array}{c}
\mathbf{u}_{i} \\
\varepsilon_{i} \\
s_{i}
\end{array}\right)
$$

$$
\mathbf{W}_{i}^{*}=\operatorname{Cov}\left(\begin{array}{c}
\mathbf{u}_{i} \\
\varepsilon_{i} \\
s_{i}
\end{array}\right)=E\left(\begin{array}{ccc}
\mathbf{u}_{i} \mathbf{u}_{i}^{\top} & \mathbf{u}_{i} \varepsilon_{i}^{\top} & \mathbf{u}_{i} s_{i} \\
\varepsilon_{i} \mathbf{u}_{i}^{\top} & \varepsilon_{i} \varepsilon_{i}^{\top} & \varepsilon_{i} s_{i} \\
s_{i} \mathbf{u}_{i}^{\top} & s_{i} \varepsilon_{i}^{\top} & s_{i}^{2}
\end{array}\right) .
$$

No caso em que $\mathbf{W}_{i}^{*}$ é desconhecida, pode-se utilizar um procedimento similar ao proposto na Seção 3.1.2, considerando suas componentes como parâmetros de perturbação e estimá-las consistentemente. Desse modo, satisfeitas as condições do Teoremá 6 , as raízes da funçào de estimação formam uma sequência consistente e assintoticamente normal de estimadores. A desvantagem dessa abordagem é o grande número de parâmetros de perturbação

\footnotetext{
${ }^{5}$ ver Teorema 3 ou 4 , por exemplo.
} 
introduzidos no modelo e a eventual necessidade de novas suposições sobre a estrutura de dependência dos dados. Simplificações podem ser sugeridas no sentido de minimizar esse problema, se for abandonada a propriedade de otimalidade da função de estimação. Assim, pode-se sugerir as seguintes funções de estimação alternativas:

$$
\begin{aligned}
\mathbf{\Upsilon}_{n}^{o}(\boldsymbol{\theta}) & =\sum_{i=1}^{n} E\left(\begin{array}{ccc}
\frac{\partial \mathbf{u}_{i}}{\partial \boldsymbol{\beta}^{\top}} & 0 & 0 \\
0 & \frac{\partial \varepsilon_{i}}{\partial \boldsymbol{\gamma}^{\top}} & 0 \\
0 & 0 & \frac{\partial s_{i}}{\partial \sigma^{2}}
\end{array}\right) \mathbf{W}_{i}^{o-1}\left(\begin{array}{c}
\mathbf{u}_{i} \\
\varepsilon_{i} \\
s_{i}
\end{array}\right) \\
& =\sum_{i=1}^{n}\left[\begin{array}{c}
\lambda \mathbf{Z}_{i}^{\top} \mathbf{F}_{i} \mathbf{M}_{i} \operatorname{Cov}^{-1}\left(\varepsilon_{i}\right) \varepsilon_{i} \\
\sum_{j=1}^{t} \frac{w_{i j}\left\{\dot{\mathbf{a}}_{i}^{\top} \mathbf{A}_{i}^{-1} \delta\left\{\Delta^{-1}\left(\mathbf{a}_{i}\right)\right\}+\frac{1}{2 \sigma^{4}} \delta^{\top}\left(\mathbf{A}_{i}\right) \mathbf{c}_{i}^{\mathbf{\alpha}}\right\}}{\mathbf{1}_{t}^{\top} \operatorname{Cov}\left(\varepsilon_{i}\right) \mathbf{1}}
\end{array}\right],
\end{aligned}
$$

com $\mathbf{M}_{i}=E\left\{\Delta\left(\dot{\varepsilon}_{i}\right)\right\}$, onde $\dot{\varepsilon}_{i}$ é um vetor $t \times 1$ cuja $j$-ésima componente é a derivada da $j$-ésima componente de $\varepsilon_{i}$ em relação a $\xi_{i j}$

$$
\mathbf{W}_{i}^{o}=E\left(\begin{array}{ccc}
\mathbf{u}_{i} \mathbf{u}_{i}^{\top} & 0 & 0 \\
0 & \varepsilon_{i} \varepsilon_{i}^{\top} & 0 \\
0 & 0 & s_{i}^{2}
\end{array}\right)
$$

e $\mathbf{1}_{t}$ é um vetor coluna de dimensão $t$ com todas as componentes iguais a um, ou mesmo

$$
\Upsilon_{n}(\theta)=\sum_{i=1}^{n}\left[\begin{array}{c}
\mathbf{X}_{i}^{\top} \mathbf{H}_{i} \mathbf{N}_{i} \mathbf{W}_{i}^{-1} \mathbf{u}_{i} \\
\mathbf{Z}_{i}^{\top} \mathbf{F}_{i} \varepsilon_{i} \\
\dot{\mathbf{a}}_{i}^{\top} \mathbf{A}_{i}^{-1} \delta\left\{\Delta^{-1}\left(\mathbf{a}_{i}\right)\right\}+\frac{1}{2 \sigma^{4}} \delta^{\top}\left(\mathbf{A}_{i}\right) \mathbf{d}_{i}
\end{array}\right]
$$

Espera-se que quanto mais próxima for a função de estimação da ótima em $\mathcal{L}$, melhores serão seus resultados em termos da eficiência de seus estimadores.

O Teorema 3 (ou o 4) fornece, sob condições gerais, a forma da matriz de covariância assintótica dos estimadores de $\theta$ obtidos a partir das funções de estimação apresentadas quando as matrizes de covariância envolvidas nas funções de estimação são conhecidas. Essas matrizes podem ser consistentemente estimadas pelos correspondentes estimadores "sanduíche". Caso as 
matrizes de covariância envolvidas nas funções de estimação sejam desconhecidas, pode-se proceder como na Seção 3.1.2.

Apenas como ilustração, serão apresentadas as componentes da matriz de covariância assintótica dos estimadores e detalhes das alterações necessárias quando a matriz de covariância, $\mathbf{W}^{-1}$, é desconhecida para a função (3.26). Esses resultados podem ser diretamente generalizados para as outras funções apresentadas sem majores complicações.

A matriz de informação de Godambe associada à função de estimação $\boldsymbol{v}_{i}(\theta)$, é dada por

$$
J_{v_{i}}(\theta)=\mathbf{S}_{i}(\theta) \mathbf{V}_{i}^{-1}(\theta) \mathbf{S}_{i}^{\top}(\theta)
$$

onde

$$
\mathbf{S}_{i}(\theta)=\left(\begin{array}{ccc}
\mathbf{S}_{11 i} & \mathbf{0} & \mathbf{0} \\
\mathbf{0} & \mathbf{S}_{22 i} & \mathbf{S}_{23 i} \\
\mathbf{0} & \mathbf{S}_{32 i} & \mathbf{S}_{33 i}
\end{array}\right) \text { e } \mathbf{V}_{\mathbf{i}}(\theta)=\left(\begin{array}{lll}
\mathbf{V}_{11 i} & \mathbf{V}_{12 i} & \mathbf{V}_{13 i} \\
\mathbf{V}_{21 i} & \mathbf{V}_{22 i} & \mathbf{V}_{23 i} \\
\mathbf{V}_{31 i} & \mathbf{V}_{32 i} & \mathbf{V}_{33 i}
\end{array}\right)
$$

As expressões das componentes dessas matrizes são dadas no Apêndice C.

Essas expressões tornam-se consideravelmente mais simples se a variável resposta for um modelo próprio de dispersão. Nesse caso, $a_{i j}$ pode ser fatorada em uma parte que depende só dos dados (que pode ser excluída das funções de estimação) e outra só dos parâmetro (que é mantida nas funções).

Corolário 3 . Sob as condições do Teorema 3, considerando-se $\hat{\theta}_{n}$ a solução de $\boldsymbol{\Upsilon}_{n}(\boldsymbol{\theta})=0$, vem que

$$
\sqrt{n}\left(\hat{\theta}_{n}-\boldsymbol{\theta}\right) \stackrel{\mathcal{D}}{\rightarrow} \mathcal{N}\left\{0, n \mathbf{S}^{-1}(\boldsymbol{\theta}) \mathbf{V}(\boldsymbol{\theta}) \mathbf{S}^{-\top}(\boldsymbol{\theta})\right\}
$$

onde

$$
\mathbf{S}(\theta)=\sum_{i=1}^{n} \mathbf{S}_{i}(\theta) \text { e } \mathbf{V}(\theta)=\sum_{\mathrm{i}=1}^{\mathrm{n}} \mathrm{V}_{\mathrm{i}}(\boldsymbol{\theta})
$$

Quando $W_{i}$ for desconhecida, pode-se utilizar soluçôes análogas às utilizadas na Seção 3.1. Basicamente, pode-se considerar a existência de um vetor paramétrico $\alpha$ que define $\mathbf{W}_{i}$, ou seja, $\mathbf{W}_{i}=\mathbf{W}_{i}(\boldsymbol{\alpha})$. Dado $\theta$, pode-se considerar um estimador $\hat{\alpha}$ de $\boldsymbol{\alpha}$, tal que $\hat{\boldsymbol{\alpha}}_{n}=\hat{\boldsymbol{\alpha}}(\theta)$ seja $\sqrt{n}$-consistente. $\mathrm{O}$ próximo passo é redefinir o novo conjunto de equações de estimação como

$$
\Upsilon\{\theta, \hat{\alpha}(\theta)\}=0 .
$$

O próximo teorema assegura, sob condições gerais de regularidade, que a solução de (3.28) é assintoticamente normal. 
Teorema 7 . Seja $\hat{\theta}_{n}$ a solução de (3.28). Sob condições gerais de regularidade, sendo $\left\|\hat{\theta}_{n}-\theta\right\|=O_{p}(1)$ e assumindo que $\hat{\alpha}=\hat{\alpha}(\theta)$ é um estimador $\sqrt{n}$-consistente de $\alpha$ dado $\theta$, tem-se que

$$
\sqrt{n}\left(\hat{\theta}_{n}-\theta\right) \stackrel{\mathcal{D}}{\rightarrow} \mathcal{N}\left(0, \overline{\mathbf{J}}^{-1}\right),
$$

quando $n \rightarrow \infty$, onde

$$
\overline{\mathbf{J}}^{-1}=\lim _{n \rightarrow \infty} n\left\{\sum_{i=1}^{n} \mathbf{S}_{i}(\boldsymbol{\theta})\right\}^{-1}\left\{\sum_{i=1}^{n} \mathbf{V}_{i}(\boldsymbol{\theta})\right\}\left\{\sum_{i=1}^{n} \mathbf{S}_{i}(\boldsymbol{\theta})\right\}^{-\mathrm{T}},
$$

$\mathbf{S}_{i}$ e $\mathbf{V}_{i}$ como em (3.27).

Prova: Similar à do Teorema 6 e à do resultado análogo encontrado em Prentice e Zhao (1991).

Como na Seção 3.1, a abordagem na qual adiciona-se novas equações de estimação para a estimação de $\alpha$ pode ser utilizada.

\subsubsection{Algoritmos de estimação}

Denotando o valor da estimativa $\theta$ no passo $l$ do processo iterativo por $\theta^{(l)}$, tem-se que o método de Newton modificado é dado por

$$
\theta^{(l+1)}=\theta^{(l)}-\mathbf{S}^{-1}\left(\theta^{(l)}\right) \Psi\left(\theta^{(l)}\right) .
$$

$\mathrm{O}$ algoritmo de Newton é obtido substituindo-se $\mathbf{S}$ pela derivada parcial da função de estimação em relação a $\boldsymbol{\theta}^{\top}$.

Ao final de cada passo, deve-se obter uma estimativa atualizada de $\mathbf{W}_{i}$.

Erros-padrão de estimação e testes de hipóteses para a hipótese linear geral podem ser obtidos como na Seção 3.1.

Vale ressaltar que, apesar das funções propostas terem sido desenvolvidas tendo por base os modelos de dispersão, elas podem ser utilizadas em situações mais gerais, onde se desconheça a distribuição marginal e são feitas suposições sobre $E\left(\mathbf{u}_{i}\right)$, por exemplo. Como ilustração. retome o caso de variáveis circulares; prova-se que para qualquer variável circular contínua $E\left\{\operatorname{sen}\left(y_{i j}-\mu_{i j}\right)\right\}=0$, assim, em princípio ela pode ter sua média circular estimada por uma das funções de estimação aqui desenvolvidas. 


\subsubsection{Aplicação a dados circulares}

No caso da modelagem mista, para a função de estimação (3.26),tem-se

$$
\begin{aligned}
& \boldsymbol{\Psi}_{n}(\boldsymbol{\beta})=\sum_{i=1}^{n} \mathbf{X}_{i}^{\top} \mathbf{H}_{i} \mathbf{N}_{i} \mathbf{W}_{i}^{-1} \mathbf{u}_{i}, \\
& \mathbf{G}_{i}=\lambda \sum_{i=1}^{n} \mathbf{Z}_{i}^{\top} \mathbf{F}_{i}\left\{\varepsilon_{i}\right\}, \\
& s_{i}=\sum_{i=1}^{n} \sum_{j=1}^{t} w_{i j}\left\{\cos \left(y_{i j}-\mu_{i j}\right)-\mathcal{A}_{1}\left(w_{i j} \lambda\right)\right\},
\end{aligned}
$$

onde $\varepsilon_{i}=\mathbf{d}_{i}-\delta\left(\mathbf{N}_{i}\right), \mathbf{d}_{i}$ é um vetor $t$-dimensional que tem $\cos \left(y_{i j}-\mu_{i j}\right)$ por componentes e as outras expressões estão definidas na Seção 3.2.2.

A partir dessa função de estimação pode-se, sem maiores dificuldades, obter a função correspondente a (3.25), bastando, para isso, substituir $\mathrm{G}_{i}$ e $s_{i}$ por

$$
\begin{aligned}
\mathbf{G}_{i}^{*} & =\lambda \sum_{i=1}^{n} \mathbf{Z}_{i}^{\top} \mathbf{F}_{i} \mathbf{M}_{i} \operatorname{Cov}^{-1}\left(\varepsilon_{i}\right) \varepsilon, \\
s_{i} & =\sum_{i=1}^{n} \sum_{j=1}^{t} \frac{w_{i j}\left\{\cos \left(y_{i j}-\mu_{i j}\right)-\mathcal{A}_{1}\left(w_{i j} \lambda\right)\right\}}{\mathbf{1}_{t}^{\top} \operatorname{Cov}\left(\varepsilon_{i}\right) \mathbf{1}_{t}},
\end{aligned}
$$

e, como $E\left(\mathbf{d}_{i}\right)=\delta\left(\mathbf{N}_{i}\right)$, tem-se que $\operatorname{Cov}\left(\varepsilon_{i}\right)=\operatorname{Cov}\left(\mathbf{d}_{i}\right)$.

Resultados adicionais relativos à aplicação da função (3.25) a dados circulares estão descritos nesta seção.

\section{Função de estimação ótima em $\mathcal{L}$}

Suponha, sem perda de generalidade que $y_{i j} \sim \mathcal{M}\left(\mu_{i j} ; \lambda_{i j}\right)$, com

$$
\lambda_{i j}=q\left(\xi_{i j}\right) .
$$

A partir de (3.24), chega-se que a função de estimação ótima na classe $\mathcal{L}$, gerada a partir de $\left(u_{i}, \varepsilon_{i}\right)^{\top}$, é dada por:

$$
\mathbf{\Upsilon}_{n}^{*}(\boldsymbol{\theta})=\sum_{i=1}^{n}\left(\begin{array}{cc}
\mathbf{X}_{i}^{\top} \mathbf{H}_{i} \mathbf{N}_{i} & 0 \\
0 & \mathbf{Z}_{i}^{\top} \mathbf{F}_{i} \mathbf{M}_{i}
\end{array}\right)\left\{\begin{array}{cc}
\mathbf{W}_{i} & E\left(\mathbf{u}_{i} \varepsilon_{i}^{\top}\right) \\
E\left(\varepsilon_{i} \mathbf{u}_{i}^{\top}\right) & \operatorname{Cov}\left(\varepsilon_{i}\right)
\end{array}\right\}^{-1}\left(\begin{array}{c}
\mathbf{u}_{i} \\
\varepsilon_{i}
\end{array}\right)
$$


Definindo-se, a partir de propriedades de inversa de matrizes particionadas ${ }^{6}$, as matrizes:

$$
\begin{gathered}
\overline{\mathbf{W}}_{11 i}=\left\{\mathbf{W}_{i}-E\left(\mathbf{u}_{i} \varepsilon_{i}^{\top}\right) \operatorname{Cov}^{-1}\left(\varepsilon_{i}\right) E\left(\varepsilon_{i} \mathbf{u}_{i}^{\top}\right)\right\}^{-1}, \\
\overline{\mathbf{W}}_{22 i}=\left\{\mathbf{W}_{i}-E\left(\varepsilon_{i} \mathbf{u}_{i}^{\top}\right) \mathbf{W}_{i}^{-1} E\left(\mathbf{u}_{i} \varepsilon_{i}^{\top}\right)\right\}^{-1} \\
\overline{\mathbf{W}}_{12 i}=-\mathbf{W}_{i}^{-1} E\left(\mathbf{u}_{i} \varepsilon_{i}^{\top}\right)\left\{\mathbf{W}_{i}-E\left(\varepsilon_{i} \mathbf{u}_{i}^{\top}\right) \mathbf{W}_{i}^{-1} E\left(\mathbf{u}_{i} \varepsilon_{i}^{\top}\right)\right\}^{-1}
\end{gathered}
$$

$\mathrm{e}$

$$
\overline{\mathbf{W}}_{21 i}=-\operatorname{Cov}^{-1}\left(\varepsilon_{i}\right) E\left(\varepsilon_{i} \mathbf{u}_{i}^{\top}\right)\left\{\mathbf{W}_{i}-E\left(\mathbf{u}_{i} \varepsilon_{i}^{\top}\right) \operatorname{Cov}^{-1}\left(\varepsilon_{i}\right) E\left(\varepsilon_{i} \mathbf{u}_{i}^{\top}\right)\right\}^{-1},
$$

temos que a função de estimação ótima em $\mathcal{L}$ pode ser reescrita como

$$
\mathbf{\Upsilon}_{n}^{*}(\boldsymbol{\theta})=\sum_{i=1}^{n}\left\{\begin{array}{l}
\mathbf{X}_{i}^{\top} \mathbf{H}_{i} \mathbf{N}_{i}\left(\overline{\mathbf{W}}_{11 i} \mathbf{u}_{i}+\overline{\mathbf{W}}_{12 i} \varepsilon_{i}\right) \\
\mathbf{Z}_{i}^{\top} \mathbf{F}_{i} \mathbf{M}_{i}\left(\overline{\mathbf{W}}_{21 i} \mathbf{u}_{i}+\overline{\mathbf{W}}_{22 i} \varepsilon_{i}\right)
\end{array}\right\}
$$

A partir dessas expressões e utilizando-se o fato de $\mathbf{u}_{i}$ e $\varepsilon_{i}$ terem vetor média zero, vem que sob as condições do Corolário 3 a matriz de covariância assintótica de $\sqrt{n}\left(\hat{\boldsymbol{\theta}}_{n}-\boldsymbol{\theta}\right)$, onde $\hat{\boldsymbol{\theta}}_{n}$ é a raiz da função de estimação anterior, é dada por

$$
\mathbf{J}^{-1}=n \mathbf{S}^{-1}(\boldsymbol{\theta}) \mathbf{V}(\boldsymbol{\theta}) \mathbf{S}^{-\top}(\boldsymbol{\theta}),
$$

com

$$
\begin{gathered}
\mathbf{S}(\boldsymbol{\theta})=\left(\begin{array}{ll}
\mathbf{S}_{11} & \mathbf{S}_{12} \\
\mathbf{S}_{12}^{\top} & \mathbf{S}_{22}
\end{array}\right), \\
\mathbf{S}_{11}=\sum_{i=1}^{n} \mathbf{X}_{i}^{\top} \mathbf{H}_{i} \mathbf{N}_{i} \overline{\mathbf{W}}_{11} \mathbf{N}_{i} \mathbf{H}_{i} \mathbf{X}_{i}, \\
\mathbf{S}_{22}=\sum_{i=1}^{n} \mathbf{Z}_{i}^{\top} \mathbf{F}_{i} \mathbf{M}_{i} \overline{\mathbf{W}}_{22} \mathbf{M}_{i} \mathbf{F}_{i} \mathbf{Z}_{i}, \\
\mathbf{S}_{12}=\sum_{i=1}^{n} \mathbf{X}_{i}^{\top} \mathbf{H}_{i} \mathbf{N}_{i} \overline{\mathbf{W}}_{12} \mathbf{M}_{i} \mathbf{F}_{i} \mathbf{Z}_{i}, \\
\mathbf{V}(\theta)=\left(\begin{array}{ll}
\mathbf{V}_{11} & \mathbf{V}_{12} \\
\mathbf{V}_{12}^{\top} & \mathbf{V}_{22}
\end{array}\right),
\end{gathered}
$$

\footnotetext{
${ }^{6}$ ver Graybill (1969), por exemplo
} 


$$
\begin{gathered}
\mathbf{V}_{11}=\sum_{i=1}^{n} \mathbf{X}_{i}^{\top} \mathbf{H}_{i} \mathbf{N}_{i} \operatorname{Cov}\left(\overline{\mathbf{W}}_{11 i} \mathbf{u}_{i}+\overline{\mathbf{W}}_{12 i} \varepsilon_{i}\right) \mathbf{N}_{i} \mathbf{H}_{i} \mathbf{X}_{i} \\
\mathbf{V}_{22}=\sum_{i=1}^{n} \mathbf{Z}_{i}^{\top} \mathbf{F}_{i} \mathbf{M}_{i} \operatorname{Cov}\left(\overline{\mathbf{W}}_{21 i} \mathbf{u}_{i}+\overline{\mathbf{W}}_{22 i} \varepsilon_{i}\right) \mathbf{M}_{i} \mathbf{F}_{i} \mathbf{Z}_{i} \\
\mathbf{V}_{12}=\sum_{i=1}^{n} \mathbf{X}_{i}^{\top} \mathbf{H}_{i} \mathbf{N}_{i} E\left\{\left(\overline{\mathbf{W}}_{11 i} \mathbf{u}_{i}+\overline{\mathbf{W}}_{12 i} \varepsilon_{i}\right)\left(\overline{\mathbf{W}}_{21 i} \mathbf{u}_{i}+\overline{\mathbf{W}}_{22 i} \varepsilon_{i}\right)^{\top}\right\} \mathbf{M}_{i} \mathbf{F}_{i} \mathbf{Z}_{i}
\end{gathered}
$$

No caso usual, onde as matrizes de covariâncias envolvidas em (3.30) são desconhecidas, elas podem ser substituídas, conforme já salientado, por estimativas que satisfazem o Teorema 7 . Os estimadores resultantes dessa nova função de estimação são, sob condições gerais, assintoticamente normais com matriz de covariância assintótica dacla por $\overline{\mathbf{J}}^{-1}$. Essa matriz pode ser consistentemente estimada por

$$
\hat{\overline{\mathbf{J}}}^{-1}=n \mathbf{S}^{-1}\left(\hat{\boldsymbol{\theta}}_{n}\right)\left\{\sum_{i=1}^{n} \boldsymbol{v}_{i}^{*}\left(\hat{\boldsymbol{\theta}}_{n}\right) \boldsymbol{v}_{i}^{* \mathrm{~T}}\left(\hat{\boldsymbol{\theta}}_{n}\right)\right\} \mathbf{S}\left(\hat{\boldsymbol{\theta}}_{n}^{-\mathrm{T}}\right) .
$$

\section{Adaptação para o modelo (1.5)}

Fisher (1993) apresenta modelos de regressão para os parâmetros de posição e concentração de uma distribuição von Mises. Nesses modelos, a partir da versão (1.3) da função densidade de probabilidades da von Mises, considera-se $y_{i j} \sim \mathcal{M}\left(\mu_{i j} ; \lambda_{i j}\right)$, com

$$
\begin{aligned}
\mu_{i j} & =\beta_{0}+g\left(\eta_{i j}\right) \\
\lambda_{i j} & =q\left(\xi_{i j}\right) .
\end{aligned}
$$

Como exemplo de função $q$ tem-se

$$
\lambda_{i j}=\exp \left(\gamma_{0}+\sum_{l=1}^{m} z_{i j l} \gamma_{l}\right) .
$$

Para o modelo (3.31), considerando-se $\theta=\left(\beta_{0}, \boldsymbol{\beta}, \boldsymbol{\gamma}\right)$, tem-se que as funções de estimação de independência e a baseada em (3.26) são dadas, respectivamente, por

$$
\Upsilon_{n}^{I}(\theta)=\sum_{i=1}^{n}\left(\begin{array}{c}
\mathbf{1}_{t}^{\top} \Delta\left(\boldsymbol{\lambda}_{i}\right) \mathbf{u}_{i} \\
\mathbf{X}_{i}^{\top} \mathbf{H}_{i} \Delta\left(\boldsymbol{\lambda}_{i}\right) \mathbf{u}_{i} \\
\mathbf{Z}_{i} \mathbf{F}_{i} \varepsilon_{i}
\end{array}\right)
$$


onde $\boldsymbol{\lambda}_{i}=\left(\lambda_{i 1}, \lambda_{i 2}, \ldots, \lambda_{i t}\right) \mathrm{e}$

$$
\Upsilon_{n}(\theta)=\sum_{i=1}^{n}\left(\begin{array}{c}
\delta^{\top}\left(\mathbf{N}_{i}\right) \mathbf{W}_{i}^{-1} \mathbf{u}_{i} \\
\mathbf{X}_{i}^{\top} \mathbf{H}_{i} \mathbf{N}_{i} \mathbf{W}_{i}^{-1} \mathbf{u}_{i} \\
\mathbf{Z}_{i} \mathbf{F}_{i}\left\{\varepsilon_{i}\right\}
\end{array}\right) .
$$

Para a função $\Upsilon_{n}(\theta)$ tem-se também

$$
\mathbf{S}=\lim _{n \rightarrow \infty} \frac{1}{n}\left(\begin{array}{ccc}
s_{011} & \mathrm{~S}_{012}^{\top} & 0 \\
\mathbf{S}_{012} & \mathbf{S}_{11} & 0 \\
0 & 0 & \mathbf{S}_{22}
\end{array}\right)
$$

$\mathrm{e}$

$$
\mathbf{V}=\lim _{n \rightarrow \infty} \frac{1}{n}\left(\begin{array}{lll}
v_{011} & \mathbf{v}_{012}^{\top} & \mathbf{v}_{013}^{\top} \\
\mathbf{V}_{012} & \mathbf{V}_{11} & \mathbf{V}_{12} \\
\mathbf{v}_{013} & \mathbf{V}_{12}^{\top} & \mathbf{V}_{22}
\end{array}\right)
$$

onde

$$
\begin{gathered}
s_{011}=-\sum_{i=1}^{n} \delta^{\top}\left(\mathbf{N}_{i}\right) \mathbf{W}_{i}^{-1} \delta\left(\mathbf{N}_{i}\right), \\
\mathbf{s}_{012}=-\sum_{i=1}^{n} \mathbf{X}_{i}^{\top} \mathbf{H}_{i} \mathbf{N}_{i} \mathbf{W}_{i}^{-1} \delta\left(\mathbf{N}_{i}\right), \\
\mathbf{S}_{11}=-\sum_{i=1}^{n} \mathbf{X}_{i}^{\top} \mathbf{H}_{i} \mathbf{N}_{i} \mathbf{W}_{i}^{-1} \mathbf{N}_{i} \mathbf{H}_{i} \mathbf{X}_{i} \\
\mathbf{S}_{22}=-\sum_{i=1}^{n} \mathbf{Z}_{i}^{\top} \mathbf{F}_{i} \Delta\left(\dot{\mathbf{N}}_{i}\right) \mathbf{F}_{i} \mathbf{Z}_{i}, \\
v_{011}=\sum_{i=1}^{n} \delta^{\top}\left(\mathbf{N}_{i}\right) \mathbf{W}_{i}^{-1} \operatorname{Cov}\left(\mathbf{u}_{i}\right) \mathbf{W}_{i}^{-1} \delta\left(\mathbf{N}_{i}\right) \\
\mathbf{v}_{012}=\sum_{i=1}^{n} \delta^{\top}\left(\mathbf{N}_{i}\right) \mathbf{W}_{i}^{-1} \operatorname{Cov}\left(\mathbf{u}_{i}\right) \mathbf{W}_{i}^{-1} \mathbf{N}_{i} \mathbf{H}_{i} \mathbf{X}_{i}, \\
\mathbf{v}_{013}=\sum_{i=1}^{n} \mathbf{Z}_{i}^{\top} \mathbf{F}_{i} E\left(\mathbf{u}_{i} \mathbf{d}_{i}^{\top}\right) \mathbf{W}_{i}^{-1} \delta\left(\mathbf{N}_{i}\right) \\
\mathbf{V}_{11}=\sum_{i=1}^{n} \mathbf{X}_{i} \mathbf{H}_{i} \mathbf{N}_{i} \mathbf{W}_{i}^{-1} \operatorname{Cov}\left(\mathbf{u}_{i}\right) \mathbf{W}_{i}^{-1} \mathbf{N}_{i} \mathbf{H}_{i} \mathbf{X}_{i}, \\
\mathbf{V}_{12}=\sum_{i=1}^{n} \mathbf{X}_{i}^{\top} \mathbf{H}_{i} \mathbf{N}_{i} \mathbf{W}_{i}^{-1} E\left(\mathbf{u}_{i} \mathrm{~d}_{i}\right) \mathbf{F}_{i} \mathbf{Z}_{i}
\end{gathered}
$$


$\mathrm{e}$

$$
\mathbf{V}_{22}=\sum_{i=1}^{n} \mathbf{Z}_{i}^{\top} \mathbf{F}_{i} \operatorname{Cov}\left(\mathbf{d}_{i}\right) \mathbf{F}_{i} \mathbf{Z}_{i}
$$

A fim de facilitar o algoritmo de estimaçào, igualando-se a zero a primeira linha da matriz $\Upsilon_{n}(\theta)$ tem-se

$$
\begin{aligned}
\sum_{i=1}^{n} \sum_{j=1}^{t} \sum_{k=1}^{t} \mathcal{A}_{1}\left(\lambda_{i j}\right) \omega_{i j k} \operatorname{sen}\left\{y_{i k}-g\left(\eta_{i k}\right)\right\} \\
=\sum_{i=1}^{n} \sum_{j=1}^{t} \sum_{k=1}^{t} \mathcal{A}_{1}\left(\lambda_{i j}\right) \omega_{i j k} \cos \left\{y_{i k}-g\left(\eta_{i k}\right)\right\} \tan \left(\beta_{0}\right),
\end{aligned}
$$

onde $\omega_{i j k}$ corresponde à componente $(j, k)$ de $\mathbf{W}_{i}^{-1}$ e, definindo-se

$$
\begin{aligned}
& S=\sum_{i=1}^{n} \sum_{j=1}^{t} \sum_{k=1}^{t} \mathcal{A}_{1}\left(\lambda_{i j}\right) \omega_{i j k} \operatorname{sen}\left\{y_{i k}-g\left(\eta_{i k}\right)\right\} \\
& C=\sum_{i=1}^{n} \sum_{j=1}^{t} \sum_{k=1}^{t} \mathcal{A}_{1}\left(\lambda_{i j}\right) \omega_{i j k} \cos \left\{y_{i k}-g\left(\eta_{i k}\right)\right\},
\end{aligned}
$$

tem-se

$$
\hat{\beta}_{0}= \begin{cases}\arctan (S / C), & \text { se } S \geq 0 \text { e } C>0 \\ \arctan (S / C)+\pi, & \text { se } C<0 \\ \arctan (S / C)+2 \pi, & \text { se } S<0 \text { e } C>0\end{cases}
$$




\section{Capítulo 4}

\section{Normal arqueada multivariada e simulações}

Neste capítulo é apresentada uma distribuição de probabilidades multivariada para variáveis circulares baseada na distribuição normal arqueada e posteriormente são desenvolvidos estudos de simulação, visando avaliar o desempenho de algumas funções de estimação sugeridas nesta tese e do estimador de máxima verossimilhança baseado na normal arqueada multivariada.

\subsection{Distribuição normal arqueada multivaria- da}

Neste tópico pretende-se apresentar uma modelagem alternativa para dados circulares longitudinais (multivariados). A idéia principal é assumir que as distribuições marginais dos vetores resposta seguem uma distribuição normal arqueada e definir uma distribuição normal arqueada multivariada de tal sorte que seja possível a estimação dos parâmetros através do método de máxima verossimilhança.

Seja $\mathbf{Z}=\left(Z_{1}, \ldots, Z_{t}\right)^{\top} \sim \mathcal{N}_{t}(\boldsymbol{\mu} ; \boldsymbol{\Sigma})$ uma distribuição normal $t$-variada com vetor média $\boldsymbol{\mu}=\left(\mu_{1}, \ldots, \mu_{t}\right)^{\top}$ e matriz de covariâncias $\Sigma=\left[\sigma_{j k}\right]$, $j, k=1,2, \ldots, t$. Definindo $\mathrm{y}=\left(y_{1}, \ldots, y_{t}\right)^{\top}$ um vetor aleatório com $y_{j}=$ $Z_{j}(\bmod 2 \pi)$, então y é dita seguir uma distribuição normal arqueada $t$-variada com parâmetros $\mu$ e $\Sigma$, cuja notação é y $\sim \mathcal{N} \mathcal{E}_{t}(\mu: \Sigma)$.

Proposição 3 . Seja y $\sim \mathcal{N} \mathcal{E}_{t}(\boldsymbol{\mu} ; \boldsymbol{\Sigma})$, conforme definida anteriormente. 
Então sua funçâo densidade de probabilidades é dada por

$$
\begin{aligned}
p(\mathrm{y} ; \boldsymbol{\mu}, \boldsymbol{\Sigma}) & =\frac{1}{(2 \pi)^{t / 2}|\Sigma|^{1 / 2}} \\
& \sum_{\mathrm{k} \in \mathcal{Z}^{t}} \exp \left\{-\frac{1}{2}(\mathrm{y}+2 \mathrm{k} \pi-\boldsymbol{\mu})^{\top} \boldsymbol{\Sigma}^{-1}(\mathrm{y}+2 \mathrm{k} \pi-\boldsymbol{\mu})\right\} .
\end{aligned}
$$

Prova: Sejam $F_{\mathbf{y}}$ a função distribuição acumulada de $\mathbf{y}, f$ a função densidade de probabilidades de uma normal $t$-variada com média $\boldsymbol{\mu}$ e matriz de covariâncias $\Sigma, \mathbf{k}=\left(k_{1}, \ldots, k_{t}\right)^{\top}$ e $\mathbf{c}=\left(c_{1}, \ldots, c_{t}\right)^{\top}, c_{j} \in[0,2 \pi)$. Tern-se, então que

$$
\begin{aligned}
F_{\mathbf{y}}(\mathbf{c}) & =P\left(0 \leq y_{1} \leq c_{1}, \ldots, 0 \leq y_{t} \leq c_{t}\right) \\
& =\sum_{\mathbf{k} \in \mathcal{Z}^{t}} P\left(2 k_{1} \pi \leq z_{1} \leq 2 k_{1} \pi+c_{1}, \ldots, 2 k_{t} \pi \leq z_{t} \leq 2 k_{t} \pi+c_{t}\right) \\
& =\sum_{\mathbf{k} \in \mathcal{Z}^{t}} \int_{2 k_{1}}^{2 k_{1} \pi+c_{1}} \cdots \int_{2 k_{t}}^{2 k_{t} \pi+c_{t}} f\left(x_{1}, \ldots, x_{t}\right) d x_{t} \ldots d x_{1} \\
& =\sum_{\mathbf{k} \in \mathcal{Z}^{t}} \int_{0}^{c_{1}} \cdots \int_{0}^{c_{t}} f\left(x_{1}+2 k_{1}, \ldots, x_{t}+2 k_{t}\right) d x_{t} \ldots d x_{1} .
\end{aligned}
$$

Como essa integral é convergente e $f$ é positiva, tem-se que

$$
F_{\mathbf{y}}(\mathbf{c})=\int_{0}^{c_{1}} \cdots \int_{0}^{c_{t}}\left\{\sum_{\mathbf{k} \in \mathcal{Z}^{t}} f\left(x_{1}+2 k_{1}, \ldots, x_{t}+2 k_{t}\right)\right\} d x_{t} \ldots d x_{1} .
$$

Portanto, segue que

$$
p(\mathbf{y} ; \boldsymbol{\mu}, \boldsymbol{\Sigma})=\sum_{\mathbf{k} \in \mathcal{Z}^{t}} f\left(x_{1}+2 k_{1}, \ldots, x_{t}+2 k_{t}\right),
$$

o que conclui a demonstração. o

Uma propriedade importante dessa distribuição é que suas marginais são distribuições normais arqueadas univariadas, com média circular $\mu_{j}$ e parâmetro de dispersão $\sigma_{j j}, j=1,2, \ldots, t$.

Para auxiliar a interpretação dos parâmetros de dependência, podemos escrever $\sigma_{j k}, j \neq k$, como função do coeficiente de correlação toroidal entre $y_{j}$ e $y_{k}$. Temos então, a partir de (1.10), que

$$
\rho_{T_{\jmath k}}=\frac{\operatorname{senh}\left(2 \sigma_{j k}\right)}{\left\{\operatorname{senh}\left(2 \sigma_{j j}\right) \operatorname{senh}\left(2 \sigma_{k k}\right)\right\}^{1 / 2}} .
$$




\subsubsection{Modelos para a análise de dados longitudinais}

Sejam $\mathbf{y}_{i}, i=1,2 \ldots, n$, vetores normais arqueados $t$-dimensionais com vetor média circular $\mu_{i}=\left(\mu_{i 1}, \ldots, \mu_{i t}\right)^{\top}$ e matriz de parâmetros de dependência $\Sigma$. Considere a existência de $p$ covariáveis e $\mu_{i j}$ modelado como na Seçâo 3.1. Para simplificar futuras expressões, defina

$$
e_{i \mathbf{k}}=\exp \left\{-\frac{1}{2}\left(\mathrm{y}_{i}+2 \mathbf{k}_{i} \pi-\boldsymbol{\mu}_{i}\right)^{\top} \boldsymbol{\Sigma}^{-1}\left(\mathrm{y}_{i}+2 \mathbf{k}_{i} \pi-\boldsymbol{\mu}_{i}\right)\right\}
$$

e $e_{i}=\sum_{\mathbf{k}_{i} \in \mathcal{Z}^{t}} e_{i \mathbf{k}}$. A função de log-verossimilhança associada aos parâmetros, omitindo-se a parcela constante, é dada por

$$
L(\boldsymbol{\beta}, \boldsymbol{\Sigma})=-\frac{n}{2} \log |\boldsymbol{\Sigma}|+\sum_{i=1}^{n} \log e_{i} .
$$

Maximizando-se $L$ em $\boldsymbol{\beta}$ e $\boldsymbol{\Sigma}$, tem-se que os estimadores de máxima verossimilhança desses parâmetros são dados pelas raízes de

$$
\begin{gathered}
\frac{\partial L}{\partial \boldsymbol{\beta}}(\boldsymbol{\beta}, \boldsymbol{\Sigma})=\sum_{i=1}^{n} \sum_{\mathbf{k}_{i} \in \mathcal{Z}^{t}} \frac{e_{i \mathbf{k}}}{e_{i}} \mathbf{X}_{i}^{\top} \mathbf{H}_{i} \boldsymbol{\Sigma}^{-1}\left(\mathbf{y}_{i}+2 \mathbf{k}_{i} \pi-\boldsymbol{\mu}_{i}\right) \\
\frac{\partial L}{\partial \boldsymbol{\Sigma}}(\boldsymbol{\beta}, \boldsymbol{\Sigma})=-\frac{n}{2}\left[2 \boldsymbol{\Sigma}^{-1}-\Delta\left\{\delta\left(\boldsymbol{\Sigma}^{-1}\right)\right\}\right] \\
+\sum_{i=1}^{n} \sum_{\mathbf{k}_{i} \in \mathcal{Z}^{t}} \frac{e_{i \mathbf{k}}}{e_{i}} \boldsymbol{\Sigma}^{-1}\left(\mathbf{y}_{i}+2 \mathbf{k}_{i} \pi-\boldsymbol{\mu}_{i}\right)\left(\mathbf{y}_{i}+2 \mathbf{k}_{i} \pi-\boldsymbol{\mu}_{i}\right)^{\top} \boldsymbol{\Sigma}^{-1} \\
-\frac{1}{2} \sum_{i=1}^{n} \sum_{\mathbf{k}_{i} \in \mathcal{Z}^{t}} \frac{e_{i \mathbf{k}}}{e_{i}} \Delta\left[\delta\left\{\boldsymbol{\Sigma}^{-1}\left(\mathbf{y}_{i}+2 \mathbf{k}_{i} \pi-\boldsymbol{\mu}_{i}\right)\left(\mathbf{y}_{i}+2 \mathrm{k}_{i} \pi-\boldsymbol{\mu}_{i}\right)^{\top} \boldsymbol{\Sigma}^{-1}\right\}\right] .
\end{gathered}
$$

\subsubsection{Algoritmos de estimação}

Os algoritmos de estimação convencionais utilizam a segunda derivada do logaritmo da função de verossimilhança em relação aos parâmetros. No caso da função (4.1), essas derivadas têm uma forma bastante complicada, como pode ser deduzido da observação de (4.2) e (4.3), principalmente quando a dimensão de $\boldsymbol{\beta}$ é alta. Nesses casos, aconselha-se o uso de métodos alternativos de estimação, tais como o método de quase-Newton (ver Chambers, 1977 
ou Nash, 1990, por exemplo), onde a segunda derivada da função de verossimilhança é avaliada numericamente.

Outra dificuldade do processo de estimação diz respeito ao cálculo das séries $e_{i}, \sum_{\mathbf{k}_{\mathbf{i}} \in Z^{t}} e_{i \mathbf{k}} \mathbf{k}_{i}$ e $\sum_{\mathbf{k}_{i} \in \mathcal{Z}^{t}} e_{i \mathbf{k}} \mathbf{k}_{i} \mathbf{k}_{i}^{\top}$, que aparecem em (4.2) e (4.3). A fim de estimá-las, pode-se por exemplo aproximar o valor da série por uma soma finita de termos, partindo-se do fato que a soma das parcelas para valores de $\mathbf{k}_{\boldsymbol{i}}$ distantes de $\boldsymbol{\mu}_{\boldsymbol{i}}$ tende a diminuir, conforme essa distância aumenta. Outro procedimento seria utilizar o método de Monte Carlo (ver Sobol(1975), por exemplo) para avaliar as séries. No Apêndice D essas opções são detalhadas.

\subsubsection{Estimação dos parâmetros de regressão via fun- ções de estimação}

Os parâmetros de regressão de um modelo normal arqueado multivariado também podem ser estimados pela metodologia desenvolvida nas Seções 3.1 e 3.3. Os estimadores assim obtidos têm as mesmas propriedades daqueles obtidos naquelas seções. Isso se verifica pois a validade das propriedades depende basicamente da propriedade $E\left(\mathbf{u}_{i}\right)=0$, onde as componentes de $\mathbf{u}_{i}$ são dadas por sen $\left(y_{i j}-\mu_{i j}\right)$ que possuem esperança nula, mesmo sob a hipótese de distribuição marginal normal arqueada.

No caso da distribuição normal arqueada multivariada, é possível estabelecer uma relação direta entre os parâmetros de dependência da distribuição e os que formam a matriz $\mathbf{W}_{i}=\operatorname{Cov}\left(\mathbf{u}_{i}\right)$. Temos que

$$
\operatorname{Cov}\left\{\operatorname{sen}\left(y_{i j}-\mu_{i j}\right), \operatorname{sen}\left(y_{i k}-\mu_{i k}\right)\right\}=\exp \left\{-\frac{1}{2}\left(\sigma_{j j}+\sigma_{k k}\right)\right\} \operatorname{senh}\left(\sigma_{j k}\right) .
$$

No caso da matriz de correlação, temos

$$
\operatorname{Corr}\left\{\operatorname{sen}\left(y_{i j}-\mu_{i j}\right), \operatorname{sen}\left(y_{i k}-\mu_{i k}\right)\right\}=\frac{\operatorname{senh}\left(\sigma_{j k}\right)}{\left\{\operatorname{senh}\left(\sigma_{j j}\right) \operatorname{senh}\left(\sigma_{k k}\right)\right\}^{1 / 2}} .
$$

\subsection{Simulações}

Nesta seção são apresentados estudos de simulação realizados para comparar os resultados desenvolvidos nos Capítulos 3 e 4 . Os estudos estão divididos em duas partes: na primeira pretende-se comparar, em diferentes situações experimentais, as estimativas obtidas através das funções de estimação (3.1) 
e (3.7) e, na segunda, pretende-se comparar os estimadores definidos como raiz de (3.1) e (3.7) com os estimadores de máxima verossimilhança, quando é suposto que os dados seguem uma distribuição normal arqueada multivariada.

Em todos os estudos, os dados foram gerados através de uma distribuição normal arqueada multivariada. Cabe lembrar que apesar da metodologia ter sido desenvolvida para modelos de dispersão, sua utilização não se restringe a essa classe. Em particular, para dados circulares o método baseado na distribuição von Mises é aplicável à normal arqueada. Optou-se por essa distribuição pela facilidade de sua geração e por não existir uma distribuição multivariada com marginais von Mises que seja considerada canônica.

As simulaçōes foram realizadas através de programas computacionais escritos na linguagem $\mathrm{C}$ e S-plus. Parte do estudo foi desenvolvido em estações de trabalho Sun e parte no super-computador Cray do Laboratório de Ciência de Computação Avançada do Centro de Computação Eletrônica da Universidade de São Paulo.

\subsubsection{Geração da normal arqueada multivariada}

O procedimento de geração consiste em inicialmente gerar observações $\mathbf{z}$ de uma distribuição normal $t$-variada com média 0 e matriz de covariâncias $\boldsymbol{\Sigma}$. A seguir, obtem-se números pseudo-aleatórios de uma normal arqueada $t$ - variada com média circular $\boldsymbol{\mu}$ e matriz de parâmetros de dependência $\boldsymbol{\Sigma}$ efetuando-se $\mathrm{y}=(\mathrm{z} \bmod 2 \pi)+\boldsymbol{\mu}$.

\subsubsection{Estudo 1}

Neste estudo, cada simulação é composta por $n(n=20,50,100)$ observações geradas a partir de uma distribuição normal arqueada $t$-multivariada $(t=5,12)$, onde a média circular é afetada por uma única covariável, gerada a partir de um distribuição normal padrão. Após a geração das covariáveis, seus valores forarn centrados a fim de aumentar a eficiência do algoritmo de estimação.

A função de ligação utilizada para a média é

$$
\mu_{i j}=\beta_{0}+2 \arctan \left(\beta x_{i j}\right)
$$

$\operatorname{com} \beta_{0}=1$ e $\beta=0,2$. 
Os parâmetros de variabilidade marginais são os mesmos para todas as componentes do vetor resposta, $\sigma^{2}$. Utilizando a relação (1.4) entre o parâmetro de dispersão da normal arqueada e o de concentração $\lambda$, de uma von Mises, as simulações são realizadas $\operatorname{com} \lambda=1,2$ e 7 . A escolha desses valores justifica-se uma vez que o valor 7 é bastante elevado e sob essa circunstância a von Mises pode ser aproximada por uma distribuição normal; os valores 2 e 1 são limites utilizados na literatura especializada (ver Fisher, 1993, Batschelet, 1981 e Mardia, 1972, por exemplo), a partir dos quais recomenda-se a utilização de uma abordagem paramétrica (se $\lambda$ for superior ao limite) ou não paramétrica (caso contrário).

É suposta uma estrutura auto-regressiva de ordem 1 para a matriz $\Sigma$. São geradas observações utilizando-se auto-correlações $(\rho)$ 0,0, 0,5 e 0,9.

Em cada simulação, são obtidas estimativas utilizando-se duas funções de estimação: a primeira obtida ao ignorar-se a estrutura de correlação existente, EEI, (baseada na função (3.1)) e a segunda, EEG, onde essa estrutura é considerada desconhecida, o que obriga a estimação do vetor pasamétrico $\alpha$ (baseada na função (3.23)).

Apesar de $\boldsymbol{\Sigma}$ possuir uma estrutura bem definida, optou-se por desconsiderar esse fato na estimação de $\alpha$, uma vez que na prática desconhece-se a estrutura geradora dos dados. Desse modo, todas as componentes da matriz de correlação serão estimadas independentemente das demais.

Para cada combinação de $n, t, \lambda$ e $\rho, 1000$ simulações foram conduzidas.

Já para o estimador de máxima verossimilhança, devido a limitações computacionais, são realizadas apenas 200 simulações por combinação e, mais ainda, a matriz $\Sigma$ é suposta conhecida.

\section{Algoritmo de estimação}

Por sua relativa simplicidade, optou-se pela utilização do algoritmo modificado de Newton. Sejam $w_{j k}$ as componentes de $\mathbf{W}^{-1}, \beta_{0}^{(l)}$ e $\beta^{(l)}$ as estimativas de $\beta_{0}$ e $\beta$ no $l$-ésimo passo do processo iterativo. Então

$$
\begin{aligned}
S & =\sum_{i=1}^{n} \sum_{j=1}^{t} \sum_{k=1}^{t} w_{j k} \operatorname{sen}\left\{y_{i k}-2 \arctan \left(\beta x_{i k}\right)\right\}, \\
C & =\sum_{i=1}^{n} \sum_{j=1}^{t} \sum_{k=1}^{t} w_{j k} \cos \left\{y_{i k}-2 \arctan \left(\beta x_{i k}\right)\right\} \\
R^{2} & =\left(C^{2}+S^{2}\right)^{1 / 2},
\end{aligned}
$$


e as equações de estimação utilizadas são:

$$
\beta_{0}^{(l)}= \begin{cases}\arctan (S / C), & \text { se } S \geq 0 \text { e } C>0 \\ \arctan (S / C)+\pi, & \text { se } C<0 \\ \arctan (S / C)+2 \pi, & \text { se } S<0 \text { e } C>0\end{cases}
$$

e

$$
\beta^{(l+1)}=\beta^{(l)}+\frac{1}{\mathcal{A}(\lambda)} \frac{\sum_{i=1}^{n} \sum_{j=1}^{t} \sum_{k=1}^{t} x_{i j} h_{i j} w_{j k} \operatorname{sen}\left(y_{i k}-\mu_{i k}\right)}{\sum_{i=1}^{n} \sum_{j=1}^{t} \sum_{k=1}^{t} x_{i j} h_{i j} w_{j k} h_{i k} x_{i k}},
$$

onde $\bar{R}=R / n=\mathcal{A}(\lambda), h_{i j}=2 /\left\{1+\left(\beta x_{i j}\right)^{2}\right\}$. No caso das EEI, imponha $w_{j k}=0$, se $j \neq k$ e 1 , caso $j=k$.

\section{Regra de parada e valor inicial}

Como o objetivo é avaliar as estimativas obtidas através das funções de estimação, optou-se por utilizar 1 e 0,2 como valores iniciais para $\beta_{0}$ e $\beta$.

Os algoritmos de estimação foram considerados convergentes quando o máximo das diferenças entre iterações consecutivas e o máximo da diferença entre os componentes das funções de estimação em duas iterações consecutivas fosse inferior a $10^{-5}$.

\section{Desempenho dos algoritmos de estimação}

Não houve problemas de convergência com o algoritmo de estimação para EEI.

O algoritmo de estimação para EEG apresentou uma performance bastante satisfatória na maioria das situações simuladas. Dificuldades surgiram principalmente para $n=20$ e $\lambda=1$. Trata-se do caso onde a variabilidade dos dados é muito alta e o tamanho amostral pequeno. No caso $n=20$, $\lambda=1, t=5$, para $\rho=0,9$ e 0,5 houve até cinco casos (em mil) onde o algoritmo não convergiu até a iteração 600: uma das causas prováveis desses problemas pode estar na escolha do valor inicial do processo iterativo. Para $n=20$ e $t=12$, problemas surgiram $\operatorname{com} \lambda=1$ e 2 , para $\rho=0,5$ e 0,9 : o número de situações onde não houve convergência variou em torno de 9 (em mil), quando $\rho=0,5, \lambda=2$, até aproximadamente 200 (em mil) no caso em que $\rho=0,9$ e $\lambda=1$. Note que nessa situação o tamanho amostral 
é muito pequeno em relação ao número de parâmetros de perturbação (as covariâncias) que devem ser estimados e talvez essa seja a causa do problema. Isso está de acordo com o estudo de simulação de Lipsitz et al. (1994) descrito na Seção 3.2.

\section{Avaliação dos resultados}

Para a comparação do desempenho das funções de estimação na estimação do parâmetro de regressão $\beta$, calculou-se duas medidas:

i. o erro absoluto médio amostral percentual, definido por

$$
\mathrm{EAM}_{j}=\sum_{i=1}^{1000} \frac{\left|\hat{\beta}_{i j}-0,2\right| / 1000}{0,2}, j=1,2
$$

onde $\hat{\beta}_{i j}$ corresponde à $i$-ésima estimativa do parâmetro $\beta$ obtida a partir da função de estimação $j, \operatorname{com} j=1$ indicando EEI e $j=2$ indicando EEG;

ii. a razão entre o erro quadrático médio observado para EEI e para EEG, ou seja

$$
\mathrm{REQM}=\frac{\mathrm{EQM}_{1}}{\mathrm{EQM}_{2}}
$$

com

$$
\mathrm{EQM}_{j}=\sum_{i=1}^{1000} \frac{\left(\hat{\beta}_{i j}-0,2\right)^{2}}{1000},
$$

sendo o índice $j$ definido como no item $\mathrm{i}$.

\section{Resultados}

As Tabelas 4.1 e 4.2, trazem os índices REQM e EAM\% avaliados nas diferentes situações simuladas para a comparação de EEI com EEG. Como seria esperado, em $\rho=0$ os estimadores obtidos a partir de EEI são melhores do que os obtidos utilizando-se EEG. No entanto, conforme $n$ aumenta, a vantagem de EEI sobre EEG diminui. De um modo geral, EEG tem um comportamento melhor do que EEI para valores mais altos de $\rho$ e sua vantagem aumenta conforme $n$ e $\lambda$ aumentam. O desempenho de EEG em relação a EEI é mais fraco quando considera-se um valor maior para $t$ e menor para $n$. 
Tabela 4.1: REQM

\begin{tabular}{cc|rrr|rrr}
\hline \hline & & \multicolumn{6}{|c}{$\mathrm{t}$} \\
\cline { 3 - 8 } & & \multicolumn{3}{|c|}{5} & & \multicolumn{3}{c}{12} \\
\cline { 3 - 8 } & \multicolumn{3}{|c|}{$\rho$} & & \multicolumn{3}{c}{$\rho$} \\
\cline { 3 - 8 } $\mathrm{n}$ & $\lambda$ & 0, &, 5 &, 9 & 0, &, 5 &, 9 \\
\hline 20 & 7 & 0,71 & 1,12 & 5,45 & 0,31 & 0,52 & 2,62 \\
& 2 & 0,72 & 1,05 & 4,65 & 0,31 & 0,50 & 2,31 \\
& 1 & 0,70 & 0,89 & 0,97 & 0,33 & 0,41 & 1,38 \\
\hline 50 & 7 & 0,89 & 1,50 & 7,25 & 0,71 & 1,22 & 6,38 \\
& 2 & 0,91 & 1,38 & 5,99 & 0,72 & 1,12 & 5,60 \\
& 1 & 0,90 & 1,13 & 4,07 & 0,73 & 0,96 & 0,96 \\
\hline 100 & 7 & 0,94 & 1,48 & 7,69 & 0,88 & 1,43 & 8,00 \\
& 2 & 0,96 & 1,39 & 6,75 & 0,87 & 1,32 & 6,15 \\
& 1 & 0,96 & 1,18 & 4,45 & 0,88 & 1,10 & 4,07 \\
\hline \hline
\end{tabular}

\subsubsection{Estudo 2}

O objetivo deste estudo é comparar as estimativas obtidas a partir de EEI e EEG (ver Seção 4.2.2) com estimativas de máxima verossimilhança obtidas a partir da raiz de (4.2). Devido a limitações computacionais não foram simuladas as configurações com $n=100$ e $\lambda=1$. O fato de se considerar a matriz $\Sigma$ conhecida, faz com que as estimativas de máxima verossimilhança sejam obtidas sob condições bastantes favoráveis. Em contrapartida, cabe lembrar, que as estimativas obtidas a partir de EEI e EEG foram obtidas sob condições desfavoráveis, uma vez que não se utilizou nenhuma informação sobre a forma da estrutura de correlação dos dados.

\section{Algoritmo de estimação}

Escolheu-se o algoritmo de Newton para a obtenção das estimativas de máxima verossimilhança.

Considerando $\Sigma^{-1}=\left[\epsilon_{j k}\right], j, k=1,2, \ldots, t$, vem que os estimadores de 
Tabela 4.2: EAM $\% \times 10^{2}$

\begin{tabular}{|c|c|c|c|c|c|c|c|c|}
\hline \multirow[b]{4}{*}{$\mathrm{n}$} & \multirow[b]{4}{*}{$\lambda$} & \multirow[b]{4}{*}{$\mathrm{EE}$} & \multicolumn{6}{|c|}{$\mathrm{t}$} \\
\hline & & & \multicolumn{3}{|c|}{5} & \multicolumn{3}{|c|}{12} \\
\hline & & & \multicolumn{3}{|c|}{$\rho$} & \multicolumn{3}{|c|}{$\rho$} \\
\hline & & & 0 , &, 5 & ,9 & 0 &, 5 &, 9 \\
\hline \multirow[t]{6}{*}{20} & 7 & I & 9,0 & 8,9 & 8,8 & 5,8 & 5,8 & 5,8 \\
\hline & & $\mathrm{G}$ & 10,7 & 8,4 & 3,8 & 10,1 & 8,0 & 3,5 \\
\hline & 2 & I & 20,1 & 19,7 & 19,6 & 13,1 & 13,2 & 13,2 \\
\hline & & $\mathrm{G}$ & 23,6 & 19,4 & 9,1 & 22,5 & 18,4 & 8,5 \\
\hline & 1 & I & 35,4 & 35,5 & 36,7 & 23,2 & 23,4 & 23,5 \\
\hline & & $\mathrm{G}$ & 42,2 & 37,8 & 22,4 & 39,3 & 34,6 & 19,3 \\
\hline \multirow[t]{6}{*}{50} & 7 & I & 5,5 & 5,5 & 5,6 & 3,6 & 3,7 & 3,6 \\
\hline & & $\mathrm{G}$ & 5,8 & 4,6 & 2,1 & 4,3 & 3,3 & 1,4 \\
\hline & 2 & I & 12,0 & 12,4 & 12,5 & 7,9 & 8,2 & 8,1 \\
\hline & & $\mathrm{G}$ & 12,6 & 10,8 & 5,2 & 9,4 & 7,7 & 3,5 \\
\hline & 1 & I & 20,8 & 22,4 & 22,1 & 13,6 & 14,4 & 14,4 \\
\hline & & $\mathrm{G}$ & 21,9 & 20,9 & 10,9 & 16,0 & 14,7 & 14,7 \\
\hline \multirow[t]{6}{*}{100} & 7 & $\mathrm{I}$ & 4,0 & 4,0 & 3,9 & 2,6 & 2,6 & 2,6 \\
\hline & & G & 4,1 & 3,3 & 1,4 & 2,8 & 2,2 & 0,9 \\
\hline & 2 & I & 8,8 & 8,9 & 8,9 & 5,8 & 5,9 & 5,7 \\
\hline & & $\mathrm{G}$ & 8,9 & 7,4 & 3,5 & 6,2 & 5,2 & 2,3 \\
\hline & 1 & I & 15,0 & 15,3 & 15,6 & 10,0 & 10,3 & 10,0 \\
\hline & & $\mathrm{G}$ & 15,3 & 14,0 & 7,4 & 10,6 & 10,0 & 5,0 \\
\hline
\end{tabular}


máxima verossimilhança de $\alpha$ e $\beta$ são dados como solução de

$$
\begin{aligned}
& \hat{\alpha}=\frac{1}{n \sum_{j=1}^{t} \sum_{l=1}^{t} \epsilon_{j l}} \sum_{i=1}^{n} \sum_{j=1}^{t} \sum_{l=1}^{t} \sum_{\mathbf{k}_{i} \in \mathcal{Z}^{t}} \epsilon_{j l} \frac{e_{i \mathbf{k}}}{e_{i}}\left\{y_{i l}+2 k_{l} \pi-2 \operatorname{atan}\left(\beta x_{i l}\right)\right\} \\
&= \frac{1}{n \sum_{j=1}^{t} \sum_{l=1}^{t} \epsilon_{j l}} \sum_{i=1}^{n} \sum_{j=1}^{t} \sum_{l=1}^{t} \epsilon_{j l}\left\{y_{i l}-2 \operatorname{atan}\left(\beta x_{i l}\right)\right\} \\
&+\frac{2 \pi}{n \sum_{j=1}^{t} \sum_{l=1}^{t} \epsilon_{j l}} \sum_{i=1}^{n} \sum_{j=1}^{t} \sum_{l=1}^{t} \sum_{\mathbf{k}_{i} \in \mathcal{Z}^{t}} \epsilon_{j l} \frac{e_{i \mathbf{k}}}{e_{i}} k_{l} \mathrm{e} \\
& \sum_{i=1}^{n} \sum_{j=1}^{t} \sum_{l=1}^{t} x_{i j} h_{i j} \epsilon_{j l}\left(y_{i l}-\mu_{i l}\right)+2 \pi \sum_{i=1}^{n} \sum_{j=1}^{t} \sum_{l=1}^{t} \sum_{\mathbf{k}_{i} \in \mathcal{Z}^{t}} x_{i j} h_{i j} \epsilon_{j l} \frac{e_{i \mathbf{k}}}{e_{i}} k_{l}=0 .
\end{aligned}
$$

$\mathrm{Na}$ expressão (4.2) aparece uma série em $\mathcal{Z}$. Decidiu-se aproximar seu cálculo por uma soma de $-l$ a $l$, onde $l=5[\sigma]+1$, onde $[\cdot]$ indica o maior inteiro contido no número e $\sigma$ é o desvio-padrão da normal utilizada na geração da normal arqueada. Esse intervalo foi escolhido pois quase a totalidade da área sob a densidade da normal está contida nele.

Os valores iniciais do processo iterativo foram os mesmos do Estudo 1.

Não houve problemas com a convergência do algoritmo.

\section{Avaliação dos resultados}

A comparação das estimativas baseia-se no cálculo do erro absoluto médio relativo calculado para cada combinação de $n, t, \lambda, \rho$ e função de estimação (EEI, EEG e EEV, para a função escore).

\section{Resultados}

Nas situações com $\rho=0$, poucas diferenças, em termos do EAM, foram encontradas entre os estimadores de máxima verossimilhança e os obtidos de EEI. Isso era esperado, uma vez que, como foi dito anteriormente, a distribuição von Mises e a normal arqueada univariada são muito parecidas. Já as estimativas obtidas a partir de EEG tiveram pior comportamento nesse caso. 
Tabela 4.3: EAM

\begin{tabular}{|c|c|c|c|c|c|c|c|c|}
\hline \multirow[b]{4}{*}{$\mathrm{n}$} & \multirow[b]{4}{*}{$\lambda$} & \multirow[b]{4}{*}{$\mathrm{EE}$} & \multicolumn{6}{|c|}{$\mathrm{t}$} \\
\hline & & & \multicolumn{3}{|c|}{5} & \multicolumn{3}{|c|}{12} \\
\hline & & & \multicolumn{3}{|c|}{$\rho$} & \multicolumn{3}{|c|}{$\rho$} \\
\hline & & & 0 &, 5 & 9 & 0 &, 5 &, 9 \\
\hline \multirow[t]{6}{*}{20} & 7 & $\mathrm{~V}$ & 0,0178 & 0,0171 & 0,0065 & 0,0128 & 0,0089 & 0,0038 \\
\hline & & I & 0,0180 & 0,0177 & 0,0180 & 0,0116 & 0,0116 & 0,0116 \\
\hline & & $\mathrm{G}$ & 0,0213 & 0,0171 & 0,0076 & 0,0202 & 0,0160 & 0,0070 \\
\hline & 2 & V & 0,0380 & 0,0311 & 0,0139 & 0,0248 & 0,0193 & 0,0091 \\
\hline & & I & 0,0403 & 0,0397 & 0,0411 & 0,0262 & 0,0265 & 0,0263 \\
\hline & & $\mathrm{G}$ & 0,0472 & 0,0391 & 0,0185 & 0,0451 & 0,0368 & 0,0170 \\
\hline \multirow[t]{6}{*}{50} & 7 & $\mathrm{~V}$ & 0,0118 & 0,0099 & 0,0035 & 0,0086 & 0,0058 & 0,0025 \\
\hline & & I & 0,0109 & 0,0109 & 0,0109 & 0,0072 & 0,0073 & 0,0072 \\
\hline & & $\mathrm{G}$ & 0,0116 & 0,0091 & 0,0040 & 0,0086 & 0,0066 & 0,0028 \\
\hline & 2 & V & 0,0228 & 0,0181 & 0,0080 & 0,0160 & $0,012\rceil$ & 0,0053 \\
\hline & & I & 0,0241 & 0,0243 & 0,0246 & 0,0159 & 0,0164 & 0,0163 \\
\hline & & $\mathrm{G}$ & 0,0253 & 0,0208 & 0,0096 & 0,0187 & 0,0155 & 0,0070 \\
\hline
\end{tabular}


Conforme $\rho$ aumenta, as estimativas obtidas a partir de EEG tendem a superar as obtidas de EEI e se aproximar das de máxima verossimilhança. Isso fica mais evidente quando se toma $n=50$ e $\lambda=7$.

\subsubsection{Conclusões}

Para as situações simuladas, nota-se que há vantagens na utilização de EEG nos casos em que as correlações são altas. A medida que o tamanho da amostra aumenta, essa vantagem torna-se mais evidente.

Quando a performance de EEG foi comparada com a do método de máxima verossimilhança, nota-se que as diferenças de desempenho tendem a diminuir nas situações descritas no parágrafo anterior. Dadas as dificuldades computacionais para a obtenção dos estimadores de máxima verossimilhança (mesmo considerando-se $\Sigma$ conhecida), parece razoável sugerir a utilização de EEG nesses casos.

Cabe ressaltar que nos casos onde a variabilidade é alta, existem muitos parâmetros de perturbação e as correlações entre as medidas de um mesmo indivíduo são altas, podendo haver problemas de convergência do algoritmo de estimação, caso o tamanho amostral seja relativamente pequeno. 


\section{Capítulo 5}

\section{Aplicação}

Nesta seção um conjunto de dados reais é analisado através do ajuste de um modelo misto a dados circulares longitudinais. Esses dados foram fornecidos pelo Prof. Dr. R. Ranvaud do Institutc de Ciências Biológicas da USP, tendo sido parcialmente analisados em Ranvaud et al. (1983).

\subsection{O conjunto de dados}

Os dados correspondem à diferença entre a direção tomada por pombos-correio após 30, 60 e 90 segundos da soltura e o ângulo correspondente à sua posição no momento em que desaparecem no horizonte. Eles são parte de um estudo mais amplo realizado por vários anos na cidade de Camocim, localizada a $300 \mathrm{~km}$ de Fortaleza, no Estado do Ceará. Na época da realização do experimento, o equador magnético da Terra encontrava-se próximo a essa cidade. Um dos objetivos do estudo é investigar o mecanismo de orientação de vôo desses animais. Foram considerados dois locais de soltura (CMC-I e CMC-II); esses locais foram escolhidos devido a características de seu relevo que favorecem a observação desses animais. Para verificar o efeito da luz solar na orientação das aves, diferentes pombos foram soltos em três períodos do dia: pela manhã, próximo ao meio-dia e à tarde. Foram considerados apenas parte dos dados coletados no ano de 1982. Todos os animais eram da mesma espécie e foram transportados de seus pombais em Fortaleza até o local de soltura em gaiolas cobertas, de modo a nâo terem contato com a luz do sol até o momento da realização do experimento.

As Tabelas 5.1 e 5.2 trazem, respectivamente, a média circular amostral 
Tabela 5.1: Médias circulares amostrais relativas às diferenças entre as direções tomadas pelos pombos e o ângulo de desaparecimento

\begin{tabular}{|c|c|c|c|c|c|}
\hline \multirow[b]{2}{*}{ local } & \multirow[b]{2}{*}{ período } & \multicolumn{3}{|c|}{ tempo após soltura } & \multirow[b]{2}{*}{$\mathrm{n}$} \\
\hline & & $30 \mathrm{~s}$ & $60 \mathrm{~s}$ & $90 \mathrm{~s}$ & \\
\hline \multirow{3}{*}{ I } & manhã & 10,53 & 12,45 & 10,07 & 29 \\
\hline & meio-dia & 19,93 & 21,15 & 12,71 & 37 \\
\hline & tarde & 16,91 & 13,57 & 3,40 & 30 \\
\hline \multirow{3}{*}{ II } & manhã & $-3,46$ & $-7,22$ & $-5,01$ & 28 \\
\hline & meio-dia & $-24,12$ & $-9,53$ & $-2,32$ & 28 \\
\hline & tarde & 11,14 & 9,11 & 7,27 & 25 \\
\hline
\end{tabular}

da diferença entre as direções tomadas pelos pombos e o ângulo de desaparecimento e os comprimentos das respectivas resultantes médias e estimativas do parâmetro de concentração da von Mises, calculados, respectivamente, segundo (1.1), (1.2) e (1.19). De um modo geral, a média circular aos 90 segundos após a soltura parece estar mais próxima de zero do que a 60 segundos. O parâmetro de concentração, por sua vez, aumenta com o passar do tempo de soltura.

A Tabela 5.3 traz intervalos de $95 \%$ de confiança para as médias circulares e os parâmetros de concentração ${ }^{1}$. Uma análise grosseira desses intervalos nos leva a suspeitar que talvez não haja diferenças entre as médias circulares, uma vez que seus intervalos de confiança se interceptam. Isso será testado nas seções posteriores.

Pretende-se, nesta aplicação, verificar a existência de diferenças significativas entre as direções médias e os parâmetros de concentração, quando a soltura dos pombos é realizada nos diferentes locais e nos diferentes momentos do dia e, mais ainda, como essas medidas se comportam ao longo do tempo (30, 60 e 90 segundos após a soltura).

\footnotetext{
${ }^{1}$ ver Apêndice B
} 
Tabela 5.2: Comprimento das resultantes médias $(\bar{R})$ e estimativas dos parâmetros de concentração $(\lambda)$ relativos às diferenças entre as direções tomadas pelos pombos e o ângulo de desaparecimento

\begin{tabular}{clccccccc}
\hline & & \multicolumn{6}{c}{ tempo após soltura } \\
\cline { 3 - 5 } \cline { 5 - 8 } local & período & $30 \mathrm{~s}$ & $60 \mathrm{~s}$ & $90 \mathrm{~s}$ & & $30 \mathrm{~s}$ & $60 \mathrm{~s}$ & $90 \mathrm{~s}$ \\
\hline \multirow{2}{*}{$\mathrm{I}$} & manhã & 0,71 & 0,74 & 0,86 & & 2,06 & 2,27 & 3,89 \\
& meio-dia & 0,55 & 0,58 & 0,69 & & 1,23 & 1,45 & 1,84 \\
& tarde & 0,65 & 0,78 & 0,87 & & 1,75 & 2,68 & 4,29 \\
& & & & & & & \\
\multirow{2}{*}{ II } & manhã & 0,56 & 0,72 & 0,84 & & 1,36 & 2,09 & 3,38 \\
& meio-dia & 0,45 & 0,54 & 0,68 & & 1,15 & 1,67 & 2,83 \\
& tarde & 0,65 & 0,79 & 0,93 & 2,21 & 3,37 & 6,81 \\
\hline \hline
\end{tabular}

\subsection{Modelagem do problema}

\subsubsection{Suposições}

Seja $y_{i j k r} \in(-180 ; 180]$ a observação correspondente à diferença entre a direção do pombo $i(i=1,2, \ldots, n)$, solto na localidade $r(r=1,2)$, no horário $k,(k=1$, se de manhã, 2 se ao meio-dia ou 3 se a tarde), após $j$ segundos de soltura, $j=30,60,90$. Suponha que

a. observações correspondentes a diferentes pombos sejam independentes;

b. a estrutura de dependência entre as observações tomadas 30,60 e 90 segundos após a soltura seja a mesma para todos os pombos soltos em uma mesma localidade em um mesmo período do dia;

c. a média circular de $y_{i j k r}$ seja a mesma para todos os pombos liberados no mesmo local e período do dia $\left(\mu_{i k r}(j)=\mu_{k r}(j)\right)$ e

d. $E\left\{\cos \left(y_{i j k r}-\mu_{i k r}(j)\right)\right\}=\mathcal{A}_{1}\left(\lambda_{i k r}(j)\right), \operatorname{com} \lambda_{i k r}(j)=\lambda_{k r}(j)$, ou seja, a concentração é a mesma para todos os pombos liberados no mesmo local e período do dia;

O modo como o experimento foi conduzido faz com que a suposição (a) seja razoável. As suposições (b), (c) e (d), justificam-se pelo fato dos pombos 
Tabela 5.3: Intervalos de $95 \%$ de confiança para as médias circulares e parâmetros de concentração relativos às diferenças entre as direções tomadas pelos pombos e o ângulo de desaparecimento

\begin{tabular}{|c|c|c|c|c|}
\hline \multirow[b]{2}{*}{ local } & \multirow[b]{2}{*}{ período } & \multirow[b]{2}{*}{ tempo } & \multicolumn{2}{|c|}{ Intervalo de confiança } \\
\hline & & & média circular & parâmetro de concentração \\
\hline \multirow{9}{*}{$\mathrm{J}}$. & & 30 & $(-6,98,28,04)$ & $(1,20,2,98)$ \\
\hline & manhã & 60 & $(-3,86,28,76)$ & $(1,31,3,29)$ \\
\hline & & 90 & $(-1,41,21,55)$ & $(2,20,5,83)$ \\
\hline & & 30 & $(-3,13,42,99)$ & $(0,90,1,95)$ \\
\hline & meio-dia & 60 & $(0,58,41,72)$ & $(0,95,2,07)$ \\
\hline & & 90 & $(-3,91,29,33)$ & $(1,22,2,70)$ \\
\hline & & 30 & $(-2,69,36,51)$ & $(1,04,2,57)$ \\
\hline & tarde & 60 & $(-0,76,27,90)$ & $(1,51,3,90)$ \\
\hline & & 90 & $(-7,27,14,07)$ & $(2,38,6,38)$ \\
\hline \multirow{9}{*}{ II } & & 30 & $(-28,58,21,66)$ & $(0,85,2,07)$ \\
\hline & manhã & 60 & $(-24,79,10,35)$ & $(1,22,3,09)$ \\
\hline & & 90 & $(-17,71,7,69)$ & $(1,94,5,17)$ \\
\hline & & 30 & $(-55,11,6,87)$ & $(0,72,1,71)$ \\
\hline & meio-dia & 60 & $(-32,49,13,43)$ & $(0,82,1,99)$ \\
\hline & & 90 & $(-17,81,13,17)$ & $(1,09,2,74)$ \\
\hline & & 30 & $(-7,95,30,23)$ & $(0,98,2,58)$ \\
\hline & tarde & 60 & $(-4,79,23,01)$ & $(1,48,4,10)$ \\
\hline & & 90 & $(-1,69,16,23)$ & $(3,88,11,62)$ \\
\hline
\end{tabular}


pertencerem à mesma espécie e terem estado sujeitos às mesmas condições durante todo o experimento.

\subsubsection{Modelo}

Adotou-se os seguintes modelos (doravante denominados modelo completo) para as médias circulares e para os parâmetros de concentração

$$
\begin{aligned}
\mu_{i k r}(\text { tempo }) & =2 \operatorname{atan}\left\{\beta_{0}+\beta_{0 d} x_{1 i k r}+\beta_{0 t} x_{2 i k r}+\beta_{0 l} x_{3 i k r}\right. \\
& +\beta_{0 d l} x_{1 i k r} x_{3 i k r}+\beta_{0 t l} x_{2 i k r} x_{3 i k r} \\
& +\operatorname{tempo}\left(\beta_{1}+\beta_{1 d} x_{1 i k r}+\beta_{1 t} x_{2 i k r}+\beta_{1 l} x_{2 i k r}\right. \\
& \left.\left.\beta_{1 d l} x_{1 i k r} x_{3 i k r}+\beta_{1 t l} x_{2 i k r} x_{3 i k r}\right)\right\}, \\
\lambda_{i k r}(\text { tempo }) & =\exp \left\{\gamma_{0}+\gamma_{0 d} x_{1 i k r}+\gamma_{0 t} x_{2 i k r}+\gamma_{0 l} x_{3 i k r}\right. \\
& +\gamma_{0 d l} x_{1 i k r} x_{3 i k r}+\gamma_{0 t l} x_{2 i k r} x_{3 i k r} \\
& +\operatorname{tempo}\left(\gamma_{1}+\gamma_{1 d} x_{1 i k r}+\gamma_{1 t} x_{2 i k r}+\gamma_{1 l} x_{3 i k r}\right. \\
& \left.\left.+\gamma_{1 d l} x_{1 i k r} x_{3 i k r}+\gamma_{0 t l} x_{2 i k r} x_{3 i k r}\right)\right\},
\end{aligned}
$$

onde

$$
\begin{gathered}
\text { tempo }=\left\{\begin{array}{cc}
-1 & \text { se o tempo de soltura for } 30 \text { segundos, } \\
0 & \text { se o tempo de soltura for } 60 \text { segundos, } \\
1 & \text { se o tempo de soltura for } 90 \text { segundos; }
\end{array}\right. \\
x_{1 i k r}= \begin{cases}1 & \text { se o pombo } i \text { for solto ao meio-dia, } \\
0 & \text { em caso contrário; }\end{cases} \\
x_{2 i k r}= \begin{cases}1 & \text { se o pombo } i \text { for solto a tarde, } \\
0 & \text { em caso contrário; }\end{cases} \\
x_{3 i k r}= \begin{cases}1 & \text { se o pombo } i \text { for solto em CMC-II, } \\
0 & \text { em caso contrário; }\end{cases}
\end{gathered}
$$

onde, $\mu_{i k r}$ (tempo) e $\lambda_{i k r}$ (tempo) são, respectivamente, a média circular e o parâmetro de concentração relativos à diferença entre a direção de desaparecimento e a direção dos pombos após " $60+30 \times$ tempo" do $i$-ésimo pombo solto na localidade $r(r=1$ se CMC-I e 2 se CMC-II) no período do dia $k$ ( $k=1$ se manhã, 2 se por volta de meio-dia e 3 se a tarde); $\beta_{0}$ é a tangente da metade da média circular de pombos soltos em CMC-I, no período da manhã, após 60 s da soltura; $\beta_{0 d}$ é o incremento na tangente da metade da média circular ao se passar do período de soltura matutino para meio-dia; $\beta_{0 t}$ é o incremento na tangente da metade da média circular ao se passar do período de soltura 
matutino para tarde; $\beta_{0 l}$ é o incremento na tangente da metade da média circular ao se passar de CMC-I para CMC-II; $\beta_{0 d l}$ é o incremento na tangente da metade da média circular ao se passar do período de soltura matutino em CMC-I para meio-dia em CMC-II; $\beta_{0 t l}$ é o incremento na tangente da metade da média circular ao se passar do período de soltura matutino em CMC-I para tarde em CMC-II; $\beta_{1}$ é o incremento na tangente da metade da média circular ao se passar de 30 para 60 ou de 60 para 90 segundos da soltura, de pombos soltos em CMC-I, no período da manhã; $\beta_{1 d}$ é o incremento na tangente da metade da média circular ao se passar de 30 para 60 ou de 60 para 90 segundos da soltura, quando se passa do período de soltura matutino para meio-dia; $\beta_{1 t}$ é o incremento na tangente da metade da média circular ao se passar de 30 para 60 ou de 60 para 90 segundos da soltura, quando se passa do período de soltura matutino para tarde; $\beta_{1 l}$ é o incremento na tangente da metade da média circular ao se passar de 30 para 60 ou de 60 para 90 segundos da soltura, quando se passa do local de soltura CMC-I para CMC-II; $\beta_{1 d l}$ é o incremento na tangente da metade da média circular ao se passar de 30 para 60 ou de 60 para 90 segundos da soltura, quando se passa do período de soltura matutino para meio-dia e de CMC-I para CMC-II; $\beta_{1 t l}$ é o incremento na tangente da metade da média circular ao se passar de 30 para 60 ou de 60 para 90 segundos da soltura, quando se passa do período de soltura matutino para tarde e de CMC-I para CMC-II; os parâmetros relativos ao parâmetro de concentração têm interpretações semelhantes aos das médias que possuem o mesmo índice, substituindo-se a expressão "tangente da metade da média circular", por "logaritmo do parâmetro de concentração". Trata-se de um modelo do tipo casela de referência, onde $\beta_{0 d l}, \beta_{0 t l}, \beta_{1 d l}, \beta_{1 t l}, \gamma_{0 d l}, \gamma_{0 t l}, \gamma_{1 d l}, \gamma_{1 t l}$, representam parâmetros de interação entre local e horário de soltura; $\beta_{0 d}$, $\beta_{0 t}, \beta_{1 d}, \beta_{1 t}, \gamma_{0 d}, \gamma_{0 t}, \gamma_{1 d}, \gamma_{1 t}$, correspondem ao "efeito" de horário de soltura; $\beta_{0 l}, \beta_{1 l}, \gamma_{0 l}, \gamma_{1 l}$, ao "efeito" de local de soltura e $\beta_{0}, \beta_{1}, \gamma_{0}$ e $\gamma_{1}$ aos "efeitos" principais.

\subsection{Procedimento de estimação dos modelos}

Optamos, em um primeiro momento, pela utilização de uma função baseada em (3.25). Detalhes sobre essa função podem ser encontrados na Seção 5.6.

A matriz de covariância das funções geradoras da classe $\mathcal{L}$ foi considerada constante para cada combinação de local e período de soltura.

Há, para a função escolhida, a necessidade de estimar, para cada combi- 
nação de período e local de soltura, 12 parâmetros, correspondentes às componentes da matriz de covariâncias do seno e do cosseno das observações, centradas nas respectivas médias circulares. Um modo de diminuir o número desses parâmetros de perturbação é impor alguma estrutura à matriz de correlação correspondentc a essas funções. No entanto, observando-se essas matrizes ${ }^{2}$, quando se considera as médias dispostas na Tabela 5.1, parece não haver uma estrutura comum a todas elas. Desse modo, optou-se por utilizar uma estrutura livre, ou seja, todas as componentes da matriz foram estimadas separadamente através do estimador (3.12).

$\mathrm{Na}$ Seção 5.4.1 os resultados são comparados com os obtidos através da função de estimação baseada em (3.26), onde substitui-se a matriz $\mathbf{W}_{i}$ por $\mathbf{W}_{i}\left(\hat{\boldsymbol{\alpha}}_{n}\right)$. Não se utilizou a função de estimação baseada na ótima em $\mathcal{L}$, devido ao grande número de parâmetros de perturbação envolvidos em sua determinação (21 por combinação de local e período de soltura) em relação ao número de observações.

O procedimento de análise consiste no ajuste do modelo completo e na realização de testes de hipóteses, do tipo apresentado na Seção 3.1.5, a fim de verificar a possibilidade de ajuste de modelos reduzidos. Como critério geral para redução do modelo, tentou-se, em um primeiro momento, remover parâmetros relativos à média circular e, posteriormente, os relativos ao parâmetro de concentração. Adotou-se essa estratégia pois o parâmetro de concentração baseia-se na dispersão das observações em relação à média circular, o que torna razoável que inicialmente ajuste-se a média.

Utilizamos, como método de estimação, o algoritmo modificado de Newton apresentado na Seção 3.3.1.

O critério de parada adotado para o algoritmo de estimação foi que o máximo das diferenças entre duas estimativas consecutivas dos parâmetros e entre as respectivas componentes da função de estimação fosse inferior a $10^{-6}$.

Houve problemas de convergência para a estimação do modelo completo. Esses estavam relacionados à escolha dos valores iniciais do algoritmo de estimação. Várias opções foram utilizadas e em nenhuma delas obteve-se a convergência. Para contornar esse problema, optou-se por adotar a seguinte reparametrização:

$$
\begin{aligned}
& \mu_{k r}(\text { tempo })=2 \operatorname{atan}\left(\beta_{0 k r}+\beta_{1 k r} \text { tempo }\right), \\
& \lambda_{k r}(\text { tempo })=\exp \left(\gamma_{0 k r}+\gamma_{1 k r} \text { tempo }\right),
\end{aligned}
$$

\footnotetext{
${ }^{2}$ ver Apêndice $\mathrm{E}$
} 
Tabela 5.4: Número de iterações do algoritmo de estimação em função dos valores iniciais do processo iterativo

\begin{tabular}{rrrrrr}
\hline \hline & \multicolumn{5}{c}{$\beta^{0}$} \\
\cline { 2 - 6 }$\gamma^{0}$ & $-0,3$ & $-0,1$ & 0,0 & 0,1 & 0,3 \\
\hline$-1,0$ & $\mathrm{NC}$ & $\mathrm{NC}$ & $\mathrm{NC}$ & $\mathrm{NC}$ & $\mathrm{NC}$ \\
$-0,5$ & $\mathrm{NC}$ & $\mathrm{NC}$ & 8 & 8 & 10 \\
0,0 & 14 & 10 & 9 & 9 & 12 \\
0,5 & $\mathrm{NC}$ & 10 & 8 & 8 & 10 \\
1,0 & $\mathrm{NC}$ & $\mathrm{NC}$ & 11 & 10 & $\mathrm{NC}$ \\
\hline \hline
\end{tabular}

utilizando-se zero como valor inicial dos parâmetros relativos às médias e às concentrações. A convergência ocorreu após 9 iterações. Obtidas as estimativas, através de simples operações aritméticas envolvendo os parâmetros estimados (com quatro casas decimais), obteve-se novos valores iniciais para o modelo anterior. Utilizando-se esses valores, houve convergência após 10 iterações.

A fim de avaliar o comportamento desse algoritmo em função dos valores iniciais, o modelo (5.1) foi estimado a partir de diferentes escolhas desses valores. A Tabela 5.4 resume esses resultados. Considerou-se valores iniciais tais que $\gamma_{0 k r}=\gamma_{1 k r}=\gamma^{0}$ e $\beta_{0 k r}=\beta_{1 k r}=\beta^{0}$. Constatou-se uma grande quantidade de situações em que o algoritmo não convergiu (NC). No entanto, quando houve convergência, as estimativas obtidas sempre foram bastante próximas das obtidas anteriormente (a maior diferença entre as estimativas de um mesmo parâmetro foi da ordem de $10^{-9}$ ). Isso ilustra que dificuldades podem surgir para escolha dos valores iniciais. Tentou-se também obter os valores iniciais através da estimação de um modelo supondo-se independência entre as observações; no entanto a dificuldade de se obter valores iniciais para o algoritmo de estimação desse novo modelo foi a mesma que a do original.

\subsection{Análise dos dados}

$\mathrm{Na}$ Tabela 5.5 são apresentadas as estimativas dos parâmetros do modelo completo, $\hat{\theta}_{n}$, seus respectivos erros-padrão, ep, a razão entre essas medidas, que, sob a hipótese dos respectivos parâmetros serem iguais a zero, segue, assintoticamente, uma distribuição normal padrão e os níveis descritivos as- 
sociados aos testes de hipótese que visam verificar se os parâmetros diferem de zero. Nota-se que os erros-padrão, de um modo geral, são bastante altos.

As Tabelas 5.6 e 5.7 trazem as estimativas, segundo o modelo completo ajustado, respectivamente, das médias circulares e dos parâmetros de concentração segundo os diferentes pontos e períodos de soltura e o tempo decorrido após a soltura. Percebe-se uma certa proximidade entre os valores dessas tabelas com os das Tabelas 5.1 e 5.2, o que grosseiramente indica um bom ajuste do modelo.

Conforme mencionado, buscou-se reduzir o modelo iniciando com a eliminação dos parâmetros relativos às médias circulares, desse modo, testou-se, conjuntamente, se algum desses parâmetros, com exceção de $\beta_{0}$ diferia de zero. O valor da estatística de teste foi de 15,7486 com 11 graus de liberdade, resultando um nível descritivo de 0,1507 . Foi, em seguida, ajustado $1 \mathrm{~m}$ novo modelo com $\beta_{0}$ e os parâmetros relativos à concentração.

A Tabela 5.8 traz as estimativas dos parâmetros do primeiro modelo reduzido. Testou-se, então, se os parâmetros relativos à concentração, com exceção de $\gamma_{0}$ e $\gamma_{0 d}$, diferiam de zero. O valor observado da estatística do teste foi 50,5715, com 10 graus de liberdade e nível descritivo inferior a 0,0001 . Esse resultado indica que mais algum parâmetro deve ser mantido no modelo. Observando-se os dados iniciais, parece razoável supor que existam diferenças ao longo do tempo e, desse modo, repetiu-se o teste anterior excluindo-se $\gamma_{1}$ da hipótese: o valor da estatística de teste foi de 10,2423, com 9 graus de liberdade e nível descritivo de 0,3312 .

No modelo seguinte, manteve-se os parâmetros $\beta_{0}, \gamma_{0}, \gamma_{0 d}$ e $\gamma_{1}$. A Tabela 5.9 apresenta as estimativas desses parâmetros. Observando-se os níveis descritivos associados às hipóteses de cada parâmetro ser igual a zero, decidiu-se por não fazer novas alterações. A média circular estimada pelo modelo foi 5,07 graus. Na Tabela 5.10 são apresentados os parâmetros de concentração previstos pelo modelo.

\subsubsection{Conclusão da análise}

O modelo final apresenta certa lógica, uma vez que indica que a concentração das diferenças entre os ângulos observados após a soltura e o de desaparecimento aumenta com o passar do tempo, sendo sempre menor ao meio-dia. Pode-se especular que isso se deva ao fato de nesse momento o sol encontrar-se praticamente a pino o que impede o seu uso por parte dos pombos como orientador do caminho a ser seguido. 
Tabela 5.5: Estimativas dos parâmetros do modelo completo

\begin{tabular}{lrrrr}
\hline \hline $\boldsymbol{\theta}$ & $\hat{\boldsymbol{\theta}}_{n}$ & erro-padrão (ep) & $\hat{\boldsymbol{\theta}}_{n} / \mathrm{ep}$ & nível descritivo \\
\hline$\beta_{0}$ & 0,0965 & 0,0508 & 1,8973 & 0,0578 \\
$\beta_{0 d}$ & 0,0647 & 0,0962 & 0,6730 & 0,5010 \\
$\beta_{0 t}$ & 0,0031 & 0,0749 & 0,0413 & 0,9670 \\
$\beta_{0 l}$ & $-0,1491$ & 0,0879 & $-1,6967$ & 0,0898 \\
$\beta_{0 d l}$ & $-0,1173$ & 0,1352 & $-0,8676$ & 0,3856 \\
$\beta_{0 t l}$ & 0,1301 & 0,1123 & 1,1590 & 0,2465 \\
$\beta_{1}$ & $-0,0088$ & 0,0254 & $-0,3466$ & 0,7289 \\
$\beta_{1 d}$ & $-0,0402$ & 0,0429 & $-0,9370$ & 0,3487 \\
$\beta_{1 t}$ & $-0,0552$ & 0,0425 & $-1,2994$ & 0,1938 \\
$\beta_{1 l}$ & 0,0194 & 0,0359 & 0,5406 & 0,5887 \\
$\beta_{1 d l}$ & 0,1125 & 0,0712 & 1,5800 & 0,1141 \\
$\beta_{1 t l}$ & 0,0280 & 0,0559 & 0,5002 & 0,6169 \\
$\gamma_{0}$ & 1,1949 & 0,2451 & 4,8754 & $<0,0001$ \\
$\gamma_{0 d}$ & $-0,7824$ & 0,3369 & $-2,3224$ & 0,0202 \\
$\gamma_{0 t}$ & $-0,1854$ & 0,3204 & $-0,5786$ & 0,5629 \\
$\gamma_{0 l}$ & $-0,4338$ & 0,2968 & $-1,4614$ & 0,1439 \\
$\gamma_{0 d l}$ & 0,6236 & 0,4685 & 1,3311 & 0,1831 \\
$\gamma_{0 t l}$ & 0,7364 & 0,4498 & 1,6371 & 0,1016 \\
$\gamma_{1}$ & 0,2564 & 0,1808 & 1,4181 & 0,1562 \\
$\gamma_{1 d}$ & $-0,0430$ & 0,1952 & $-0,2203$ & 0,8257 \\
$\gamma_{1 t}$ & 0,1782 & 0,2584 & 0,6894 & 0,4906 \\
$\gamma_{1 l}$ & 0,2113 & 0,2155 & 0,9803 & 0,3270 \\
$\gamma_{1 d l}$ & 0,0018 & 0,2652 & 0,0067 & 0,9946 \\
$\gamma_{1 t l}$ & $-0,0558$ & 0,3478 & $-0,1604$ & 0,8725 \\
\hline \hline
\end{tabular}


Tabela 5.6: Médias circulares relativas às diferenças entre as direções tomadas pelos pombos e o ângulo de desaparecimento, estimadas segundo o modelo completo

\begin{tabular}{clrrr}
\hline \hline & & \multicolumn{3}{c}{ tempo após soltura } \\
\cline { 3 - 5 } local & período & $30 \mathrm{~s}$ & $60 \mathrm{~s}$ & $90 \mathrm{~s}$ \\
\hline \multirow{3}{*}{ I } & manhã & 12,02 & 11,02 & 10,02 \\
& meio-dia & 23,74 & 18,31 & 12,80 \\
& tarde & 18,57 & 11,37 & 4,07 \\
& & & & \\
\multirow{4}{*}{ II } & manhã & $-7,24$ & $-6,03$ & $-4,82$ \\
& meio-dia & $-21,32$ & $-12,02$ & $-2,56$ \\
& tarde & 11,10 & 9,21 & 7,32 \\
\hline \hline
\end{tabular}

Tabela 5.7: Parâmetros de concentração $(\lambda)$ relativos às diferenças entre as direções tomadas pelos pombos e o ângulo de desaparecirnento estimados segundo o modelo completo

\begin{tabular}{clccc}
\hline \hline & & \multicolumn{3}{c}{ tempo após soltura } \\
& & \multicolumn{3}{c}{$\lambda$} \\
\cline { 3 - 5 } local & período & $30 \mathrm{~s}$ & $60 \mathrm{~s}$ & $90 \mathrm{~s}$ \\
\hline \multirow{3}{*}{ I } & manhã & 2,56 & 3,30 & 4,27 \\
& meio-dia & 1,22 & 1,51 & 1,87 \\
& tarde & 1,78 & 2,74 & 4,24 \\
& & & & \\
& manhã & 1,34 & 2,14 & 3,42 \\
II & meio-dia & 1,19 & 1,82 & 2.80 \\
& tarde & 2,06 & 3,71 & 6,70 \\
\hline \hline
\end{tabular}


Tabela 5.8: Estimativas dos parâmetros do primeiro modelo reduzido

\begin{tabular}{lrrrr}
\hline \hline $\boldsymbol{\theta}$ & $\hat{\boldsymbol{\theta}}_{n}$ & erro-padrão (ep) & $\hat{\boldsymbol{\theta}}_{n} / \mathrm{ep}$ & nível descritivo \\
\hline$\beta_{0}$ & 0,0452 & 0,0188 & 2,4060 & 0,0161 \\
$\gamma_{0}$ & 1,2037 & $0,26 r i 5$ & 4,5335 & $<0,0001$ \\
$\gamma_{0 d}$ & $-0,8126$ & 0,3629 & $-2,2395$ & 0,0251 \\
$\gamma_{0 t}$ & $-0,2164$ & 0,3368 & $-0,6425$ & 0,5205 \\
$\gamma_{0 l}$ & $-0,4855$ & 0,3086 & $-1,5735$ & 0,1156 \\
$\gamma_{0 d l}$ & 0,5530 & 0,4759 & 1,1620 & 0,2453 \\
$\gamma_{0 t l}$ & 0,7980 & 0,4655 & 1,7143 & 0,0865 \\
$\gamma_{1}$ & 0,2420 & 0,1911 & 1,2665 & 0,2053 \\
$\gamma_{1 d}$ & $-0,0098$ & 0,2072 & $-0,0473$ & 0,9623 \\
$\gamma_{1 t}$ & 0,2095 & 0,2634 & 0,7955 & 0,4263 \\
$\gamma_{1 l}$ & 0,2050 & 0,2251 & 0,9109 & 0,3623 \\
$\gamma_{1 d l}$ & 0,0966 & 0,2921 & 0,3308 & 0,7408 \\
$\gamma_{1 t l}$ & $-0,0616$ & 0,3543 & $-0,1739$ & 0,8619 \\
\hline \hline
\end{tabular}

Tabela 5.9: Estimativas dos parâmetros do segundo modelo reduzido

\begin{tabular}{lrrrr}
\hline \hline $\boldsymbol{\theta}$ & $\hat{\boldsymbol{\theta}}_{n}$ & erro-padrão (ep) & $\hat{\boldsymbol{\theta}}_{n} / \mathrm{ep}$ & nível descritivo \\
\hline$\beta_{0}$ & 0,0335 & 0,0162 & 2,0646 & 0,0018 \\
$\gamma_{0}$ & 1,2804 & 0,1860 & 6,8833 & $<0,0001$ \\
$\gamma_{0 d}$ & $-0,2810$ & 0,2662 & $-1,0555$ & 0,0133 \\
$\gamma_{\mathbf{1}}$ & 0,5192 & 0,0674 & 7,7068 & $<0,0001$ \\
\hline \hline
\end{tabular}

Tabela 5.10: Parâmetros de concentração $(\lambda)$ relativos às diferenças entre as direções tomadas pelos pombos e o ângulo de desaparecimento estimados segundo o modelo reduzido

\begin{tabular}{cccc}
\hline \hline & \multicolumn{3}{c}{ tempo após soltura } \\
\cline { 2 - 4 } período & $30 \mathrm{~s}$ & $60 \mathrm{~s}$ & $90 \mathrm{~s}$ \\
\hline manhã / tarde & 2,02 & 2,87 & 4,09 \\
meio-dia & 1,23 & 1,75 & 2,49 \\
\hline \hline
\end{tabular}




\subsection{Comparações}

Nesta seção, compara-se o desempenho da função de estimação utilizada, doravante denominada $\Upsilon_{n}^{o}$, com a (3.29), removendo-se a linha relativa a $s_{i}$ e substituindo-se a riatriz $\mathbf{W}_{i}$ por sua estimativa, aqui denominada $\Upsilon_{n}$.

A Tabela 5.11 apresenta os parâmetros do modelo completo estimados pela raiz de $\Upsilon_{n}$. Nota-se que os parâmetros padronizados (divididos por seus erros-padrão) são, em geral, menores do que os obtidos com a função $\Upsilon_{n}^{o}$. Isso indica uma melhor performance dessa função. No entanto, em termos puramente qualitativos, os resultados descritos na Tabela 5.11 não diferem em demasia dos obtidos na Tabela 5.5. Testou-se a hipótese dos parâmetros relativos à média circular, com exceção de $\beta_{0}$, serem nulos e obteve-se uma estatística de teste de 15,1473 , com 11 graus de liberdade e nível descritivo 0,1759 , valores muito próximos aos encontrados no modelo anterior.

Seguindo-se os passos da análise descritos na Seção 5.4, ajustou-se um primeiro modelo reduzido com $\beta_{0}$ e todos os parâmetros relativos à concentração (ver Tabela 5.12). De um modos geral, as estimativas divididas por seus erros-padrão são, em sua maioria, menores do que as obtidas anteriormente. Apesar dos resultados não serem muito distintos daqueles da Tabela 5.8, duas diferenças são dignas de nota: o nível descritivo relativo à hipótese $\gamma_{d}=0$, que era 0,0251 , passa a ser 0,1096 e o relativo a $\gamma_{1}=0$ que era 0,2053 , passa a 0,0464, ou seja, caso essas fossem as únicas hipóteses de interesse, chegar-se-ia a conclusões diferentes.

Testou-se, a seguir, se os parâmetros da concentração, com exceção de $\gamma_{0}$, $\gamma_{0 d}$ e $\gamma_{1}$, diferiam de zero e obteve-se um valor da estatística do teste de 8,3228 , com 9 graus de liberdade e nível descritivo de 0,5020, valores ligeiramente inferiores aos encontrados na Seção 5.4. No entanto, se o parâmetro $\gamma_{0 d}$ fosse incluído no teste de hipótese, o valor da estatística seria de 12,3228 , com 10 graus de liberdade e nível descritivo 0,2640 , ou seja, seríamos levados a excluir esse parâmetro do modelo final.

Por fim, como o objetivo desta seção é comparar os desempenhos das duas funções de estimação, ajustou-se, através de $\Upsilon_{n}$, o mesmo modelo final obtido através de $\Upsilon_{n}^{o}$. Os resultados desse ajuste encontram-se na Tabela 5.13. Como anteriormente, os resultados obtidos, são muito próximos aos anteriores. 
Tabela 5.11: Estimativas dos parâmetros do modelo completo, segundo $\Upsilon_{n}$

\begin{tabular}{lrrrr}
\hline \hline $\boldsymbol{\theta}$ & $\hat{\boldsymbol{\theta}}_{n}$ & erro-padrão & $\hat{\boldsymbol{\theta}}_{n} /$ ep & nível descritivo \\
\hline$\beta_{0}$ & 0,0986 & 0,0552 & 1,7860 & 0,0741 \\
$\beta_{0 d}$ & 0,0617 & 0,0989 & 0,6241 & 0,5325 \\
$\beta_{0 t}$ & 0,0012 & 0,0781 & 0,0158 & 0,9874 \\
$\beta_{0 l}$ & $-0,1511$ & 0,0905 & $-1,6685$ & 0,0952 \\
$\beta_{0 d l}$ & $-0,1139$ & 0,1381 & $-0,8249$ & 0,4094 \\
$\beta_{0 t l}$ & 0,1319 & 0,1144 & 1,1527 & 0,2490 \\
$\beta_{1}$ & $-0,0102$ & 0,0282 & $-0,3628$ & 0,7168 \\
$\beta_{1 d}$ & $-0,0383$ & 0,0445 & $-0,8618$ & 0,3888 \\
$\beta_{1 t}$ & $-0,0539$ & 0,0444 & $-1,2139$ & 0,2248 \\
$\beta_{1 l}$ & 0,0208 & 0,0379 & 0,5481 & 0,5836 \\
$\beta_{1 d l}$ & 0,1102 & 0,0733 & 1,5032 & 0,1328 \\
$\beta_{1 t l}$ & 0,0267 & 0,0573 & 0,4667 & 0,6407 \\
$\gamma_{0}$ & 0,9626 & 0,2567 & 3,7507 & 0,0002 \\
$\gamma_{0 d}$ & $-0,5622$ & 0,3455 & $-1,6270$ & 0,1037 \\
$\gamma_{0 t}$ & 0,0401 & 0,3309 & 0,1213 & 0,9035 \\
$\gamma_{0 l}$ & $-0,2067$ & 0,3091 & $-0,6688$ & 0,5037 \\
$\gamma_{0 d l}$ & 0,3703 & 0,4791 & 0,7729 & 0,4396 \\
$\gamma_{0 t l}$ & 0,5124 & 0,4572 & 1,1206 & 0,2625 \\
$\gamma_{1}$ & 0,3201 & 0,1521 & 2,1045 & 0,0353 \\
$\gamma_{1 d}$ & $-0,1203$ & 0,1709 & $-0,7041$ & 0,4813 \\
$\gamma_{1 t}$ & 0,1276 & 0,2488 & 0,5130 & 0,6080 \\
$\gamma_{1 l}$ & 0,1392 & 0,2042 & 0,6817 & 0,4954 \\
$\gamma_{1 d l}$ & 0,1184 & 0,2617 & 0,4523 & 0,6511 \\
$\gamma_{1 t l}$ & $-0,0296$ & 0,3529 & $-0,0839$ & 0,9331 \\
\hline \hline
\end{tabular}


Tabela 5.12: Estimativas dos parâmetros do primeiro modelo reduzido. obtidas através de $\Upsilon_{n}$

\begin{tabular}{lrrrr}
\hline \hline $\boldsymbol{\theta}$ & $\hat{\boldsymbol{\theta}}_{n}$ & erro-padrão & $\hat{\boldsymbol{\theta}}_{n} / \mathrm{ep}$ & nível descritivo \\
\hline$\beta_{0}$ & 0,0447 & 0,0189 & 2,3576 & 0,0184 \\
$\gamma_{0}$ & 0,9462 & 0,2698 & 3,5077 & 0,0005 \\
$\gamma_{0 d}$ & $-0,5863$ & 0,3664 & $-1,6002$ & 0,1096 \\
$\gamma_{0 t}$ & 0,0323 & 0,3412 & 0,0946 & 0,9246 \\
$\gamma_{0 l}$ & $-0,2339$ & 0,3140 & $-0,7448$ & 0,4564 \\
$\gamma_{0 d l}$ & 0,3538 & 0,4850 & 0,7294 & 0,4658 \\
$\gamma_{0 t l}$ & 0,5531 & 0,4682 & 1,1815 & 0,2374 \\
$\gamma_{1}$ & 0,3147 & 0,1580 & 1,9921 & 0,0464 \\
$\gamma_{1 d}$ & $-0,0992$ & 0,1776 & $-0,5589$ & 0,5762 \\
$\gamma_{1 t}$ & 0,1510 & 0,2459 & 0,6139 & 0,5393 \\
$\gamma_{1 l}$ & 0,1192 & 0,2133 & 0,5586 & 0,5765 \\
$\gamma_{1 d l}$ & 0,1931 & 0,2840 & 0,6801 & 0,4965 \\
$\gamma_{1 t l}$ & $-0,0249$ & 0,3560 & $-0,0698$ & 0,9443 \\
\hline \hline
\end{tabular}

Tabela 5.13: Estimativas dos parâmetros do modelo final, obtidas através de $\Upsilon_{n}$

\begin{tabular}{lrrrr}
\hline \hline $\boldsymbol{\theta}$ & $\hat{\boldsymbol{\theta}}_{n}$ & erro-padrão & $\hat{\boldsymbol{\theta}}_{n} / \mathrm{ep}$ & nível descritivo \\
\hline$\beta_{0}$ & 0,0436 & 0,0190 & 2,2912 & 0,0219 \\
$\gamma_{0}$ & 0,9529 & 0,1115 & 8,5477 & $<0,0001$ \\
$\gamma_{0 d}$ & $-0,5585$ & 0,2186 & $-2,5552$ & 0,0106 \\
$\gamma_{1}$ & 0,3973 & 0,0644 & 6,1675 & $<0,0001$ \\
\hline \hline
\end{tabular}




\subsubsection{Conclusões}

Em termos da estimação pontual dos parâmetros, constatou-se poucas diferenças entre as estimativas ohtidas através de $\Upsilon_{n}^{o}$ e $\Upsilon_{n}$. Em termos da precisão dessas estimativas, a função de estimação $\Upsilon_{n}^{o}$ apresentou uma performance ligeiramente melhor que $\Upsilon_{n}$. Um fato importante é que, seguindo-se o procedimento de análise previamente proposto, o modelo final segundo $\Upsilon_{n}$ seria diferente do obtido anteriormente. Então, nesse caso, a escolha da função de estimação tem um papel importante nas conclusões finais da análise: como $\Upsilon_{n}^{o}$ apresentou uma performance ligeiramente melhor do que $\Upsilon_{n}$, em termos dos erros-padrão das estimativas, indicaríamos essa função para a estimação dos modelos.

\subsection{Detalhes técnicos}

A função de estimação baseada em (3.25) é dada, no nosso exemplo, por

$$
\begin{gathered}
\Upsilon_{n}^{o}(\boldsymbol{\theta})=\sum_{i=1}^{n} \boldsymbol{\psi}_{i}^{o} \\
\boldsymbol{\psi}_{i}^{o}=\sum_{i=1}^{n}\left[\begin{array}{c}
\mathbf{X}_{i}^{\top} \mathbf{H}_{i} \mathbf{N}_{i} \hat{\mathbf{W}}_{i}^{-1} \mathbf{u}_{i} \\
\mathbf{Z}_{i}^{\top} \mathbf{F}_{i} \mathbf{M}_{i} \hat{\mathbf{W}}_{i}^{o} \varepsilon_{i}
\end{array}\right],
\end{gathered}
$$

onde $\mathbf{X}_{i}, \mathbf{H}_{i}, \mathbf{N}_{i}, \mathbf{u}_{i}, \mathbf{Z}_{i}, \mathbf{F}_{i}$ e $\varepsilon_{i}$ são como na Seção $3.3 .2 ; \hat{\mathbf{W}}_{i}$ e $\hat{\mathbf{W}}_{i}^{o}$ são, respectivamente, as estimativas de $\operatorname{Cov}\left(\mathbf{u}_{i}\right)$ e $\operatorname{Cov}\left(\varepsilon_{i}\right)$, obtidas através de (3.12).

A matriz de covariância assintótica de $\sqrt{n}\left(\hat{\boldsymbol{\theta}}_{n}-\theta\right)$ é dada por

$$
\mathbf{J}^{-1}=\lim _{n \rightarrow \infty} n(\mathbf{S})^{-1}(\mathbf{V})(\mathbf{S})^{-\top},
$$

onde

$$
\begin{gathered}
\mathbf{S}(\theta)=\left(\begin{array}{cc}
\mathbf{S}_{11} & \mathbf{0} \\
\mathbf{0} & \mathbf{S}_{22}
\end{array}\right), \\
\mathbf{S}_{11}=\sum_{i=1}^{n} \mathbf{X}_{i}^{\top} \mathbf{H}_{i} \mathbf{N}_{i} \mathbf{W}_{i}^{-1} \mathbf{N}_{i} \mathbf{H}_{i} \mathbf{X}_{i} \\
\mathbf{S}_{22}=\sum_{i=1}^{n} \mathbf{Z}_{i}^{\top} \mathbf{F}_{i} \mathbf{M}_{i} \mathbf{W}_{i}^{o-1} \mathbf{M}_{i} \mathbf{F}_{i} \mathbf{Z}_{i},
\end{gathered}
$$




$$
\mathrm{V}(\boldsymbol{\theta})=\left\{\begin{array}{ll}
\mathrm{V}_{11} & \mathrm{~V}_{12} \\
\mathrm{~V}_{12}^{\top} & \mathrm{V}_{22}
\end{array}\right\}
$$

e

$$
\begin{gathered}
\mathbf{V}_{11}=\sum_{i=1}^{n} \mathbf{X}_{i}^{\top} \mathbf{H}_{i} \mathbf{N}_{i} \mathbf{W}_{i}^{-1} \operatorname{Cov}\left(\mathbf{u}_{i}\right) \mathbf{W}_{i}^{-1} \mathbf{N}_{i} \mathbf{H}_{i} \mathbf{X}_{i} \\
\mathbf{V}_{22}=\sum_{i=1}^{n} \mathbf{Z}_{i}^{\top} \mathbf{F}_{i} \mathbf{M}_{i} \mathbf{W}_{i}^{o-1} \operatorname{Cov}\left(\varepsilon_{i}\right) \mathbf{W}_{i}^{o}{ }^{-1} \mathbf{M}_{i} \mathbf{F}_{i} \mathbf{Z}_{i} \\
\mathbf{V}_{12}=\mathbf{X}_{i}^{\top} \mathbf{H}_{i} \mathbf{N}_{i} \mathbf{W}_{i}^{-1} E\left(\mathbf{u}_{i} \varepsilon_{i}^{\top}\right) \mathbf{W}_{i}^{o-1} \mathbf{M}_{i} \mathbf{F}_{i} \mathbf{Z}_{i}
\end{gathered}
$$

Um estimador consistente de $\mathbf{J}^{-1}$ é obtido por

$$
\hat{\mathbf{J}}^{-1}=\left\{\mathbf{S}\left(\hat{\boldsymbol{\theta}}_{n}\right)\right\}^{-1}\left(\sum_{i=1}^{n} \boldsymbol{\psi}_{i}^{o} \boldsymbol{\psi}_{i}^{o \top}\right)\left\{\mathbf{S}\left(\hat{\boldsymbol{\theta}}_{n}\right)\right\}^{-\mathrm{T}}
$$

onde $\hat{\boldsymbol{\theta}}_{n}$ é o estimador de $\boldsymbol{\theta}$ obtido a partir da raiz de $\Upsilon_{n}^{o}$. 


\section{Capítulo 6}

\section{Conclusões e futuros estudos}

Os métodos propostos no Capítulo 3 são alternativas viáveis para a estimação de alguns parâmetros de um modelo de regressão envolvendo dados com estrutura longitudinal, quando não se dispõe de uma distribuição multivariada para sua modelagem. Muitas vezes, mesmo quando a distribuição dos dados é conhecida, seu uso pode ser, por razões práticas, proibitivo. É o que constatamos, por exemplo, nos estudos de simulação ao estimar um modelo baseado na distribuição normal arqueada multivariada.

A obtenção das estimativas baseia-se em suposições, nem sempre facilmente verificáveis, sobre a estrutura de correlação de certas funções dos dados. Um problema prático relacionado a isso é a escolha de tal estrutura. O usuário da técnica deve preocupar-se com a possibilidade de existência real da estrutura suposta, pois nem sempre as funções das variáveis resposta admitirão qualquer estrutura.

Dois procedimentos podem ser adotados quando não se tem certeza sobre a forma da estrutura de correlação. O primeiro consiste em não se fazer nenhuma suposição sobre ela e estimar todas as componentes da matriz de correlação (covariâncias) independentemente das demais. O segundo consiste em se utilizar diferentes estruturas e comparar os resultados assim obtidos; se a discrepância entre esses resultados não for muito alta pode-se intuir uma certa robustez dos modelos/dados em relação à escolha da estrutura de correlação.

Deve-se ser cauteloso quando se decide utilizar a técnica proposta na Seção 3.1.4. As propriedades assintóticas dos estimadores obtidos serão válidas apenas se a estrutura de correlação proposta for correta. Isso não acontece necessariamente quando estimativas independentes de $\alpha$ são utilizadas. Nessa 
situação, mesmo sob uma estrutura errada, as condições do Teorema 6 podem estar satisfeitas.

Outra crítica cabível ao método é o fato dos parâmetros de dependência serem considerados parâmetros de perturbação. Muitas vezes, em um problema multivariado, o estudo da estrutura de currelação pode ser de interesse central, o que não acontece aqui. Nessa mesma linha, pode-se criticar o fato dos parâmetros de dependência serem simples correlações, o que nem sempre acontece em distribuições multivariadas.

Apesar dos problemas apontados, as funções de estimação apresentadas constituem um método de estimação que pode ser bastante útil desde que se conheça suas limitações.

\subsection{Futuros estudos}

\subsubsection{Modelos de séries temporais com covariáveis}

Um ponto a ser desenvolvido futuramente é a modelagem de séries temporais circulares na presença de covariáveis que podem variar com o tempo. A seguir, apresentamos um modelo adequado ao caso em que a variável resposta é um modelo de dispersão e as covariáveis são fixas. Essa hipótese de covariáveis fixas nos parece passivel de crítica, uma vez que em muitos casos elas ocorrem, ou se modificam, com o avançar da série de modo aleatório. Acreditamos que outros modelos possam ser construídos de modo a podermos supor que as covariáveis sigam alguma distribuição de probabilidade.

Seja $\left\{Y_{j}\right\}$ uma série temporal tal que $Y_{j}$ é afetada pelas observações passadas e por covariáveis fixas. Seja $\left\{\mathbf{x}_{j}\right\}$ a série de covariáveis com $\mathbf{x}_{j}=$ $\left(x_{1 j}, x_{2 j}, \ldots, x_{p j}\right)^{\top}$. Defina $\Lambda_{j}=\left\{y_{j-1}, y_{j-2}, \ldots, y_{1} ; \mathbf{x}_{j}, \mathbf{x}_{j-1}, \ldots, \mathbf{x}_{1}\right\}$, como a informação passada observada, disponível no instante $j$.

A suposição básica do modelo é $y_{j} \mid \Lambda_{j} \sim \mathcal{M D}\left(\mu_{j} ; \sigma^{2}\right)$ e tal que

$$
g\left(\mu_{j}\right)=\mathbf{x}_{j}^{\top} \boldsymbol{\beta}+\sum_{i=1}^{m} \lambda_{i} f_{i}\left(\Lambda_{j}\right)
$$

onde $\boldsymbol{\beta}=\left(\beta_{1}, \ldots, \beta_{p}\right)^{\top}$ e $\boldsymbol{\lambda}=\left(\lambda_{1}, \ldots, \lambda_{m}\right)^{\top}$ são vetores paramétricos; $g$ é uma função duplamente diferenciável e inversível e $f_{i}, i=1,2, \ldots, m$, são funções duplamente diferenciáveis. Um exemplo simples pode ser construído 
fazendo-se $f_{i}\left(\Lambda_{j}\right)=y_{j-i}$. Nesse caso, tem-se que

$$
g\left(\mu_{j}\right)=\mathbf{x}_{j}^{\top} \boldsymbol{\beta}+\sum_{i=1}^{m} \lambda_{i} y_{j-i} .
$$

Esse é um processo de natureza auto-regressiva com covariáveis. Para simplificar a notação, denomine $\eta_{j}=\mathbf{x}_{j}^{\top} \boldsymbol{\beta}, \nu_{j}=\sum_{i=1}^{m} \lambda_{i} f_{i}\left(\Lambda_{j}\right), h\left(\eta_{j}\right)$ a inversa de $g\left(\mu_{j}\right)$ and $\theta=\left(\boldsymbol{\beta}^{\top}, \lambda^{\top}\right)^{\top}$.

Para o desenvolvimento da teoria, $y_{1}, y_{2}, \ldots, y_{m}$, serão considerados fixos.

Sob este modelo e com a suposição de que $f_{i}, i=1,2, \ldots, m$, são independentes de $\beta$, a função densidade de probabilidades de $Y_{j} \mid \Lambda_{j}$ é dada por

$$
p_{Y_{j} \mid \Lambda_{j}}\left(y_{j}\right)=a\left(y_{j} ; \sigma^{2}\right) \exp \left\{-\frac{1}{2 \sigma^{2}} d\left(y_{j} ; \mu_{j}\right)\right\},
$$

onde $a$ e $d$ são funções convenientes. Após $n$ realizações consecutivas da série temporal, a função de log-verossimilhança de $\vartheta$ é dada por

$$
L(\boldsymbol{\theta})=\sum_{j=m+1}^{n} \log a\left(y_{j} ; \sigma^{2}\right)-\frac{1}{2 \sigma^{2}} \sum_{j=m+1}^{n} d\left(y_{j} ; \mu_{j}\right) .
$$

Suas derivadas com respeito a $\beta_{k}, \boldsymbol{\beta}, \lambda_{i}$ e $\boldsymbol{\lambda}$ são dadas por

$$
\frac{\partial L}{\partial \beta_{k}}(\theta)=-\frac{1}{2 \sigma^{2}} \sum_{j=m+1}^{n} x_{j k} \dot{h}_{j} u_{j},
$$

onde $u_{j}=\frac{\partial d}{\partial \mu_{j}}\left(y_{j} ; \mu_{j}\right)$ e $\dot{h}_{j}=\left.\frac{\partial h}{\partial x}(x)\right|_{x=\eta_{j}+\nu_{j}}$;

$$
\frac{\partial L}{\partial \boldsymbol{\beta}}(\boldsymbol{\theta})=-\frac{1}{2 \sigma^{2}} \mathbf{X}^{\top} \mathbf{H u},
$$

onde $\mathbf{H}=\operatorname{diag}\left\{\dot{h}_{m+1}, \ldots, \dot{h}_{n}\right\}, \mathbf{u}=\left(u_{m+1}, \ldots, u_{n}\right)^{\top}$;

$$
\frac{\partial L}{\partial \lambda_{i}}(\theta)=-\frac{1}{2 \sigma^{2}} \sum_{j=m+1}^{n} f_{i}\left(\Lambda_{j}\right) \dot{h}_{j} u_{j}
$$

onde

$$
\frac{\partial L}{\partial \boldsymbol{\lambda}}(\boldsymbol{\theta})=-\frac{1}{2 \sigma^{2}} \mathbf{F}^{\top} \dot{\mathbf{H}} \mathbf{u}
$$


com

$$
\mathbf{F}=\left\{\begin{array}{cccc}
f_{1}\left(\Lambda_{m+1}\right) & f_{2}\left(\Lambda_{m+1}\right) & \ldots & f_{m}\left(\Lambda_{m+1}\right) \\
f_{1}\left(\Lambda_{m+2}\right) & f_{2}\left(\Lambda_{m+2}\right) & \ldots & f_{m}\left(\Lambda_{m+2}\right) \\
\vdots & \vdots & \ldots & \vdots \\
f_{1}\left(\Lambda_{n}\right) & f_{2}\left(\Lambda_{n}\right) & \ldots & f f_{m}\left(\Lambda_{n}\right)
\end{array}\right\}
$$

Em resumo, a função escore $\theta$ é dada por

$$
\frac{\partial L}{\partial \boldsymbol{\theta}}(\boldsymbol{\theta})=-\frac{1}{2 \sigma^{2}}\left(\begin{array}{l}
\mathbf{X}^{\top} \\
\mathbf{F}^{\top}
\end{array}\right) \mathbf{H u} .
$$

Sob condições gerais de regularidade pode-se utilizar o procedimento de Newton para estimar $\boldsymbol{\beta}$, dadas $n$ realizações do processo. A segunda derivada da log-verossimilhança é dada por

$$
\frac{\partial^{2} L}{\partial \beta_{k} \partial \beta_{l}}(\boldsymbol{\theta})=-\frac{1}{2 \sigma^{2}} \sum_{j=m+1}^{n} x_{k j}\left(\ddot{h}_{j} \mathbf{u}_{j}+\dot{h}_{j}^{2} \dot{u}_{j}\right) x_{l j},
$$

onde $\ddot{h}_{j}=\frac{\partial \dot{h}_{j}}{\partial \eta_{j}} ;$ consequentemente

$$
\frac{\partial^{2} L}{\partial \boldsymbol{\beta} \partial \boldsymbol{\beta}^{\top}}=-\frac{1}{2 \sigma^{2}}\left(\mathbf{X}^{\top} \mathbf{E X}\right)
$$

onde $\mathbf{E}=\dot{\mathbf{H}} \Delta(\mathbf{u})+\mathbf{H} \Delta(\dot{\mathbf{u}}) \mathbf{H}$,

$$
\begin{gathered}
\frac{\partial^{2} L}{\partial \beta_{k} \partial \lambda_{r}}=-\frac{1}{2 \sigma^{2}} \sum_{j=m+1}^{n} x_{k j}\left(\ddot{h}_{j} u_{j}+\dot{h}_{j} \dot{u}_{j} \dot{h}_{j}\right) f_{r}\left(\Lambda_{j}\right) \\
\frac{\partial^{2} L}{\partial \beta \partial \boldsymbol{\lambda}^{\top}}=-\frac{1}{2 \sigma^{2}} \mathbf{X}^{\top} \mathbf{E F} \\
\frac{\partial^{2} L}{\partial \lambda_{r} \partial \lambda_{s}}=-\frac{1}{2 \sigma^{2}} \sum_{j=m+1}^{n} f_{r}(\Lambda)\left(\ddot{h}_{j} u_{j}+\dot{h}_{j} \dot{u}_{j} \dot{h}_{j}\right) f_{s}(\Lambda) \\
\frac{\partial^{2} L}{\partial \boldsymbol{\lambda} \partial \boldsymbol{\lambda}^{\top}}=-\frac{1}{2 \sigma^{2}} \mathbf{F}^{\top} \mathbf{E F} .
\end{gathered}
$$

Portanto,

$$
\mathbf{K}(\theta)=\frac{\partial L}{\partial \theta \partial \theta^{\top}}=-\frac{1}{2 \sigma^{2}}\left(\begin{array}{ll}
\mathbf{X}^{\top} \mathbf{E X} & \mathbf{X}^{\top} \mathbf{E F} \\
\mathbf{F}^{\top} \mathbf{E X} & \mathbf{F}^{\top} \mathbf{E F}
\end{array}\right) .
$$


O passo $(k+1)$ do algoritmo de Newton para a estimação de $\theta$ é dado por

$$
\theta^{(k+1)}=\theta^{(k)}-\mathbf{K}^{-1} \frac{\partial L}{\partial \theta}\left(\theta^{(k)}\right) .
$$

Pode-se utilizar a matriz de informação de Fisher para determinar a matriz de covariância assintótica do estimador $\hat{\boldsymbol{\theta}}$; ela é dada por $\operatorname{Cov}_{a}=E\{-\mathbf{K}(\theta)\}$.

Diferentes modelos podem ser obtidos ao se incorporar $\boldsymbol{\beta}$ nas funções $f_{i}$, como por exemplo,

$$
f_{i}\left(\Lambda_{j}\right)=\lambda_{i}\left(y_{j-i}-\mathbf{x}_{j-i}^{\top} \boldsymbol{\beta}\right)
$$

Nesses casos tem-se

$$
\frac{\partial L}{\partial \beta_{k}}(\boldsymbol{\theta})=-\frac{1}{2 \sigma^{2}} \sum_{j=m+1}^{n} u_{j} \dot{h}_{j}\left(x_{k j}+\sum_{i=1}^{m} \dot{f}_{i k}\left(\Lambda_{j}\right)\right),
$$

onde $\dot{f}_{i j}=\frac{\partial f_{i}}{\partial \beta_{j}}$. Logo,

$$
\frac{\partial L}{\partial \boldsymbol{\theta}}(\boldsymbol{\theta})=-\frac{1}{2 \sigma^{2}}\left(\begin{array}{c}
\mathbf{X}^{\top}+\mathbf{F}_{2}^{\top} \\
\mathbf{F}^{\top}
\end{array}\right) \mathbf{H u}
$$

onde

$$
\mathbf{F}_{2}^{\top}=\left(\begin{array}{c}
\sum_{i=1}^{m} \dot{f}_{i 1} \\
\sum_{i=1}^{m} \dot{f}_{i 2} \\
\vdots \\
\sum_{i=1}^{m} \dot{f}_{i p}
\end{array}\right) \otimes \mathbf{1}_{n-m}^{\top}
$$

$\mathrm{O}$ algoritmo de Newton para a estimação de $\theta$ é dado por

$$
\theta^{(k+1)}=\theta^{(k)}-\mathbf{K}_{2}^{-1} \frac{\partial L}{\partial \boldsymbol{\theta}}\left(\boldsymbol{\theta}^{(k)}\right)
$$

onde

$$
\mathbf{K}_{2}=-\frac{1}{2 \sigma^{2}}\left\{\begin{array}{cc}
\left(\mathbf{X}^{\top}+\mathbf{F}_{2}^{\top}\right) \mathbf{E}\left(\mathbf{X}+\mathbf{F}_{2}\right) & \left(\mathbf{X}^{\top}+\mathbf{F}_{2}^{\top}\right) \mathbf{E F}_{2} \\
\mathbf{F}_{2}^{\top} \mathbf{E}\left(\mathbf{X}+\mathbf{F}_{2}\right) & \mathbf{F}_{2}^{\top} \mathbf{E F}_{2}
\end{array}\right\}
$$

\subsection{Geração de distribuições circulares mul- tivariadas}

Outro possível campo de estudo na área de dados circulares é a criação de distribuições multivariadas para a modelagem não só de dados longitudinais, como também de vetores de variáveis circulares. A distribuição normal 
arqueada multivariada apresentada nesta tese é uma tentativa de obter tal estrutura; no entanto vimos as dificuldades inerentes à estimação de seus parâmetros.

Joe (1996) ressalta as características desejáveis em uma distribuição multivarida e dentre elas destacam-se:

i. interpretabilidade - os parâmetros da distribuição devem ser facilmente interpretáveis (exemplo: na distribuição normal multivariada há parâmetros de posição, variabilidade e dependência);

ii. suas distribuições marginais e conjuntas de menor ordem devem pertencer à mesma família de distribuições (exemplo: a distribuição normal multivariada possui marginais normais e distribuições conjuntas de menor ordem normais multivariadas);

iii. a distribuição deve ser abrangente o suficiente para contemplar a modelagem de diferentes estruturas de dependência (exemplo: a distribuição normal multivariada permite a modelagem de diversas estruturas de correlação);

iv. sua função distribuição acumulada e função densidade (ou de probabilidades, no caso discreto) devem possuir forma fechada, ou pelo menos que essas funções possam ser avaliadas numericamente com facilidade (exemplo: a normal multivariada tem densidade fechada, mas não tem função distribuição fechada);

v. os parâmetros devem ser facilmente estimáveis e preferencialmente devem depender apenas das distribuições marginais ou de conjuntas de menor ordem. Joe e Xu (1996) e Xu (1996) desenvolveram um método que facilita a estimação desse tipo de parâmetro. (Por exemplo, na distribuição normal multivariada quaisquer componente do vetor de médias e da diagonal da matriz de covariâncias podem ser definidas através das distribuições marginais; entretanto, as covariâncias, por sua vez, podem ser definidas em termos das distribuições bivariadas).

De um modo geral é difícil encontrar distribuições multivariadas que satisfaçam a todas essas propriedades.

Um modo relativamente simples de obter-se um modelo multivariado com distribuições marginais pré-estabelecidas é o baseado no uso de cópulas (ver 
Joe, 1993 e Joe, 1996, por exemplo). Uma cópula $C\left(u_{1}, \ldots, u_{t}\right)$ é uma função distribuição multivariada com marginais uniformes no intervalo $(0,1)$. Uma técnica simples para construção de cópulas consiste em considerar um vetor aleatório absolutamente contínuo, $\mathrm{z}^{\top}=\left(z_{1}, \ldots, z_{t}\right)$, com função distribuição $G, \operatorname{com} z_{i}$ tendo função distribuição $G_{i}, i=1,2, \ldots, t$. Demonstra-se facilmente $^{1}$ que

$$
C(\mathbf{u})=G\left\{G_{1}^{-1}\left(u_{1}\right), G_{2}^{-1}\left(u_{2}\right), \ldots, G_{t}^{-1}\left(u_{t}\right)\right\},
$$

onde $0<u_{i}<1, i=1,2, \ldots, t$, é uma cópula.

Uma família de cópulas bastante conhecida é a gerada por uma distribuição normal multivariada. Seja $\mathbf{z} \sim \mathcal{N}_{t}(\boldsymbol{\mu}, \boldsymbol{\Sigma})$ e considere sua distribuição acumulada representada por $\Phi$, sendo $\phi_{i}$ a função distribuição de sua $i$-ésima marginal. A mencionada cópula é dada por

$$
\begin{aligned}
C(\mathbf{u}) & =\Phi\left\{\phi_{1}^{-1}\left(u_{1}\right), \phi_{2}^{-1}\left(u_{2}\right), \ldots, \phi_{t}^{-1}\left(u_{t}\right)\right\} \\
& =\int_{-\infty}^{\phi_{1}^{-1}\left(u_{1}\right)} \int_{-\infty}^{\phi_{2}^{-1}\left(u_{2}\right)} \ldots \int_{-\infty}^{\phi_{t}^{-1}\left(u_{t}\right)} \frac{1}{(2 \pi)^{t / 2}|\Sigma|^{1 / 2}} \\
& \exp \left\{-\frac{1}{2}(\mathbf{z}-\boldsymbol{\mu})^{\top} \Sigma^{-1}(\mathbf{z}-\boldsymbol{\mu})\right\} d z_{t} \ldots d z_{2} d z_{1} .
\end{aligned}
$$

É fácil constatar que essa cópula viola a propriedade iv descrita acima

A partir de uma cópula, pode-se gerar uma distribuição multivariada com marginais previamente definidas. Para gerar a função distribuição de um vetor aleatório absolutamente contínuo, y, de tal sorte que a distribuição marginal de sua $i$-ésima componente seja $F_{i}$, basta fazer ${ }^{2}$

$$
F(\mathbf{y})=C\left\{F_{1}\left(y_{1}\right), F_{2}\left(y_{2}\right), \ldots, F_{t}\left(y_{t}\right)\right\} .
$$

Essa técnica pode ser utilizada, por exemplo, para gerar uma distribuição circular $t$-variada com marginais von Mises. Seja

$$
F_{i}\left(a_{i}\right)=\int_{0}^{a_{i}} \frac{1}{2 \pi I_{o}\left(\lambda_{j}\right)} \exp \left\{\lambda_{i} \cos \left(x_{i}-\nu_{i}\right)\right\} d x_{i}, \quad i=1,2, \ldots, t
$$

com $0 \leq a_{i}<2 \pi$ e considere a cópula (6.8) gerada a partir de distribuições marginais normais padrão. Desse modo

$$
F(\mathrm{y})=\Phi\left[\phi_{1}^{-1}\left\{F_{1}\left(y_{1}\right)\right\}, \phi_{2}^{-1}\left\{F_{2}\left(y_{2}\right)\right\}, \ldots, \phi_{t}^{-1}\left\{F_{t}\left(y_{t}\right)\right\}\right] .
$$

\footnotetext{
${ }^{1}$ ver Apêndice $\mathrm{F}$

${ }^{2}$ ver demonstração no Apêndice $\mathrm{F}$
} 
$F$ viola várias das propriedades desejáveis para uma distribuiçào multivariada e a estimação de seus parâmetros a partir de uma amostra aleatória pode ser bastante trabalhosa ${ }^{3}$. As eventuais propriedades desses estimadores poderiam ser estudadas através de simulações e comparadas com os estimadores obtidos a partir da normal arqueada multivariada e das funções de estimação propostas. Outras famílias de cópulas poderiam ser utilizadas na geração de modelos circulares multivariados. Cabe ressaltar que há outros métodos de geração de distribuições multivariadas que poderiam ser empregados com essa finalidade (ver Joe, 1996, por exemplo).

\subsubsection{Estimação da matriz de covariâncias dos parâ- metros obtidos a partir das funções de estimação tipo "EEG"}

O estimador sanduíche da matriz de covariâncias dos estimadores obtidos no Capítulo 3 foi estudado no caso de marginais na família dos modelos exponenciais de dispersão. Esses estudos concluiram que o estimador obtido, apesar de consistente, é pouco eficiente. Pode-se desenvolver outro estimador obtido através de procedimentos do tipo "Jacknife/Bootstrap". Estudos de simulação podem avaliar supostas vantagens desse estimador em relação ao sanduíche.

\footnotetext{
${ }^{3}$ Joe e Xu (1996) propõem um método alternativo que facilita a estimação dos parâmetros nessas condições
} 


\section{Referências Bibliográficas}

[1] Abramowitz, M. e Stegun, I.A. (1970). Handbook of Mathematical Functions. New York, Dover Pub. 1046p.

[2] Accardi, L., Cabrera, J. e Watson, G.S. (1987). Some stationary Markov process in discrete time for unit vectors. Metron, 45, 115-133.

[3] Albert, P.S. E McShane, L.M. (1995). A generalized estimating equations approach for spatially correlated binary data: applications to the analysis of neuroimage data. Biometrics 50,627-38.

[4] Barndorff-Nielsen, O.E. E Jørgensen, B. (1991). Some parametric models on the simplex. Journal of Multivariate Analysis 39, 106-16.

[5] Batschelet, E. (1981). Circular Statistics in Biology. London, Academic Press.

[6] Bernoulli, D. (1734). Récherches Physiques et Astronomiques, Sur le Problème Proposé Pour la Seconde Fois Par L'Académie Royale des Sciences des Paris. Récuiel des Pièces Qui Ont Remportéle prix de L'Académie Royale des Sciences, Tome III, 95-134.

[7] Breckling, J. (1989). Analysis of Directional Time Series: Application to Wind Speed and Direction. Berlin, Springer-Verlag. (Lecture Notes in Statistics, 61).

[8] Brockwell, P.J. E Davis, R.A. (1991). Time Series: Theory and Method. New York, Springer-Verlag. (Springer Series in Statistics).

[9] CArr, G.J. E Chi, E.M. (1992). Analysis of variance for repeated measures data: a generalized estimation equation approach. Statistics in Medicine 11, 1033-40. 
[10] Chambers, J. (1977). Computational Methods for Data Analysis. New York, John Wiley \& Sons. (Wiley Series in Probability and Mathematical Statistics)

[11] Chandrasekar, B. E Kale, B.K. (1984). Unbiased statistical estimation functions in presence of nuisance parameter. Journai of Statistical Planning and Inference 9, 45-54.

[12] Collett, D. E Lewis, T. (1981). Discriminating between the von Mises and Wrapped Normal distributions. Australian Journal of Statistics 23, 73-79.

[13] Cologne, J.B., Cartes, R.L., Fujita, S. E Ban, S. (1993). Applications of generalized estimating equations to a study of in vitro radiation sensitivity. Biometrics 49, 927-34.

[14] Crowder, M. (1986). On consistency and inconsistency of estimating equations. Econometric Theory 2, 205-30.

[15] Crowder, M. (1987). On linear and quadratic estimating function. Biometrika 74, 591-7.

[16] Diggle, P.J., Liang, K.-Y. E Zeger, S.L. (1994). Analysis of Longitudinal Data. Oxford, Clarendon Press. (Oxford statistical science series, 13).

[17] FirTh, D. (1992). Discussão sobre o artigo Multivariate regression analysis for categorical data de Liang, K.-Y., Zeger, S.L. e Qaquish, B..Journal of Royal Statistical Society B, 54. 24-26.

[18] Fisher, N.I. (1993). Statistical Analysis of Circular Data. Cambridge, University Press.

[19] Fisher, N.I. E LeE, A.J. (1983). A correlation coefficient for circular data. Biometrika 70, 327-32.

[20] Fisher, N.I. E LEE, A.J. (1992). Regression models for an angular response. Biometrics, 48, 665-77.

[21] Fisher, N.I. E LeE, A.J. (1994). Time series analysis of circular data. Journal of the Royal Statistical Society, 56, 327-39. 
[22] Fitzmaurice, G.M., Laird, N.M. e Rotnitzky, A.G. (1993). Regression models for discrete longitudinal responses. Statistical Science, 8, 284-309. (with discussion).

[23] Godambe, V.P. (1960). An optimum property of regular maximum likelihood estimation. Annals of Mathematical Statistics, 31, 1208-11.

[24] Godambe, V.P. (1991). Estimating Functions. oxford, Oxford University Press. (Oxford Statistical Science series - 7).

[25] Godambe, V.P. E Kale, B.K. (1991). Estimating function: an overview. Em Godambe, V.P., ed., Estimating Functions. Oxford, Oxford University Press, 3-20.

[26] Gould, A.L. (1969). A regression technique for angular variates and related regression models . Biometrics, 25, 683-700.

[27] Graybill, F.A. (1969). Introduction to Matrices with Applications in Statistics. Belmont, Wadsworth Pub. Comp.

[28] Huber, P.J. (1967). The behaviour of maximum likelihood estimates under nonstandard conditions. Em Proceedings of the Fifth Berkeley Symposium. Berkeley, University of California Press, 221-33.

[29] Huber, P.J. (1981). Robust Statistics. New York, John Wiley \& Sons. (Wiley Series in Probability and Mathematical Statistics).

[30] INAGAKI, N. (1973). Asymptotic relations between the likelihood estimating function and the maximum likelihood estimator. Annals of the Institute of Statistical Mathematics, 25, 1-26.

[31] Joe, H. (1993). Parametric family of multivariate distributions with given margins. Journal of Multivariate Analysis. 46, 262-82.

[32] Joe, H. (1996). Multivariate Models and Dependence Concepts. Lecture Notes, Vancouver, UBC.

[33] JoE, H. E XU, J.J. (1996). The estimation method of inference functions for margins for multivariate models. Technical Report \# 166, Vancouver, UBC. 
[34] Johnson, R.A. E Wehrly, T.E. (1978). Some angular-linear distributions and related regression models. JASA, 73, 602-6.

[35] JøRGensen, B. (1987). Exponential dispersion models (with discussion). Journal of the Roya! Statistical Society, 49, 127-169.

[36] Jørgensen, B. (1997a). Proper dispersion models. Brazilian Journal of Probability and Statistics (a aparecer).

[37] Jørgensen, B. (1997b). The Theory of Dispersion Models. London: Chapman \& Hall (a aparecer).

[38] Jørgensen, B. E Labouriau, R.S. (1994). Exponential Families and Theoretical Inference. Lecture Notes, Department of Statistics, University of British Columbia.

[39] Jørgensen, B., Lindbye-Christensen, S., Song, X-K., Sun, L. (1995). State space models for multivariate longitudinal data of mixed types. Technical Report \# 150, UBC.

[40] Kenward, M.G., Lesaffre, E. E Molenberghs, G. (1994). An application of maximum likelihood and generalized estimating equations to the analysis of ordinal data from a longitudinal study with cases missing at random. Biometrics, 50, 945-53.

[41] LeCAm, L. (1956). On the asymptotic theory of estimation and testing hypothesis. Em Proceedings of the Third Berkeley Symposium on Mathematical Statistics and Probability, vol.1. Berkeley, University of California Press. 129-56.

[42] Lee, A.J., Scott, A.J. E Soo, S.L. (1993). Comparing Liang-Zeger estimates with maximum likelihood in bivariate logistic regression. Journal of Statistical Computing Simulation, 44, 133-48.

[43] LI, W.K. (1995). Time series models based on generalized linear models: some further results. Biometrics 50, 506-11.

[44] Liang, K.-Y. E Hanfelt, J. (1994). On the use of quasi-likelihood method in teratological experiments. Biometrics, 50, 872-80. 
[45] LiAnG, K.-Y. E ZEgER, S.L. (1995). Inference based on estimating functions in the presence of nuisance parameters. Statistical Science 10, 158-99 (with discussion).

[46] Liang, K.-Y. E ZEger, S.L. (1986). Longitudinal aralysis using generalized linear models. Biometrika 73, 13-22.

[47] Liang, K.-Y., Zeger, S.L. E QAqISH, B. (1992). Multivariate regression analysis for categorical data. Journal of the Royal Statistical Society 54, 3-40. (with discussion).

[48] Lipsitz, S.R., Fitzmaurice, G.M., Orav, R.J. E LAird, N.M. (1994). Performance of generalized estimating equations in practical situations. Biometrics 50, 270-8.

[49] Mardia, K.V. (1972). Statistics of Directional Data. London, Academic Press.

[50] McCullagh, P. e Nelder, J.A. (1989). Generalized Linear Models. 2ed. London, Chapman \& Hall.

[51] MCLeish, D.L. E Small, C.G. (1988). The Theory and Applications of Statistical Inference Functions. New York, Springer-Verlag. (Lecture Notes in Statistics, 44).

[52] Miller, M.E., Davis, C.S. E LANdis, J.R. (1993). The analysis of longitudinal polytomous data: generalized estimating equations and connections with weighted least squares. Biometrics 49, 1033-44.

[53] Nash, J.C. (1990). Compact Numerical Methods for Computers: Linear Algebra and Function Minimization, 2ed. New York, Hilger.

[54] Pepe, M.S. E Anderson, G.L. (1994). A cautionary note on inference for marginal regression models with longitudinal data and general correlated response data. Communications in Statistics B, 23. 939-51.

[55] Prentice, R.L. (1988). Correlated binary regression with covariates specific to each binary observation. Biometrics, 44, 1033-48.

[56] Prentice, R.L. E Zhao, L.P. (1991). Estimating equation for parameters in means and covariances of multivariate discrete and continuous responses. Biometrics, 47, 825-39. 
[57] Ranvaud, R., Schmidt-Koenig, K., Kiepenheuer, J. e GaspaRoTTO, O.C. (1983). Initial orientation of homing pigeons at the magnetic equator with and without sun compass. Behavioral Ecology and Sociobiology, 14, 77-79.

[58] Rivest, L-P. (1989). Spherical regression for concentrated Fisher-von Mises distributions. The Annals of Statistics, 17, 307-17.

[59] Rotnitzky, A. E Jewell, N.P. (1990). Hypothesis testing of regression parameters in semiparametric generalizer linear models for cluster correlated data. Biometrika, 77, 485-97.

[60] Sen, P.K. E Singer, J.M. (1993). Large Sample Methods in Statistics: an Introduction with Applications. New York, Chapman \& Hall, Inc.

[61] Sobol, I.M. (1975). The Monte Carlo Method. Moscow, Mir Pub. (Little mathematics library).

[62] Stephens, M.A. (1963). Random walk on a circle. Biometrika, 50, 385-90.

[63] Upton, G.J.G. E Fingleton, B. (1989). Spatial Data Analysis by Example. v.2. Chichester, John Wiley \& Sons. (Wiley Series in Probability and Mathematical Statistics).

[64] Xu, J.J. (1996). Statistical Modelling and Inference for Multivariate and Longitudinal Discrete Response Data. Ph.D. thesis, Department of Statistics, University of British Columbia.

[65] Watson, G.S. (1982). Distributions on the circle and sphere. Em Gani, J. e Hannan, E.J., eds., Essays in Statistical Science. Sheffield, Applied Probability Trust. 265- 80. (Journal of Applied Probability, special volume 19A).

[66] Wedderburn, R.W.M. (1974). Quasi-likelihood function, generalized linear models, and the Gauss-Newton method. Biometrika,61, 439-47.

[67] Whittemore, A.S. E Gong, G. (1994). Segregation analysis of casecontrol data using generalized estimating equations. Biometrics 50(4), 1073-87. 
[68] Zeger, S.L. E LiAng, K-Y (1986). Longitudinal data analysis for discrete and continuous outcomes. Biometrics, 42, 121-30.

[59] Zeger, S.L., Liang, K-Y e Albert, P.S. (1988). Models for longitudinal data: a generalized estimating equation approach. Biometrics, 44, 1049-60.

[70] Zeger, S.L., Liang, K-Y E Self, S.G. (1985). The analysis of binary longitudinal data with time-independent covariates. Biometrika, 72(1), $31-8$.

[71] Zeger, S.L. E QAQISH, B. (1988). Markov regression models for time series: a quasi-likelihood approach.Biometrics, 44, 1019-31. 


\section{Apêndice A}

\section{Funções de Bessel}

Neste apêndice serão apresentados alguns resultados relativos às funções $I_{p} \mathrm{e}$ $\mathcal{A}_{p}$ de utilidade para o estudo de propriedades da distribuição von Mises. Os resultados foram extraídos de Abramowitz e Stegun (1970), Mardia (1972) e Fisher (1993).

\section{A.1 Derivadas de $I_{0}, I_{1}$ e $\mathcal{A}_{1}$}

Inicialmente são apresentadas as derivadas de $I_{0}$ e $I_{1}$ avaliadas em um ponto $x>0$.

$$
\begin{gathered}
\frac{\mathrm{d} I_{0}}{\mathrm{~d} x}(x)=I_{1}(x), \\
\frac{\mathrm{d} I_{1}}{\mathrm{~d} x}(x)=I_{0}(x)-\frac{I_{1}(x)}{x}=\frac{1}{2}\left\{I_{0}(x)+I_{2}(x)\right\}
\end{gathered}
$$

$\mathrm{e}$

$$
\frac{\mathrm{d} x I_{1}}{\mathrm{dx}}(x)=x I_{0}(x) \text {. }
$$

São apresentadas a seguir as fórmulas das duas primeiras derivadas de $\mathcal{A}_{1}$, avaliadas no ponto $x>0$.

$\mathrm{e}$

$$
\frac{\mathrm{d} \mathcal{A}_{1}}{\mathrm{~d} x}(x)=1-\frac{\mathcal{A}_{1}(x)}{x}-\mathcal{A}_{1}^{2}(x)
$$

$$
\frac{\mathrm{d}^{2} \mathcal{A}_{1}}{\mathrm{~d} x^{2}}(x)=\frac{\mathcal{A}_{1}(x)}{x^{2}}-\frac{\mathrm{d} \mathcal{A}_{1}}{\mathrm{~d} x}(x)\left\{2 \mathcal{A}_{1}(x)+\frac{1}{x}\right\} .
$$




\section{A.2 Cálculo das funções}

Nessa seção são apresentados resultados úteis para a estimação de parâmetros de uma distribuição von Mises.

\section{A.2.1 Fórmulas de recorrência}

Para $x>0$ e $p=2,3, \ldots$, tem-se

$$
I_{p}(x)=I_{p-2}(x)-\frac{2(p-1)}{x} I_{p-1}(x)
$$

$\mathrm{e}$

$$
\mathcal{A}_{p}(x)=\mathcal{A}_{p-2}(x)-\frac{2(p-1)}{x} \mathcal{A}_{p-1}(x)
$$

\section{A.2.2 Avaliação de $I_{0}$ e $I_{1}$}

Definindo $t=x / 3,75$, Abramowitz e Stegun (1970) propõem as seguintes aproximações polinomiais para a avaliação de $I_{0}$ e $I_{1}$ :

$0 \leq x \leq 3,75$ :

$$
\begin{gathered}
I_{0}(x)=1+3,5156229 t^{2}+3,0899424 t^{4}+1,2067492 t^{6} \\
+0,2659732 t^{8}+0,0360768 t^{10}+0,0045813 t^{12}+\epsilon, \\
|\epsilon|<1,6 \times 10^{-7} \\
x^{-1} I_{1}(x)=0,5+0,87890594 t^{2}+0,51498869 t^{4}+0,15084934 t^{6} \\
+0,02658733 t^{8}+0,00301532 t^{10}+0,00032411 t^{12}+\epsilon ; \\
|\epsilon|<8,0 \times 10^{-9}
\end{gathered}
$$

$x \geq 3,75$ e $s=t^{-1}$ :

$$
\begin{aligned}
& x^{1 / 2} e^{-x} I_{0}(x)=0,39894228+0,01328592 s+0,00225319 s^{2} \\
&-0,00157565 s^{3}+0,00916281 s^{4}-0,02057706 s^{5} \\
&+0,02635537 s^{6}-0,01647633 s^{7}+0,00392377 s^{8}+\epsilon \\
&|\epsilon|<1,9 \times 10^{-7}
\end{aligned}
$$


e

$$
\begin{aligned}
& x^{1 / 2} e^{-x} I_{1}(x)=0,39894228-0,03988024 s-0,00362018 s^{2} \\
&+0,00163801 s^{3}-0,01031555 s^{4}+0,02282967 s^{5} \\
&-0,02895312 s^{6}+0,01787654 s^{7}-0,00420059 s^{8}+\epsilon . \\
&|\epsilon|<2,2 \times 10^{-7}
\end{aligned}
$$

\section{A.2.3 Avaliação da função inversa de $\mathcal{A}_{1}$}

A avaliação do valor da função inversa de $\mathcal{A}_{1}$ em um ponto $x>0$ pode ser feita através das seguintes aproximações:

$$
\operatorname{inv}\left\{\mathcal{A}_{1}(x)\right\}= \begin{cases}2 x+x^{3}+\frac{5 x}{6}, & \text { se } x<0,53 \\ -0,4+1,39 x+\frac{0,43}{1-x}, & \text { se } 0,53 \leq x<0,85 \mathrm{e} \\ \left\{x^{3}-4 x^{2}+3 x\right\}^{-1}, & \text { se } x \geq 0,85\end{cases}
$$




\section{Apêndice B}

\section{Resultados adicionais relativos a dados circulares}

Neste apêndice são apresentados alguns resultados relativos a dados circulares utilizados nesta tese.

\section{B.1 Intervalos de confiança}

São apresentadas abaixo as expressões dos intervalos de confiança para a média circular e parâmetro de concentração de uma amostra de tamanho $n$, independentes e identicamente distribuída de uma distribuição von Mises com média circular $\mu$ e parâmetro de concentração $\lambda$. Detalhes podem ser obtidos em Fisher (1993).

Sejam $\hat{\mu}_{n}$, dado por (1.1) e $\hat{\lambda}_{n}=\mathcal{A}^{-1}(\bar{R})$ os estimadores de máxima verossimilhança de $\mu$ e $\lambda$, respectivamente. O erro-padrão circular de $\hat{\mu}_{n}$ é definido por

$$
\hat{\sigma}_{V M}=\frac{1}{\left(n \bar{R} \hat{\lambda}_{n}\right)^{1 / 2}} .
$$

Um intervalo aproximado de $100(1-\alpha) \%$ de confiança para $\mu$ é dado por

$$
\left[\hat{\mu}-\operatorname{arcsen}\left(z_{\frac{1}{2} \alpha} \hat{\sigma}_{V M}\right) ; \hat{\mu}+\operatorname{arcsen}\left(z_{\frac{1}{2} \alpha} \hat{\sigma}_{V M}\right)\right] \text {, }
$$

onde $z_{\frac{1}{2} \alpha}$ é o $100(1-\alpha / 2) \%$ percentil da distribuição normal padrão.

Sejam

$$
a=\frac{n-R}{\chi_{n-1}^{2}\left(z_{1-\frac{1}{2} \alpha}\right)} \text { e } b=\frac{n-R}{\chi_{n-1}^{2}\left(z_{\frac{1}{2} \alpha}\right)},
$$


onde $\chi_{n-1}^{2}(c)$ corresponde ao $100(1-c) \%$ percentil da distribuição qui-quadrado com $n-1$ graus de liberdade. Um intervalo de $100(1-\alpha) \%$ de confiança para $\lambda$ é dado por

$$
\left[\frac{1+(1+3 a)^{1 / 2}}{4 a} ; \frac{1+(1+3 b)^{1 / 2}}{4 b}\right] .
$$

\section{B.2 Outros resultados}

Seja $y \sim \mathcal{M}(\mu ; \lambda)$; então

a. $E\left\{\operatorname{sen}^{2}(y-\mu)\right\}=\frac{\mathcal{A}_{1}(\lambda)}{\lambda} \mathrm{e}$

b. $E\left\{\cos ^{2}(y-\mu)\right\}=1-\frac{\mathcal{A}_{1}(\lambda)}{\lambda}$.

\section{Prova.}

a. Utilizando-se identidades trigonométricas, fórmulas de recorrência apresentadas na Seção A.2.1 e as expressões dos momentos trigonométricos da von Mises, apresentados na Seção 1.1.2, vem que

$$
E\left\{\operatorname{sen}^{2}(y-\mu)\right\}=\frac{1-E\{\cos 2(y-\mu)\}}{2}=\frac{\mathcal{A}_{1}(\lambda)}{\lambda} .
$$

b. Esse item pode ser provado utilizando-se a relação $\operatorname{sen}^{2} a+\cos ^{2} a=1$. 


\section{Apêndice C}

\section{Resultados adicionais}

Resultados relacionados a (3.27):

$$
\begin{gathered}
\mathbf{S}_{11 i}=\mathbf{D}_{i}^{* \top} \mathbf{W}_{i}^{-1} \mathbf{D}_{i}^{*}, \\
\mathbf{S}_{23 i}=\mathbf{S}_{32 i}^{\top}=\mathbf{Z}_{i}^{\top} \mathbf{F}_{i}\left[-\sigma^{2} E\left(\mathbf{p}_{2 i}\right)-\mathbf{A}_{i}^{-2} E\left\{\Delta^{-1}\left(\mathbf{a}_{i}\right) \dot{\mathbf{a}}_{i}\right\}+\frac{1}{2 \sigma^{4}} E\left(\mathbf{d}_{i}\right)\right], \\
\mathbf{p}_{2 i}=\mathbf{A}_{i}^{-3} \delta\left[\left\{\left(\ddot{\mathbf{a}}_{i}\right) \Delta\left(\mathbf{a}_{i}\right)-\Delta^{2}\left(\dot{\mathbf{a}}_{i}\right)\right\} \Delta^{-2}\left(\mathbf{a}_{i}\right)\right], \\
\mathbf{S}_{22 i}=\sigma^{2} \mathbf{Z}_{i}^{\top} \mathbf{F}_{i} \mathbf{A}_{i}^{-1}\left\{\sigma^{2} E\left(\mathbf{p}_{2 i}\right)+2 E\left(\mathbf{p}_{1 i}\right)\right\} \mathbf{F}_{i} \mathbf{Z}_{i}, \\
\mathbf{S}_{33 i}=\sum_{j=1}^{t}\left[\frac{1}{w_{i j}^{2}} E\left(\frac{\ddot{a}_{i j} a_{i j}-\dot{a}_{i j}^{2}}{a_{i j}^{2}}\right)-\frac{w_{i j}}{\sigma^{6}} E\left\{d\left(y_{i j} ; \mu_{i j}\right)\right\}\right] \\
\mathbf{V}_{11 i}=\mathbf{D}_{i}^{* \top} \mathbf{W}_{i}^{-1} \operatorname{Cov}\left(\mathbf{u}_{i}\right) \mathbf{W}_{i}^{-1} \mathbf{D}_{i}^{*}, \\
\mathbf{V}_{12 i}=\mathbf{V}_{21 i}^{\top}=\mathbf{D}_{i}^{* \top} \mathbf{W}_{i}^{-1} E\left\{\mathbf{u}_{i}\left(\sigma^{2} \mathbf{p}_{i 1}+\frac{1}{2 \sigma^{2}} \mathbf{d}_{i}\right)^{\top}\right\} \mathbf{F}_{i} \mathbf{Z}_{i}, \\
\mathbf{V}_{13 i}=\mathbf{V}_{31 i}=\mathbf{D}_{i}^{* \top} \mathbf{W}_{i}^{-1} E\left(\mathbf{u}_{i} s_{i}\right), \\
\mathbf{V}_{22 i}=\mathbf{Z}_{i}^{\top} \mathbf{F}_{i} E\left\{\left(\sigma^{2} \mathbf{p}_{1 i}+\frac{1}{2 \sigma^{2}} \mathbf{d}_{i}\right)\left(\sigma^{2} \mathbf{p}_{1 i}+\frac{1}{2 \sigma^{2}} \mathbf{d}_{i}\right)^{\top}\right\} \mathbf{F}_{i} \mathbf{Z}_{i}, \\
\mathbf{V}_{23 i}=\mathbf{V}_{32 i}^{\top}=\mathbf{Z}_{i}^{\top} \mathbf{F}_{i} E\left\{\left(\sigma^{2} \mathbf{p}_{2 i}^{-2}+\frac{1}{2 \sigma^{2}} \mathbf{d}_{i}\right) s_{i}\right\} \\
\mathbf{V}_{33 i}=E\left(s_{i}^{2}\right) .
\end{gathered}
$$




\section{Apêndice $\mathbb{D}$}

\section{Avaliação de erros para o cálculo da densidade da normal arqueada}

Este apêndice visa complementar a Seção 4.1 no que se refere à avaliação de séries necessárias ao cálculo da densidade da normal arqueada multivariada. Será utilizada a mesma notação daquela seção.

\section{D.1 Avaliação das séries via truncamento}

Seja $\left\{\mathcal{R}_{1}, \mathcal{R}_{2}\right\}$ uma partição de $\mathcal{Z}^{t}$, tal que $\mathcal{R}_{1}$ seja um conjunto finito e considere que as séries sejam aproximadas pela soma dos termos $\mathbf{k}_{i}$ tal que $\mathrm{k}_{i} \in \mathcal{R}_{1}$.

\section{Avaliação dos erros}

Inicialmente considere a série $e_{i}$. Reescreva-a como

$$
e_{i}=\sum_{\mathbf{k}_{i} \in \mathcal{R}_{1}} e_{i \mathbf{k}}+\sum_{\mathbf{k}_{i} \in \mathcal{R}_{2}} e_{i \mathbf{k}} .
$$

O erro na avaliação da série é

$$
\sum_{\mathbf{k}_{i} \in \mathcal{R}_{2}} e_{i \mathbf{k}}=e_{i}-\sum_{\mathbf{k}_{i} \in \mathcal{R}_{1}} e_{i \mathbf{k}}
$$


Contudo

$$
\begin{aligned}
\sum_{\mathbf{k}_{i} \in \mathcal{R}_{2}} e_{i \mathbf{k}} & \leq \int_{\mathbf{z} \in \mathcal{R}_{2}^{*}} \exp \left\{-\frac{1}{2}\left(\mathbf{y}_{i}+2 \mathbf{z}_{i} \pi-\boldsymbol{\mu}_{i}\right)^{\top} \boldsymbol{\Sigma}^{-1}\left(\mathbf{y}_{i}+2 \mathbf{z}_{i} \pi-\boldsymbol{\mu}_{i}\right)\right\} \\
& =(2 \pi)^{t / 2}|\boldsymbol{\Sigma}|^{1 / 2} P\left(\mathbf{Z}_{i} \in \mathcal{R}_{2}^{*}\right)
\end{aligned}
$$

onde $\mathbf{Z}_{i} \sim \mathcal{N}\left(\mathbf{y}_{i}-\boldsymbol{\mu}_{i} ; \boldsymbol{\Sigma}\right)$ e $\mathcal{R}_{2}^{*}$ é um subconjunto de $\Re^{t}$, tal que $\mathcal{R}_{2} \subset \mathcal{R}_{2}^{*}$, que não contém $\mathcal{R}_{1}$ e tal que a desigualdade acima seja válida. Logo o erro de aproximação é diretamente proporcinal a uma probabilidade de caudas de uma distribuição normal multivariada.

Analogamente, o erro de avaliação da série $\sum_{\mathbf{k}_{i} \in \mathcal{Z}^{t}} \mathbf{k}_{i} e_{i \mathbf{k}}$ é

$$
\begin{aligned}
\sum_{\mathbf{k}_{i} \in \mathcal{R}_{2}} \mathbf{k}_{i} e_{i \mathbf{k}} & \leq \int_{\mathbf{z}_{i} \in \mathcal{R}_{2}^{*}} \mathbf{z}_{i} \exp \left\{-\frac{1}{2}\left(\mathbf{y}_{i}+2 \mathbf{z}_{i} \pi-\boldsymbol{\mu}_{i}\right)^{\top} \boldsymbol{\Sigma}^{-1}\left(\mathbf{y}_{i}+2 \mathbf{z}_{i} \pi-\boldsymbol{\mu}_{i}\right)\right\} \\
& =(2 \pi)^{t / 2}|\boldsymbol{\Sigma}|^{1,2} E\left(\mathbf{Z}_{i} \mathbf{1}_{\mathcal{R}_{2}^{*}}\right),
\end{aligned}
$$

onde $\mathbf{1}_{\mathcal{R}_{2}^{*}}$ é a função indicadora do conjunto $\mathcal{R}_{2}^{*}$.

Do mesmo modo, o erro de avaliação para $\sum_{\mathbf{k}_{i} \in \mathcal{Z}^{t}} \mathbf{k}_{i} \mathbf{k}_{i}^{\top} e_{i \mathbf{k}}$ é menor ou igual a $(2 \pi)^{t / 2}|\Sigma|^{1 / 2} E\left(\mathbf{Z}_{i} \mathbf{Z}_{i}^{\top} \mathbf{1}_{\mathcal{R}_{2}^{*}}\right)$.

Há várias possibilidades para a escolha da partição e dentre elas destacamos:

i. $\mathcal{R}_{2}=\left\{\mathrm{k}_{i} \in \mathcal{Z}^{t}:\left\|\mathrm{k}_{i}\right\|>l_{i}\right\}$, onde $l_{i}$ é um número real positivo;

ii. seja $l_{i} \in \mathcal{Z}^{t}$ e defina $\mathbf{k}_{i}>\mathbf{l}_{i}$ como indicador de $k_{i j}>l_{i j}, j=1, \ldots, t$ : $\mathcal{R}_{2}=\left\{\mathbf{k}_{i} \in \mathcal{Z}^{t}: \mathbf{k}_{i}>\mathrm{l}_{i}\right\}$

iii. $\mathcal{R}_{2}=\left\{\mathbf{k}_{i} \in \mathcal{Z}^{t}:\left(\mathbf{y}_{i}-\boldsymbol{\mu}_{i}-\mathbf{k}_{i}\right)^{\top} \boldsymbol{\Sigma}^{-1}\left(\mathbf{y}_{i}-\boldsymbol{\mu}_{i}-\mathbf{k}_{i}\right)>l_{i}\right\}$. A vantagem dessa partição é que

$$
P\left(\mathbf{Z}_{i} \in \mathcal{R}_{2}^{\bullet}\right)=\xi_{t ; l_{i}^{\bullet}}
$$

onde $\mathcal{R}_{2}^{\bullet}$ é um subconjunto de $\Re^{t}$, tal que $\mathcal{R}_{2} \subset \mathcal{R}_{2}^{\bullet}$, que não contém $\mathcal{R}_{1}$ e tal que a desigualdade acima seja válida; $\xi_{t ; l_{i}}$ indica a probabilidade de uma distribuição qui-quadrado com $t$ graus de liberdade assumir valores maiores ou iguais a $l_{i}^{\bullet}$. 


\section{Avaliação das séries via método de Monte Carlo}

Seja $\mathbf{Q}_{i} \sim \mathcal{N}_{t}\left(\mathbf{0} ; \mathbf{I}_{t}\right)$ e defina o vetor aleatório $\mathbf{L}_{i}=\left[\mathbf{Q}_{i}\right]$, cujos componentes correspondem ao maior inteiro contido em cada componente de $\mathbf{Q}_{i}$. Consequentemente,

$$
P\left(\mathbf{L}_{i}=\mathbf{k}_{i}\right)=\prod_{j=1}^{t} P\left(k_{i j} \leq \mathbf{L}_{i j} \leq k_{i j}+1\right)=P\left(\mathbf{k}_{i}\right) .
$$

Defina a variável aleatória

$$
A_{i}=\frac{e_{i \mathbf{L}_{i}}}{P\left(\mathbf{L}_{i}\right)}
$$

Tem-se que

$$
E\left(A_{i}\right)=\sum_{\mathbf{k}_{i} \in \mathcal{Z}^{t}} \frac{e_{i \mathbf{k}}}{P\left(\mathbf{k}_{i}\right)} P\left(\mathbf{k}_{i}\right)=\sum_{\mathbf{k}_{i} \in \mathcal{Z}^{\mathfrak{t}}} \epsilon_{i \mathbf{k}}=e_{i}
$$

Desse modo, um procedimento para a avaliação de $e_{i}$ consiste em gerar um grande número de realizações independentes, diga $m_{1}$, da variável aleatćria $A_{i}$ e estimar $e_{i}$ por

$$
\bar{A}_{i}=\sum_{j=1}^{m} \frac{e_{i \mathbf{k}_{i j}}}{P\left(\mathbf{k}_{i j}\right)} \frac{1}{m_{1}} .
$$

A lei forte dos grandes números garante que $\bar{A}_{i}$ converge quase-certamente para $e_{i}$ conforme $m$ cresce.

Analogamente, pode-se avaliar $\sum_{\mathbf{k}_{i} \in \mathcal{Z}^{t}} \mathbf{k}_{i} e_{i \mathbf{k}}$ através da geração de $m_{2}$ realizações independentes da variável aleatória

$$
\mathbf{B}_{i}=\mathbf{L}_{i} \frac{e_{i \mathbf{k}}}{P\left(\mathbf{k}_{i}\right) \bar{A}_{i}}
$$

Esse vetor aleatório tem esperança $\sum_{\mathbf{k}_{i} \in \mathcal{Z}^{t}} \mathbf{k}_{i} e_{i \mathbf{k}}$. A média amostral das observações geradas converge, pela lei forte dos grandes números, quase-certamente para $\sum_{\mathbf{k}_{i} \in \mathcal{Z}^{t}} \mathrm{k}_{i} e_{i \mathbf{k}}$.

Por fim, o mesmo tipo de resultado pode ser obtido para a avaliação de $\sum_{\mathbf{k}_{i} \in \mathcal{Z}^{t}} e_{i \mathbf{k}} \mathbf{k}_{i} \mathbf{k}_{i}^{\top}$, através da média aritmética de um alto número de valores independentemente gerados de uma matriz aleatória

$$
\mathbf{C}_{i}=e_{i \mathrm{k}} \frac{\mathbf{L}_{i} \mathbf{L}_{i}^{\top}}{P\left(\mathbf{L}_{i}\right)}
$$




\section{Apêndice E}

\section{Complemento da aplicação}

Neste apêndice são apresentadas as estimativas preliminares das matrizes de correlação envolvidas na aplicação. $\hat{\mathbf{W}}_{i}$ e $\hat{\mathbf{W}}_{i}^{o}$ correpondem às matrizes de correlação entre senos e cossenos dos ângulos centrados na média circular, repectivamente.

Local: CMC-I, período de soltura: matutino

$$
\hat{\mathbf{W}}_{i}=\left(\begin{array}{lll}
1,00 & 0,81 & 0,63 \\
0,81 & 1,00 & 0,83 \\
0,63 & 0,83 & 1,00
\end{array}\right) \quad \hat{\mathbf{W}}_{i}^{0}=\left(\begin{array}{lll}
1,00 & 0,85 & 0,38 \\
0,85 & 1,00 & 0,64 \\
0,38 & 0,64 & 1,00
\end{array}\right)
$$

Local: CMC-I, período de soltura: meio-dia

$$
\hat{\mathbf{W}}_{i}=\left(\begin{array}{ccc}
1,00 & 0,85 & 0,78 \\
0,85 & 1,00 & 0,90 \\
0,78 & 0,90 & 1,00
\end{array}\right) \quad \hat{\mathbf{W}}_{i}^{0}=\left(\begin{array}{lll}
1,00 & 0,84 & 0,80 \\
0,85 & 1,00 & 0,94 \\
0,80 & 0,94 & 1,00
\end{array}\right)
$$

Local: CMC-I, período de soltura: tarde

$$
\hat{\mathbf{W}}_{i}=\left(\begin{array}{lll}
1,00 & 0,70 & 0,56 \\
0,70 & 1,00 & 0,60 \\
0,56 & 0,60 & 1,00
\end{array}\right) \quad \hat{\mathbf{W}}_{i}^{0}=\left(\begin{array}{lll}
1,00 & 0,70 & 0,06 \\
0,70 & 1,00 & 0,50 \\
0,06 & 0,50 & 1,00
\end{array}\right)
$$

Local: CMC-II, periodo de soltura: matutino 


$$
\hat{\mathbf{W}}_{i}=\left(\begin{array}{lll}
1,00 & 0,67 & 0,69 \\
0,67 & 1,00 & 0,93 \\
0,69 & 0,93 & 1,00
\end{array}\right) \quad \hat{\mathbf{W}}_{i}^{0}=\left(\begin{array}{lll}
1,00 & 0,19 & 0,22 \\
0,19 & 1,00 & 0,66 \\
0,22 & 0,66 & 1,00
\end{array}\right)
$$

Local: CMC-II, período de soltura: meio-dia

$$
\hat{\mathbf{W}}_{i}=\left(\begin{array}{lll}
1,00 & 0,38 & 0,15 \\
0,38 & 1,00 & 0,57 \\
0,15 & 0,57 & 1,00
\end{array}\right) \quad \hat{\mathbf{W}}_{i}^{0}=\left(\begin{array}{lll}
1,00 & 0,88 & 0,60 \\
0,88 & 1,00 & 0,79 \\
0,60 & 0,79 & 1,00
\end{array}\right) .
$$

Local: CMC-II, periodo de soltura: tarde

$$
\hat{\mathbf{W}}_{i}=\left(\begin{array}{lll}
1,00 & 0,58 & 0,40 \\
0,58 & 1,00 & 0,82 \\
0,40 & 0,82 & 1,00
\end{array}\right) \quad \hat{\mathbf{W}}_{i}^{0}=\left(\begin{array}{lll}
1,00 & 0,19 & 0,30 \\
0,19 & 1,00 & 0,61 \\
0,30 & 0,61 & 1,00
\end{array}\right)
$$




\section{Apêndice F}

\section{Resultados relativos a cópulas}

Nesse apêndice demonstramos alguns resultados sobre cópulas mencionados na Seção 6.2.

Proposição 4 Seja $\mathbf{y}^{\top}=\left(y_{1}, \ldots, y_{t}\right)$ um vetor aleatório absolutamente contínuo com função distribuição $G$, e seja $G_{i}$ a função distribuição de $y_{i}, i=$ $1,2, \ldots, t$. Então

$$
C(\mathbf{u})=G\left\{G_{1}^{-1}\left(u_{1}\right), G_{2}^{-1}\left(u_{2}\right), \ldots, G_{t}^{-1}\left(u_{t}\right)\right\},
$$

com $0<u_{i}<1$, é uma cópula.

\section{Prova:}

$$
\begin{aligned}
C(\mathbf{u}) & =P\left\{y_{1} \leq G_{1}^{-1}\left(u_{1}\right), y_{2} \leq G_{2}^{-1}\left(u_{2}\right), \ldots, y_{t} \leq G_{t}^{-1}\left(u_{t}\right)\right\} \\
& =P\left\{F_{1}\left(y_{1}\right) \leq u_{1}, F_{2}\left(y_{2}\right) \leq u_{2}, \ldots, F_{t}\left(y_{t}\right) \leq u_{t}\right\} \\
& =P\left(U_{1} \leq u_{1}, U_{2} \leq u_{2}, \ldots, U_{t} \leq u_{t}\right),
\end{aligned}
$$

onde $U_{i}$ segue uma distribuição uniforme no intervalo $(0,1)$. o

Proposição 5 Seja $C(\mathbf{u})$ uma cópula. Se $Y_{i}$ é uma variável aleatória absolutamente contínua com função distribuição $F_{i}, i=1,2, \ldots, t$, então

$$
F(\mathrm{y})=C\left\{F_{1}\left(y_{1}\right), F_{2}\left(y_{2}\right), \ldots, F_{t}\left(y_{t}\right)\right\},
$$

é uma função distribuição acumulada multivariada com marginais $F_{i}, i=$ $1,2, \ldots, t$. 
Prova: Seja $\mathbf{U}=\left(U_{1}, U_{2}, \ldots, U_{t}\right)$ o vetor aleatório correspondente à cópula $C$. Então

$$
\begin{aligned}
F(\mathrm{y}) & =P\left\{U_{1} \leq F_{1}\left(y_{1}\right), U_{2} \leq F_{2}\left(y_{2}\right), \ldots, U_{t} \leq F_{t}\left(y_{t}\right)\right\} \\
& =P\left\{F_{1}^{-1}\left(U_{1}\right) \leq y_{1}, F_{2}^{-1}\left(U_{2}\right) \leq y_{2}, \ldots, F_{t}^{-1}\left(U_{t}\right) \leq y_{t}\right\} \\
& =P\left(Y_{1} \leq y_{1}, Y_{2} \leq y_{2}, \ldots, Y_{t} \leq y_{t}\right) . \circ
\end{aligned}
$$

